\title{
Intrinsic Geometry of Varifolds in Riemannian Manifolds: Monotonicity and Poincare-Sobolev Inequalities
}

\author{
Julio Cesar Correa Hoyos
}

\author{
TESE APRESENTADA
}

$\mathrm{AO}$

Instituto De Matemática e Estatística

DA

Universidade DE SÃo PAUlo

PARA

OBTENÇÃO DO TÍTULO

$\mathrm{DE}$

DOUTOR EM CIÊNCIAS

Programa: Doutorado em Matemática

Orientador: Prof. Dr. Stefano Nardulli

Durante o desenvolvimento deste trabalho o autor recebeu auxílio financeiro da CAPES e CNPq

São Paulo, Agosto de 2020 
Intrinsic Geometry of Varifolds in Riemannian Manifolds:

Monotonicity and Poincare-Sobolev Inequalities

Esta é a versão original da tese elaborada pelo candidato Julio Cesar Correa Hoyos, tal como submetida à Comissão Julgadora. 


\section{Resumo}

CORREA HOYOS, J.C. Geometría Intrínsica de Varifolds em Variedades Riemannianas: Monotonia e Desigualdades do Tipo Poincaré-Sobolev . 2010. 120 f. Tese (Doutorado) Instituto de Matemática e Estatística, Universidade de São Paulo, São Paulo, 2020.

São provadas desigualdades do tipo Poincaré e Sobolev para funções com suporte compacto definidas em uma varifold $k$-rectificavel $V$ definida em uma variedade Riemanniana com raio de injetividade positivo e curvatura secional limitada por cima. As técnica usadas permitem considerar variedades Riemannianas $\left(M^{n}, g\right)$ com métrica $g$ de classe $C^{2}$ ou mais regular, evitando o uso do mergulho isométrico de Nash. Dito análise permite re-fazer algums fragmentos importantes da teoría geométrica da medida no caso das variedades Riemannianas com métrica $C^{2}$, que não é $C^{k+\alpha}$, com $k+\alpha>2$. A classe de varifolds consideradas, são aquelas em que sua primeira variação $\delta V$ esta em um espaço de Labesgue $L^{p}$ com respeito à sua medida de massa $\|V\|$ com expoente $p \in \mathbb{R}$ satisfazendo $p>k$.

Palavras-chave: Geometría métrica, cálculo das variações, teoría geomética da medida, análise em variedades, primeira variação de uma varifold, desigualdade de Michael-Simon. 


\section{Abstract}

CORREA HOYOS, J. C. Intrinsic Geometry of Varifolds in Riemannian Manifolds: Monotonicity and Poincare-Sobolev Inequalities. 2010. 120 f. Tese (Doutorado) - Instituto de Matemática e Estatística, Universidade de São Paulo, São Paulo, 2020.

We prove a Poincaré, and a general Sobolev type inequalities for functions with compact support defined on a $k$-rectifiable varifold $V$ defined on a complete Riemannian manifold with positive injectivity radius and sectional curvature bounded above. Our techniques allow us to consider Riemannian manifolds $\left(M^{n}, g\right)$ with $g$ of class $C^{2}$ or more regular, avoiding the use of Nash's isometric embedding theorem. Our analysis permits to do some quite important fragments of geometric measure theory also for those Riemannian manifolds carrying a $C^{2}$ metric $g$, that is not $C^{k+\alpha}$ with $k+\alpha>2$. The class of varifolds we consider are those which first variation $\delta V$ lies in an appropriate Lebesgue space $L^{p}$ with respect to its weight measure $\|V\|$ with the exponent $p \in \mathbb{R}$ satisfying $p>k$.

Keywords: Metric geometry, calculus of variations, geometric measure theory, analysis on manifolds, first variation of a varifold, Michael-Simon inequality. 


\section{Contents}

1 Introduction $\quad 1$

1.1 A quick overview . . . . . . . . . . . . . . . . . . 2

1.2 Monotonicity Inequality . . . . . . . . . . . . . . . . . . . . 3

1.3 Rectifiable varifolds . . . . . . . . . . . . . . . . . . . . . . 4

1.4 Poincaré and Sobolev inequalities . . . . . . . . . . . . . . . . . . 4

1.5 Structure of this thesis $\ldots \ldots \ldots \ldots \ldots \ldots \ldots$

2 Preliminaries $\quad 7$

2.1 Basic Geometric Measure Theory . . . . . . . . . . . . . . . . . . . . . 7

2.1 .1 Radon Measures . . . . . . . . . . . . . . . . . . . 7

2.1 .2 Convergence of Radon measures . . . . . . . . . . . . . . . . . . . 8

2.1 .3 Covering Theorems . . . . . . . . . . . . . . . . . . . . . . 9

2.1.4 Densities and Differentiation of measures . . . . . . . . . . . . . . . . 12

2.1.5 Grassmanian manifold . . . . . . . . . . . . . . . . . . . . 13

2.2 Riemannian Geometry . . . . . . . . . . . . . . . . . . . . . . 14

2.2 .1 Lie Derivatives and the Metric . . . . . . . . . . . . . . . 17

2.2 .2 Jacobi Fields and Rauch Comparison Theorem . . . . . . . . . . . . . . . 18

2.2 .3 Geometric Applications of Jacobi Fields Estimates . . . . . . . . . . . . . 20

3 Intrinsic Riemannian Theory of General Varifolds $\quad 25$

3.1 General Varifolds . . . . . . . . . . . . . . . . . . . . . . . 25

3.2 The First Variation of a Varifold . . . . . . . . . . . . . . . . . . 26

4 Monotonicity and Consequences $\quad 31$

4.1 Weighted Monotonicity Formulae for Abstrac Varifolds . . . . . . . . . . . . . 31

$4.2 \quad L^{p}$ Monotonicity Formula . . . . . . . . . . . . . . . . . . 38

5 Poincaré and Sobolev Type Inequalities for Intrinsic Varifolds 45

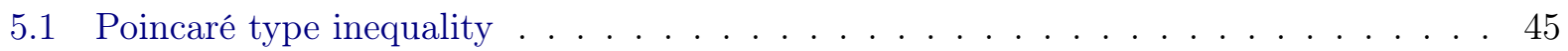

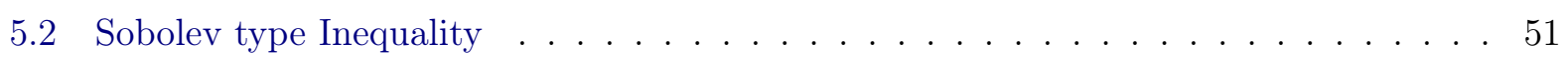

$\begin{array}{ll}\text { Bibliography } & 57\end{array}$ 
viii CONTENTS 


\section{Chapter 1}

\section{Introduction}

In the theory of PDE's the Sobolev spaces is the setting in which to apply ideas of functional analysis to glean information regarding various specific partial differential equations, in order to recast them abstractly as operators acting on appropriate linear spaces, where the operator encodes the structure of the partial differential equations, including possibly boundary conditions, etc. The great advantage is that once a PDE problem has been appropriately interpreted in this way, the general and elegant principles of functional analysis to study the solvability of various equations involving an abstract operator encoding the properties of the problem. The really hard work is not so much the invocation of functional analysis, but rather finding the "right" spaces and the "right" abstract operators. Sobolev spaces are designed precisely to make all this work out properly, and so these are usually the proper choices for.

Once this kind of spaces are at hand, an important questions is: whether a function on a Sobolev space belongs to a certain space? The answer depends on the appropriate choice of certain parameters as dimension and desired summability, and discover embeddings of various Sobolev spaces into others involves the uses of crucial analytic tools so-called "Sobolev type inequalities".

Among this "Sobolev type inequalities" there is the Gagliardo-Nirenberg-Sobolev inequality, which let the geometry enter at scene, relating them with the broadly studied isoperimetric inequality for domains in the euclidean space as follows.

Theorem 1.0.1 (c.f. [Eva10] sec. 5.6.1.). Assume $2 \leq p<n$. There exists a constant $C$, depending only on $p$ and $n$, such that

$$
\|u\|_{L^{p^{*}\left(\mathbb{R}^{n}\right)}} \leq C\|\nabla u\|_{L^{p}\left(\mathbb{R}^{n}\right)},
$$

for all $u \in C_{c}^{1}\left(\mathbb{R}^{n}\right)$, where $p^{*}=\frac{n p}{n-p}$.

In the special case in which $p=1$, and $u=\chi_{\Omega}$ (the characteristic function of $\Omega$ ), with $\Omega \subset$ $\mathbb{R}^{n}$ open set with boundary of class $C^{1}$, we can proof via integration by parts and a standard regularisation argument that:

$$
\left(\mathcal{L}^{n}(\Omega)\right)^{\frac{n-1}{n}} \leq C \mathcal{L}^{n}(\partial \Omega),
$$

where $\mathcal{L}^{n}$ is the $n$-dimensional measure of $\mathbb{R}^{n}$.

So, the study of this kind of "analytic inequalities" is in fact related with important geometric concepts, in fact in 1960, in his seminal work, [FF60] Federer and Fleming proves that once we can establish an isoperimetric inequality, there exists an associated Sobolev type inequality.

This is why Sobolev inequality has been studied for many years in the Euclidean case, as well as in the Riemannian case, and its prominent role in the theory of partial differential equations and geometry is well known. In [Mir67] Miranda obtained a Sobolev inequality for functions defined on minimal graphs, The Mirandas proof follows from the Isoperimetric inequality proved in [FF60] for integer currents and a procedure introduced by De Giorgi in [BDGM69a]. Bombieri used a refined version of this new inequality, De Giorgi and Miranda (employing the isoperimetric inequality of [FF60]), to derive gradient bounds for solutions to the minimal surface equation (see [BDGM69b]). 
In [MS73], Michael and Simon prove a general Sobolev type inequality, which proof follows an argument which is, in some aspects, evocative of the potential theory. That inequality is obtained on what might be termed a generalized manifold and in particular, the classical Sobolev inequality, a Sobolev inequality on graphs of weak solutions to the mean curvature equation, and a Sobolev inequality on arbitrary $C^{2}$ submanifolds of $\mathbb{R}^{n}$ (of arbitrary co-dimension) are derived.

On the other hand, Allard in a pioneering work [All72] proved a Sobolev type inequality for non-negative, compact supported functions defined on a varifold $V$, whose first variation $\delta V$ lies in an appropriate Lebesgue space endowed with the measure $\|\delta V\|$, by generalization of the Isoperimetric inequality for integer currents in [FF60]. The proof of this inequality given by Allards follows from the monotonicity formula derived from the computation of the first variation of a varifold for a suitable perturbation of a radial vector field.

Following the ideas of Michael and Simon, Hoffman and Spruk proved in [HS74] a general Sobolev inequality for submanifolds $N$ of a Riemannian manifold $M$, satisfying geometric restrictions involving the volume of $M$, the sectional curvatures and the injectivity radius of $M$. Their proof is inspired in the Michael and Simon work, therefore, is an extrinsic perspective.

Since this general Sobolev inequality has been largely studied in different contexts from an extrinsic point of view, a natural question is whether or not this kind of inequality remains valid from an intrinsic point o view. For functions with compact support on a varifold $V$ whose first variation $\delta V$ lies in an appropriate Lebesgue space with respect to $\|\delta V\|$.

In this thesis, we show an intrinsic Riemannian analog to the Allard result, considering a $k$ dimensional varifold $V$ defined in an $n$-dimensional Riemannian manifold $\left(M^{n}, g\right)$ (with $1 \leq k \leq$ $n$ ) defined intrinsically. We achieve this goal by recovering a monotonicity inequality (instead of monotonicity equality) in this intrinsic Riemannian context, which takes into account the bounds on the geometry of $M$, this procedure envolves ideas of the classical geometric measure theory and analysis on Riemannian manifolds. Then we follow the ideas of Simon and Michael in [MS73] and [Sim83], to get a local version of the desired inequality. Finally, The Sobolev type inequality is then obtained by a standard covering argument.

\subsection{A quick overview}

We present a natural extension of Allard's work. In fact, instead of define a general varifold on a Riemannian manifold via an isometric embedding (i.e., as a Radon measure on $G_{k}(i(M))$ where $i: M^{n} \rightarrow U \subset \mathbb{R}^{N}$ is an isometric embedding), is defined as a nonnegative, real extended valued, Radon measure on $G_{k}\left(M^{n}\right)$, the Grassmannian manifold whose underlying set is the union of the sets of $k$-dimensional subspaces of $T_{x} M$ as $x$ varies on $M^{n}$. This point of view has a consequence: more freedom on the regularity of the metric (i.e., our theory holds even for the case of only $C^{2}$ metrics, when the Nash embedding does not exists). In fact, there is a gap in the theory of isometric embeddings in $\mathbb{R}^{N}$, precisely in the case when the metric is $C^{2}$ but not $C^{2+\alpha}$ with $\alpha>0$ it is not known whether an isometric immersion into Euclidean space exists. On the other hand, whenever the metric is $C^{k, \alpha}$ with $k+\alpha>2$ there exists isometric embeddings of class $C^{k+\alpha}$. If the metric is $C^{k, \alpha}$ with $k+\alpha<2$ then there are isometric embeddings $C^{1+\frac{k+\alpha}{2}}$. The first theorem is proved with the aid of the "hard implicit function theorem", à la Nash-Moser. The second is proved using the technics of the first paper by John Nash about isometric embeddings, compare [Nas54]. This freedom constitutes, as shown in [Nar18] and [NOA18], a powerful tool to tackle problems in noncompact Riemannian manifolds of bounded geometry, or compact Riemannian manifolds with variable metric and also, possibly noncompact Riemannian manifolds of bounded geometry and variable metrics.

Our main goal is to reproduce Allard's Theorem 7.3 in this new context along the lines of the proofs of Theorems 18.5 and 18.6 of [Sim83]. To do so, as in [HS74], our ambient manifold must satisfy some geometric conditions, namely positive injectivity radius and sectional curvature 
bounded above, which from now on we refer as bounded geometry, see Definition 4.1.1. On the other hand, since we are interested in varifolds satisfying the conditions of the celebrated Theorem 8.1 [All72] (from now on called Allard Conditions $(A C)$, see Definition 3.2.3) is necessary to study the properties of varifolds satisfying these conditions. Roughly speaking, we are interested in varifolds whose first variation has no boundary term, having generalized mean curvature belonging to $L^{p}$ with respect to $\|\delta V\|$ for some $p>k$, and whose density ratio is close to that of an Euclidean disk in a small ball, and density bounded below far from $0,\|V\|$-a.e.

The main contributions of this paper are:

(i) to define rectifiable sets, exploiting the local structure of the ambient manifold, and so, define rectifiable varifolds as in Chapter 4 of [Sim83].

(ii) to give an intrinsic $L^{p}$-monotonicity formula valid for manifolds with bounded geometry and not only in $\mathbb{R}^{N}$, without using Nash's isometric embedding Theorem. See Theorem 1.

(iii) to prove, intrinsically, Poincaré and Sobolev type inequalities for $C^{1}(S)$ non-negative functions defined over a $\mathcal{H}_{g}^{k}$-rectifiable set $S \subset M$. Compare Theorems 2 and 3 .

(iv) to prove that the best constant $C>0$ in the extrinsic Sobolev inequality (1.4) depends only on the dimension $k$ of $V$, which is a remarkable fact, since one a priori expects that $C$ could depend also on the bounds of the geometry of the ambient manifold $n, k, i n j_{M}, b$. For the meaning of the former constants see Definition 4.1.1.

The rest of this introduction will describe in more detail the contributions of this paper.

\subsection{Monotonicity Inequality}

Given a general varifold $V$, the classic way to deduce a "monotonicity formula" is to compute the first variation of $V$ at a perturbations, by smooth functions, of (Euclidean) radial vector fields, then seems natural to consider vector fields of the form $\gamma\left(\frac{u}{s}\right)(u \nabla u)(x)$, where $\gamma(t)$ is a smooth real valued function which vanishes for large values of the variable $t$, and $u(x):=\operatorname{dist}_{(M, g)}(x, \xi)$ for some $\xi \in M$.

The classic calculation uses the fact that the divergence of a radial vector field over a $k$ dimensional plane is exactly $k$, which is not true in general Riemannian manifolds, so it is here where the bounded geometry plays a central role, since the Rauch comparison Theorem gives bounds on such quantity. The price to pay in making our monotonicity formula using this comparison geometry argument is that there is no equality anymore, instead we have the following inequality.

Theorem 1. Let $\left(M^{n}, g\right)$ be a complete Riemannian manifold with $g$ of class at least $C^{2}$ having bounded geometry and Levi-Civita connection $\nabla_{g}$, such that $r_{0} \cot _{b}\left(r_{0}\right)>0$, and let $V \in \mathbf{V}_{k}\left(M^{n}\right)$ satisfying $(A C)$, then for any $0<s<r_{0}$, and $h \in C^{1}(M)$ non-negative. There exists a constant $c=c(s, b) \in] 0,1\left[\right.$ such that, if we set $u(x)=r_{\xi}(x)=\operatorname{dist}_{(M, g)}(x, \xi)$ we have for all $0<s<r_{0}$

$$
\begin{aligned}
\frac{d}{d s}\left(\frac{1}{s^{k}} \int_{B_{g}(\xi, s)} h(y) d\|V\|(y)\right) & \geq \frac{d}{d s} \int_{G_{k}\left(B_{g}(\xi, s)\right)} h(y) \frac{\left|\nabla^{S^{\perp}} u\right|_{g}^{2}}{r_{\xi}^{k}} d V(y, T) \\
& +\frac{1}{s^{k+1}}\left(\int_{G_{k}\left(B_{g}(\xi, s)\right)}\langle\nabla h(y),(u \nabla u)(y)\rangle_{g} d V(y, T)\right) \\
& +\frac{(c(s)-1)}{s} \frac{k}{s^{k}} \int_{B_{g}(\xi, s)} h(y) d\|V\|(y) \\
& +\frac{1}{s^{k+1}} \int_{B_{g}(\xi, s)}\left\langle H_{g}, h(y)(u \nabla u)(y)\right\rangle_{g} d\|V\|(y) .
\end{aligned}
$$

Here $H_{g}$ is as in Proposition 3.2.1. 


\subsection{Rectifiable varifolds}

Here we define the main object of our investigation, namely rectifiable varifolds. We follow here the approach of Chapter 4 of [Sim83] without treating the rectifiability of general varifolds under conditions of the first variation and the density of their weights. Before to give the following definition it is worth to recall here the classical Rademacher Theorem.

Theorem 1.3.1 (Rademacher). If $f$ is Lipschitz on $\mathbb{R}^{n}$, then $f$ is differentiable $\mathcal{L}^{n}$-almost everywhere; that is, the gradient $\nabla f(x)$ exists and

$$
\lim _{y \rightarrow x} \frac{f(y)-f(x)-\nabla f(x) \cdot(y-x)}{|y-x|}=0,
$$

for $\mathcal{L}^{n}-a . e . x \in \mathbb{R}^{n}$

Definition 1.3.1 (cf. [AK00], Definition 5.3, pg. 536). Let $\left(M^{n}, g\right)$ be a complete Riemannian manifold. We say that a Borel set $S \subset M$ is countable $\mathcal{H}_{g}^{k}$-rectifiable if there exists countable many Lipschitz functions $f_{j}: \mathbb{R}^{k} \rightarrow M^{n}$ such that

$$
\mathcal{H}_{g}^{k}\left(S \backslash \bigcup_{j \in \mathbb{N}} f_{j}\left(\mathbb{R}^{k}\right)\right)=0 .
$$

For any $x \in \bigcup_{j \in \mathbb{N}} f_{j}\left(\mathbb{R}^{k}\right)$ such that $f_{j}$ is differentiable at $y=f_{j}^{-1}(x)$, we define the approximate tangent space $\operatorname{Tan}^{k}(S, x)$ as $d f_{y}\left(\mathbb{R}^{k}\right) \leq T_{y} M^{n}$.

Remark 1.3.1. Observe that the preceding definition is well posed, since, if $x \in f_{j}\left(\mathbb{R}^{k}\right) \cap f_{i}\left(\mathbb{R}^{k}\right)$ and $y_{l} \in f_{l}^{-1}(x)$ for $l \in\{i, j\}$, then $d f_{y_{i}}\left(\mathbb{R}^{k}\right)=d f_{y_{j}}\left(\mathbb{R}^{k}\right)$. The reader could consult [AK00], Definition 5.5, pg. 536 .

Definition 1.3.2 (c.f. [Sim83] Chapter 4, pg. 77). Let $S$ be a countably $\mathcal{H}_{g}^{k}$-rectifiable, subset of $\left(M^{n}, g\right)$ and let $\theta \in L_{l o c}^{1}\left(S, \mathcal{H}_{g}^{k}\right)$. Corresponding to such a pair $(S, \theta)$ we define the rectifiable $k$ varifold $\mathbf{v}(S, \theta)$ to be, the equivalence class of all pairs $(\tilde{S}, \tilde{\theta})$, where $\tilde{S}$ is countably $\mathcal{H}_{g}^{k}$-rectifiable with $\mathcal{H}_{g}^{k}((S \Delta \widetilde{S}))=0$ and where $\tilde{\theta}=\theta, \mathcal{H}_{g}^{k}-$ a.e. on $S \cap \tilde{S}$.

So, we can naturally induce a (general) varifold from a rectifiable one as follows.

Definition 1.3.3. Given an $\mathcal{H}_{g}^{k}$-rectifiable varifold $\mathbf{v}(S, \theta)$ on $M^{n}$ there is a corresponding (general) $k$-varifold $V \in \mathbf{V}_{k}\left(M^{n}\right)$ (also denoted by $\mathbf{v}(S, \theta)$ ), defined by

$$
V(A):=\mathbf{v}(S, \theta)(A)=\mathcal{H}_{g}^{k}\left\llcorner\theta\left(\pi\left(\left\{\left(x, T_{x} M\right): x \in S_{*}\right\} \cap A\right)\right), \quad A \subset G_{k}\left(M^{n}\right),\right.
$$

where $S_{*}$ is the set of $x \in S$ such that $S$ has an approximate tangent space $T_{x} S$ with respect to $\theta$ at $x$. Evidently $\mathbf{v}(S, \theta)$, so defined, has weight measure $\|\mathbf{v}(S, \theta)\|=\mathcal{H}_{g}^{k}\llcorner\theta$.

\subsection{Poincaré and Sobolev inequalities}

In the special case in which, $V=\mathbf{v}(S, \theta)$ is a rectifiable varifold such that the mean vector field belongs to a certain Lebesgue space $L^{p}$ with $p>k$, the Theorem 1 implies that, for $h \in C^{1}(S)$, $h \geq 0$,

$$
h(\xi) \leq e^{\left(\Lambda+c^{*} k\right) \rho}\left(\frac{1}{\omega_{k}} \rho^{k} \int_{B_{g}(\xi, \rho)} h d\|V\|+\int_{B_{g}(\xi, \rho)} \frac{\left|\nabla_{g}^{S} h\right|}{r_{\xi}^{k-1}} d\|V\|\right),
$$

for all $\xi \in \operatorname{spt}\|V\|$ and for all $0<\rho<r_{0}$. From this, together with an approximation argument and Fubini's Theorem we deduce the following Poincaré inequality. 
Theorem 2. Let $\left(M^{n}, g\right)$ be a complete Riemannian manifold with bounded geometry, let $V:=$ $\mathbf{v}(S, \theta)$ a rectifiable varifold satisfying $(A C)$. Suppose: $h \in C^{1}(M), h \geq 0, B_{g}(\xi, 2 \rho) \subset B_{g}\left(\xi, r_{0}\right)$ for $\xi \in S$ fixed, $\left|H_{g}\right|_{g} \leq \Lambda$ for some $\Lambda>0, \theta>1\|V\|$-a.e. in $B_{g}\left(\xi, r_{0}\right)$ and for some $0<\alpha<1$

$$
\|V\|\left(\left\{x \in B_{g}(\xi, \rho): h(x)>0\right\}\right) \leq \omega_{k}(1-\alpha) \rho^{k} \quad \text { and }, \quad e^{\left(\Lambda+c^{*} k\right) \rho} \leq 1+\alpha .
$$

Suppose also that, for some constant $\Gamma>0$

$$
\|V\|\left(B_{g}(\xi, 2 \rho)\right) \leq \Gamma \rho^{k} .
$$

Then there are constants $\left.\beta:=\beta\left(k, \alpha, r_{0}, b\right) \in\right] 0, \frac{1}{2}\left[\right.$ and $C:=C\left(k, \alpha, r_{0}, b\right)>0$ such that

$$
\int_{B_{g}(\xi, 2 \rho)} h d\|V\| \leq C \rho \int_{B_{g}(\xi, \rho)}\left|\nabla_{g}^{S} h\right|_{g} d\|V\| .
$$

Here $H_{g}$ is as in Proposition 3.2.1.

Finally, the Sobolev inequality follows from Theorem 1 again in the special case in which $V=$ $\mathbf{v}(S, \theta)$ is a rectifiable varifold such that the mean vector field belongs to a certain Lebesgue space and a standard covering argument (c.f. [Sim83] Theorem 3.3 pg. 11).

Theorem 3. Let $\left(M^{n}, g\right)$ be a complete Riemannian manifold with bounded geometry, let $V=$ $\mathbf{v}(S, \theta)$ a $k$-rectifiable varifold satisfying $(A C)$. Suppose $h \in C_{0}^{1}(M)$ non negative, and $\theta \geq 1\|V\|$ a.e. in $S$. Then there exists $C:=C(k)>0$ such that

$$
\left(\int_{S} h^{\frac{k}{k-1}} d\|V\|\right)^{\frac{k-1}{k}} \leq C \int_{S}\left(\left|\nabla_{g}^{S} h\right|_{g}+h\left(\left|H_{g}\right|_{g}-c^{*} k\right)\right) d\|V\| .
$$

Here $H_{g}$ is as in Proposition 3.2.1.

\subsection{Structure of this thesis}

In the Section 2 we make a review of the basic concepts of the classical geometric measure theory and Riemannian geometry which in the sequel will be used, the experienced reader may omit this section. In general in this section we give precise references for the classical results, and encourage the unaware reader to look them. In Section 3 the definition of general varifold of [Allz2] is extended to a complete Riemannian manifold as well as the first variation of them, extending the geometric theory of varifolds to this setting. In Section 4 Theorem 1 is proved, by testing the first variation of a general varifold with a suitable radial deformation field. Here the bounded geometry assumptions of the ambient manifold, play an essential role. In Section 4 are also proved several results concerning the monotonicity of the density ratio of the wight measure, under $L^{p}$-type assumptions on the weak mean curvature field $H_{g}$. Finally, in Section 5 Theorem 2 and Theorem 3 are proved, as a consequence of Theorem 1, standard covering argument, and several technical results from classical analysis. 


\section{Chapter 2}

\section{Preliminaries}

The aim of this chapter is to introduce the basic notations and classical results fo the Geometric Measure Theory and Riemannian Comparison Geometry, in an intrinsic setting, in order to cover all the basics, to tackle in Chapter 4, the intrinsic theory of general and rectifiable varifolds.

Since our goal is to obtain estimates on functions defined on a varifold which ambient space is a Riemnnian manifold, exploiting the geometry of the ambient space, and the monotonicity behaviour of the density of varifolds, the following results will be in the context of Riemannian manifold, or more generally in separabel metric spaces.

\subsection{Basic Geometric Measure Theory}

\subsubsection{Radon Measures}

Let us begin with the basics on Radon measures and their relation with continuous functions, those link between the topology of the set of continuous functions and the space of Radon measure is known as the Riesz representation theorem, and is the cornerstone of the geometric measure theory. The proof of the Theorem in this section, can be founded in [Mag12] Chapter 4 (from where was taken) or in [Sim83] Ch 1.

Definition 2.1.1. An outer measure $\mu$ is a Radon measure in $X$, if it is a locally finite (i.e. $\mu(K)<\infty$ for all $K \subset X$ compact), Borel regular measure on $X$.

If $\mu$ is a Radon measure, in particular is Borel regular, is well known that

$$
\mu(E)=\inf \{\mu(A): E \subset A, A \text { open set }\}=\sup \{\mu(K): K \subset E, K \text { compact set }\},
$$

for every $E \in \mathcal{B}(X)$. Thus by Borel regularity a Radon measure $\mu$ is characterized on $\mathcal{M}(\mu)$ by its behaviour on compact (or on open) sets.

It is clear that, if $\mu$ is a Borel regular measure on $X$, and $E \in \mathcal{M}(\mu)$ is such that $\mu(E)<\infty$, then $\mu\llcorner E$ is a Radon measure on $X$.

Definition 2.1.2. An outer measure $\mu$ on $\mathbb{R}^{n}$ is concentrated on $E \subset X$ if $\mu(X \backslash E)=0$. The intersection of the closed sets $E$ such that $\mu$ is concentrated on $E$ is denoted spt $\mu$ and called the support of $\mu$. Furthermore, if $X$ is metric, locally compact separable,

$$
X \backslash \operatorname{spt}(\mu)=\{x \in X: \mu(B(x, r))=0 \text { for some } r>0\} .
$$

Definition 2.1.3. Let $X$ be metric, locally compact, and separable, and $H$ a real Hilbert space, consider $L: C_{c}^{0}(X ; H) \rightarrow \mathbb{R}$ a linear functional, we define the total variation of $L$ as the set function

$$
|L|: \mathcal{P}(X) \rightarrow[0, \infty]
$$

such that, for any $A \subset X$ open set

$$
|L|(A):=\sup \left\{\langle L, \varphi\rangle: \varphi \in C_{c}^{0}(A ; H),|\varphi| \leq 1\right\},
$$


and for any arbitrary $E \subset X$

$$
|L|(E):=\inf \{|L|(A): E \subset A, A \text { open set }\} .
$$

Theorem 2.1.1 (Riez Representation Theorem). Let $X$ be a metric, locally compact and separable space, $H$ a real Hilbert space, if $L: C_{c}^{0}(X ; H) \rightarrow \mathbb{R}$ is a bounded linear functional (hence continuous), then its total variation $|L|$ is a positive Radon measure on $X$ and there exists a $|L|$-mesurable function $\nu: X \rightarrow H$, with $|\nu|=1,|L|$-a.e. on $X$ and

$$
\langle L, \varphi\rangle=\int_{X}\langle\varphi, \nu\rangle d|L|, \quad \text { for all } \varphi \in C_{c}^{0}(X, H),
$$

that is, $L=g|L|$. Moreover, for every open set $A \subset X$ we have

$$
|L|(A)=\sup \left\{\int_{X}\langle\varphi, g\rangle d|L|: \varphi \in C_{c}^{0}(A, H),|\varphi| \leq 1\right\},
$$

and for each arbitrary subset $E \subset X$ the following formula holds

$$
|L|(E):=\inf \{|L|(A): E \subset A \text { and } A \text { is open }\} .
$$

\subsubsection{Convergence of Radon measures}

Once establish the relation between radon measures and continuous functions, we begin to exploit the topology of this space to investigate compactness properties of the Radon measures, the proof in this section can be found in [Mag12] Chapter 4 and 5.

Let $X$ be a metric, locally compact and separable space, and $H$ a real Hilbert space, let $L$ be a monotone linear functional on $C_{c}^{0}(X, H)$, i.e., $L\left(\varphi_{1}\right) \leq L\left(\varphi_{2}\right)$ whenever $\varphi_{1} \leq \varphi_{2}$, then $L$ is bounded on $C_{c}^{0}(X, H)$, and by Theorem 2.1.1 it holds

$$
\langle L, \varphi\rangle=\int_{X}\langle\varphi, \nu\rangle d|L|,
$$

where $\nu: X \rightarrow H$ is $|L|$-mesurable, with $|\nu|=1|L|$-a.e. on $X$, Note also that if two Radon measures $\mu_{1}, \mu_{2}$ on $\mathbb{R}^{n}$ coincides as linear functionals, that is

$$
\int_{X} \varphi d \mu_{1}=\int_{\mathbb{R}^{n}} \varphi d \mu_{2}, \quad \forall \varphi \in C_{c}^{0}\left(\mathbb{R}^{n}\right),
$$

then we have $\mu_{1}=\mu_{2}$, so Radon measures can be unambiguously identified with monotone linear functionals on $C_{c}^{0}(X, H)$.

Now, let $\mathcal{B}_{b}(X)$ denote the family of bounded Borel sets of $X$ and $\mathcal{B}(E)$ the family of bounded sets contained in $E \subset X$. If $L$ is a bounded linear functional on $C_{c}^{0}\left(X ; \mathbb{R}^{n}\right)$ (we are considering the special case when $H=\mathbb{R}^{n}$ ), then $L$ induces a $\mathbb{R}^{n}$-valued set function

$$
\begin{aligned}
\nu: \mathcal{B}_{b}(X) & \rightarrow \mathbb{R} \\
E & \mapsto \nu(E):=\int_{E} \nu d|L|,
\end{aligned}
$$

that enjoys the $\sigma$-additivity property, i.e.

$$
\nu\left(\bigcup_{n \in \mathbb{N}} E_{n}\right)=\sum_{n \in \mathbb{N}} \nu\left(E_{n}\right),
$$

on every disjoint sequence $\left\{E_{n}\right\}_{n \in \mathbb{N}} \subset \mathcal{B}(K)$ for some $K$ compact set in $X$. 
Thus bounded linear functionals on $C_{c}^{0}\left(X ; \mathbb{R}^{n}\right)$ naturally induces a $\mathbb{R}^{m}$-valued set function on $\mathbb{R}^{n}$ that are $\sigma$-additive on bounded Borel sets, then is natural to define a notion of convergence on it.

Definition 2.1.4. Let $X$ as above, $\left\{\mu_{n}\right\}_{n \in \mathbb{N}}$ and $\mu$ Radon measures with values in $X$. We say that $\mu_{n}$ converge to $\mu$, and we write $\mu_{n} \stackrel{\star}{\rightarrow} \mu$, if

$$
\int_{X} \varphi d \mu=\lim _{n \rightarrow \infty} \int_{X} \varphi d \mu_{n}, \quad \text { for all } \varphi \in C_{c}^{0}\left(X ; \mathbb{R}^{n}\right)
$$

Notice that this notion of covergence coincides with the waek* convergence for the space $\left(C_{c}^{0}\left(X, \mathbb{R}^{m}\right)\right)$ /; and by the comments above we know that the space of Radon measures is contained in $\left(C_{c}^{0}\left(X, \mathbb{R}^{m}\right)\right)$ / this is the natural notion of convergence for Radon measures.

The following two Theorems, are important results concerning to weak* convergence of Radon measures and the compactness of this space, and will be used along the text repeatedly, the full proof of that can be found in [Sim83]

Proposition 2.1.1. Let $X$ a metric, locally compact and separable space. If $\left\{\mu_{n}\right\}_{n \in \mathbb{N}}$ and $\mu$ are Radon measures on $X$, then the following are equivalents

1. $\mu_{n} \stackrel{\star}{\rightarrow} \mu$.

2. If $K$ is a compact set and $A$ is an open set, then

$$
\begin{aligned}
& \mu(K) \geq \limsup _{n \rightarrow \infty} \mu_{n}(K), \\
& \mu(A) \leq \liminf _{n \rightarrow \infty} \mu_{n}(A) .
\end{aligned}
$$

3. If $E$ is a bounded Borel set with $\mu(\partial E)=0$, then

$$
\mu(E)=\lim _{n \rightarrow \infty} \mu_{n}(E) .
$$

Moreover if $\mu_{n} \stackrel{\star}{\rightarrow} \mu$ the for every $x \in \operatorname{spt} \mu$ there exists $\left\{x_{n}\right\}_{n \in \mathbb{N}} \subset X$ with

$$
\lim _{n \rightarrow \infty} x_{n}=x, x_{n} \in \text { Spt } \mu_{n}, \text { for all } n \in \mathbb{N} .
$$

Theorem 2.1.2 (Compactness Criteria for Radon Measures). Let $X$ a metric, locally compact and separable space. If $\left\{\mu_{n}\right\}_{n \in \mathbb{N}}$ is a sequence of Radon measures on $X$ such that, for all compact set $K$ in $X$

$$
\sup _{n \in \mathbb{N}} \mu_{n}(K)<\infty,
$$

then there exists a Radon measure $\mu$ on $X$ and a sequence $n(k) \rightarrow \infty$, as $k \rightarrow \infty$ such that

$$
\mu_{n(k)} \stackrel{\star}{\longrightarrow} \mu
$$

\subsubsection{Covering Theorems}

In this section we deal with some covering theorems, which will allow to deduce global properties of a Radon measure from local ones with respect to some subset.

Since the main goal is to deal with "measure theoretic" objects defined on a Riemannian manifold, we need a machinery which deals with the fact that we are not in an Euclidean space anymore to infer key properties of suitable Radon measures in the large. This theory was developed in more general context by H. Federer (c.f. [Fed69] Chapter 2).

From now, we will denote $(X, d)$ a locally compact and separable metric space, and a $\mu$ a Radon measure on $X$. we also denote $B_{d}(x, r):=\{y \in X: d(x, y)<r\}$ the open ball centered at $x \in X$ of radius $r>0$ on $X$ with respect to de distance $d$. 
Theorem 2.1.3. Let $\mathcal{B}=\left\{\bar{B}_{d}\left(x_{\alpha}, r_{\alpha}\right)\right\}_{\alpha \in \Lambda}$ any collection of closed balls in $X$, with

$$
\sup _{\alpha \in \Lambda}\left\{\operatorname{diam}\left(\bar{B}_{d}\left(x_{\alpha}, r_{\alpha}\right)\right)\right\}<\infty
$$

then, there exists a $\Lambda^{\prime} \subset \Lambda$ such that the subcollection $\mathcal{B}^{\prime}:=\left\{\bar{B}_{d}\left(x_{\beta}, r_{\beta}\right)\right\}_{\beta \in \Lambda^{\prime}} \subset \mathcal{B}$ satisfies:

$$
\bar{B}_{d}\left(x_{\beta}, r_{\beta}\right) \cap \bar{B}_{d}\left(x_{\eta}, r_{\eta}\right)=\emptyset \text { if } \beta, \eta \in \Lambda^{\prime} \text { with } \beta \neq \eta \text { and, } \bigcup_{\alpha \in \Lambda} \bar{B}_{d}\left(x_{\alpha}, r_{\alpha}\right) \subset \bigcup_{\beta \in \Lambda^{\prime}} \bar{B}_{d}\left(x_{\beta}, 5 r_{\beta}\right) .
$$

In fact we can arrange the following stronger property: if $\bar{B}_{d}\left(x_{\alpha}, r_{\alpha}\right) \in \mathcal{B}$ then there exists $\bar{B}_{d}\left(x_{\beta}, r_{\beta}\right) \in$ $\mathcal{B}^{\prime}$, such that $\bar{B}_{d}\left(x_{\alpha}, r_{\alpha}\right) \cap \bar{B}_{d}\left(x_{\beta}, r_{\beta}\right)=\emptyset$, and $\bar{B}_{d}\left(x_{\alpha}, r_{\alpha}\right) \subset \bar{B}_{d}\left(x_{\beta}, 5 r_{\beta}\right)$.

For a proof of this result the interested reader may see [Sim83] Theorem 1.3.3 pg. 11.

Nevertheless this result is very useful in presence of some "regularity hypothesis" of a Radon measure, and for most applications it is good enough to obtain global properties from local ones. However, deal with problems where no such regularity hypothesis is at hand, this forces the use of another type of covering theorems, which impose no special hypothesis on the measure, but require boundedness of the set of centers of a collection of closed balls and a geometric condition on the metric $d$, namely.

Definition 2.1.5. A set $A$ in a metric space $X$ is said to be directionally $(\delta, C)$ limited if for each $a \in A$ there exists at most $C$ distinct points $\xi_{1}, \ldots, \xi_{C}$ in $B_{d}(a, \delta) \cap A$ such that for $i \neq j$ and $x \in X$, the following holds: if

$$
d(a, x)+d\left(x, \xi_{i}\right)=d\left(a, \xi_{i}\right) \text { and } d\left(a, \xi_{j}\right)=d(a, x),
$$

then

$$
d\left(x, \xi_{j}\right) \geq \frac{1}{4} d\left(a, \xi_{j}\right) .
$$

The idea behind this definition occurs when we consider the special case in which $X=A=\mathbb{R}^{n}$, with the usual metric induced from the canonical norm $\|\cdot\|$. To see that $\mathbb{R}^{n}$ is directionally $(\delta, C(n))$ limited, for all $\delta>0$ and $C(n)$ a dimensional constant, assume $a=0$, and take $\xi_{i}, \xi_{j} \in \mathbb{R}^{n} \cap B_{d}(0, \delta)$ distinct such that $\left\|\xi_{i}\right\| \geq\left\|\xi_{j}\right\|$, then setting

$$
x=\frac{\xi_{i}\left\|\xi_{j}\right\|}{\left\|\xi_{i}\right\|}
$$

we have that

$$
\begin{aligned}
d\left(0, \xi_{j}\right) & =\left\|\xi_{j}\right\| \\
& =\left\|\frac{\xi_{j}\left\|\xi_{i}\right\|}{\left\|\xi_{i}\right\|}\right\| \\
& =\|x\|,
\end{aligned}
$$

and

$$
\begin{aligned}
d(0, x)+d\left(x, \xi_{i}\right) & =\|x\|+\left\|\frac{\xi_{i}\left\|\xi_{j}\right\|}{\left\|\xi_{i}\right\|}-\xi_{i}\right\| \\
& =\left\|\xi_{j}\right\|+\left\|\frac{\xi_{i}\left\|\xi_{j}\right\|}{\left\|\xi_{i}\right\|}-\xi_{i}\right\|
\end{aligned}
$$




$$
\begin{aligned}
& =\frac{\left\|\xi_{j}\right\|\left\|\xi_{i}\right\|+\left\|\xi_{i}\right\| \xi_{j}\left\|-\xi_{i}\right\| \xi_{i}\|\|}{\left\|\xi_{i}\right\|} \\
& =\frac{\left\|\xi_{j}\right\|\left\|\xi_{i}\right\|+\left\|\xi_{i}\right\|\left(\|\| \xi_{j}\|-\| \xi_{i}\|\|\right)}{\left\|\xi_{i}\right\|} \\
& =\frac{\left\|\xi_{i}\right\|}{\left\|\xi_{i}\right\|}\left(\left\|\xi_{j}\right\|+\|\| \xi_{j}\|-\| \xi_{i}\|\|\right) \\
& =\left\|\xi_{j}\right\|-\left\|\xi_{j}\right\|+\left\|\xi_{i}\right\| \\
& =d\left(0, \xi_{i}\right),
\end{aligned}
$$

where we use that $\left\|\xi_{i}\right\| \geq \| \xi_{j}$. Thus,

$$
\frac{d\left(x, \xi_{j}\right)}{d\left(0, \xi_{j}\right)}=\frac{1}{\left\|\xi_{i}\right\|}\left(\left\|\frac{\xi_{i}\left\|\xi_{j}\right\|}{\left\|\xi_{j}\right\|}-\xi_{j}\right\|\right)=\left\|\frac{\xi_{i}}{\left\|\xi_{i}\right\|}-\frac{\xi_{j}}{\left\|\xi_{j}\right\|}\right\| .
$$

On the other hand, since

$$
\mathbb{S}^{n}:=\left\{\xi \in \mathbb{R}^{n}: d(0, \xi)=1\right\},
$$

is compact, there exists $C(n)$ finite integer, and $\left\{U_{k}\right\}_{k=1}^{C(n)} \subset \mathbb{R}^{n}$ a collection of open sets such that $\mathbb{S}^{n} \subset \bigcup_{k=1}^{C(n)} U_{k}$ and $\operatorname{diam}\left(U_{k}\right) \geq \frac{1}{4}$, for all $k=1, \ldots, C(n)$. Then, there exists $i, j \in\{1, \ldots, C(n)\}$ such that $\frac{\xi_{i}}{\left\|\xi_{i}\right\|} \in U_{i} \cap \mathbb{S}^{n}$ and $\frac{\xi_{j}}{\left\|\xi_{j}\right\|} \in U_{j} \cap \mathbb{S}^{n}$, therefore, if we assume that neither $\frac{\xi_{i}}{\left\|\xi_{i}\right\|} \in U_{j}$ nor $\frac{\xi_{j}}{\left\|x_{j}\right\|} \in U_{i}$, we have that

$$
\frac{d\left(x, \xi_{j}\right)}{d\left(0, \xi_{j}\right)} \geq \frac{1}{4}
$$

then, there exists at most $C(n)$ different points fulfilling the conditions.

Finally, since $\delta$ is arbitrary, and without loss of generality the argument can be repeated for all $a \in \mathbb{R}^{n}$, follows that $\mathbb{R}^{n}$ is directionally $(\delta, C(n))$ limited.Thus, the word "directionaly" make sense, since there exists at most finite directions fulfiling the conditions.

Another interesting special case (whose study led to formulate the general concept) arises when $X=(M, g)$ is a complete Riemannian manifold with $g$ of class greater or equal than 2 and $A=$ $\bar{B}_{g}\left(\tilde{\xi}, r_{0}\right)$ is the geodesic closed ball centered at $\tilde{\xi} \in M$ and $r_{0}>0$ is such that

$$
\exp _{\tilde{\xi}}:\left\{v \in T_{\xi} M:\|v\| \leq r_{0}\right\} \subset T_{\xi} M \rightarrow \exp _{\tilde{\xi}}\left(\left\{v \in T_{\xi} M:|v| \leq r_{0}\right\}\right),
$$

is a diffeomorphism (by the completeness of $(M, g)$, such $r_{0}$ always exists and is positive). Since $\exp _{\tilde{\xi}}$ is a diffeomorphism, and $B_{g}\left(\tilde{\xi}, r_{0}\right)$ is a compact set, it is an bi-Lipschitz map with constant $\lambda>0$, i.e. for all $v_{i}, v_{j} \in T_{\tilde{\xi}} M$

$$
\frac{1}{\lambda} \delta\left(v_{i}, v_{j}\right) \leq g\left(v_{i}, v_{j}\right) \leq \lambda \delta\left(v_{i}, v_{j}\right)
$$

where $\delta(\cdot, \cdot)$ stands for the canonical metric on $\mathbb{R}^{n} \cong T_{\tilde{\xi}} M$. Hence, setting,

$$
\xi_{i}=\exp _{\tilde{\xi}}\left(v_{i}\right), \xi_{j}=\exp _{\tilde{\xi}}\left(v_{j}\right), \text { and } x=\exp _{\tilde{\xi}}\left(\frac{v_{i} \delta\left(v_{j}, v_{j}\right)}{\delta\left(v_{i}, v_{i}\right)}\right),
$$

where $r_{0} \geq\left\|v_{i}\right\| \geq\left\|v_{j}\right\|$, we have that,

$$
\frac{d_{g}\left(x, \xi_{j}\right)}{d_{g}\left(\tilde{\xi}, \xi_{j}\right)} \leq \lambda^{2} \frac{d_{\delta}\left(x, v_{j}\right)}{d_{\delta}\left(0, v_{j}\right)}=\left\|\frac{\xi_{i}}{\left\|\xi_{i}\right\|}-\frac{\xi_{j}}{\left\|\xi_{j}\right\|}\right\|
$$


where $d_{\delta}$ and $d_{g}$ are the distance induced respectively by $\delta$ and $g$. Then, reasoning as in the $\mathbb{R}^{n}$ case, we have that there exists $C(n, \lambda)$ positive integer and $0<\varepsilon(\tilde{\xi})<r_{0}$ such that, for $\xi_{i}, \xi_{j} \in B_{g}(\tilde{\xi}, \varepsilon(\tilde{\xi}))$ and $x$ as above, where $i, j \in\{1, \ldots, C(n)\}$ and if

$$
d_{g}(\tilde{\xi}, x)+d_{g}\left(x, \xi_{i}\right)=d_{g}\left(\tilde{\xi}, \xi_{i}\right) \text { and } d_{g}\left(\tilde{\xi}, \xi_{j}\right)=d(\tilde{\xi}, x),
$$

then

$$
d_{g}\left(x, \xi_{j}\right) \geq \frac{1}{4} d_{g}\left(\tilde{\xi}, \xi_{j}\right)
$$

Finally, by compactness of $\bar{B}_{g}\left(\tilde{\xi}, r_{0}\right), \varepsilon(\tilde{\xi})$ can be chosen uniform, satisfying that, for all $\xi \in B_{g}\left(\tilde{\xi}, r_{0}\right)$, the argument above apply in the smaller ball $B_{g}(\xi, \varepsilon)$ (this is possible by the compactness of the unit tangent vectors at the points of $\left.B_{g}\left(\tilde{\xi}, r_{0}\right)\right)$, thus, taking $C:=\sup _{\lambda \in \mathbb{R}} C(n, \lambda)$, we have that $B_{g}\left(\tilde{\xi}, r_{0}\right)$ is directionally $(\varepsilon, C)$ limited.

The notion of directionally limited sets, permits to generalize a classical covering Theorem, known as the Besicovitch coverig theorem, to more general settings, as a closed balls of a Riemannian manifold with regular enough metric (c.f. [Mag12] Theorem 5.1 for the Euclidean case).

Theorem 2.1.4 (Federer-Besicovitch covering theorem). Let $\mathcal{B}:=\left\{\bar{B}_{d}\left(x_{\alpha}, r_{\alpha}\right)\right\}_{\alpha \in \Lambda}$ a family of closed balls such that $\left\{x_{\alpha}\right\}_{\alpha \in \Lambda}=A$ is a directionally $(\delta, C)$ limited subset of $X$, and $0<r_{\alpha}<\delta$ for all $\alpha \in \Lambda$, then there are $2 C+1$ disjointed subfamilies $\mathcal{G}_{1}, \ldots, \mathcal{G}_{2 C+1}$ of $\mathcal{B}$ such that

$$
A \subset \bigcup_{i=1}^{2 C+1} \bigcup_{B \in \mathcal{G}_{i}} B .
$$

Corollary 2.1.4.1 (Vitali's symmetric property). Let $\mu$ be a Radon measure on $X$, and $\mathcal{B}:=$ $\left\{\bar{B}_{d}\left(x_{\alpha}, r_{\alpha}\right)\right\}_{\alpha \in \Lambda}$ a collection of closed non-degenerate balls such that $A=\left\{x_{\alpha}\right\}_{\alpha \in \Lambda}$ is $\mu$-measurable, and directionally $(\delta, C)$ limited, and, for every $x_{\alpha} \in A$,

$$
\inf _{\alpha \in \Lambda} \operatorname{diam}\left(\bar{B}_{d}\left(x_{\alpha}, r_{\alpha}\right)\right)=0,
$$

then there exists a countable disjoint subfamily $\mathcal{G}:=\left\{\bar{B}_{d}\left(x_{\beta}, r_{\beta}\right)\right\}_{\beta \in \Lambda^{\prime} \subset \Lambda}$ of $\mathcal{B}$ such that

$$
\mu\left(A \backslash \bigcup_{\beta \in \Lambda^{\prime}} \bar{B}_{d}\left(x_{\beta}, r_{\beta}\right)\right)=0 .
$$

Definition 2.1.6. Let $(X, d)$ be locally compact and separable metric space and a $\mu$ a Radon measure on $X$ satisfying the hypothesis of the Corollary 2.1.4.1, we say that $X$ satisfying has the Vitali's symmetric property with respect to $\mu$.

Remark 2.1.1. Since we have seen that given a Riemannian manifold $\left(M^{n}, g\right)$ with $C^{2}$ (or more regular) metric the geodesic balls $\bar{B}_{g}(\xi, r)$ with $r<i n j_{\left(M^{n}, g\right)}(\xi)$ are directionally $(\varepsilon, C)$ limited for suitable $\varepsilon$ and $C(n)$, for each $\xi \in \bar{B}_{g}\left(\tilde{\xi}, i n j_{\left(M^{n}, g\right)}(\tilde{\xi})\right)$, the Theorem 2.1.4 is valid, and then $\left(\bar{B}_{g}\left(\tilde{\xi}, \operatorname{inj}_{\left(M^{n}, g\right)}(\tilde{\xi})\right)\right)$ satisfy the Vitali's symmetric property for a regular enough Radon measure $\mu$ on $M$. In fact every compact subset of a Riemannian manifold with metric of class greater than or equal than 2, c.f. [Fed69] 2.9.

\subsubsection{Densities and Differentiation of measures}

Definition 2.1.7. Let $X$ be a metric space, for any outer measure $\mu$ on $X$, any subset $A \subset X$, and any point $x \in X$, we define the $n$-dimensional upper and lower densities $\Theta^{* n}(\mu, A, x), \Theta_{*}^{n}(\mu, A, x)$ 
by

$$
\begin{aligned}
\Theta^{* n}(\mu, A, x) & =\limsup _{\rho \downarrow 0} \frac{\mu(A \cap B(x, \rho))}{\omega_{n} \rho^{n}} \\
\Theta_{*}^{n}(\mu, A, x) & =\limsup _{\rho \downarrow 0} \frac{\mu(A \cap B(x, \rho))}{\omega_{n} \rho^{n}}
\end{aligned}
$$

If both limits exists and coincides, the common value is called the $n$-dimensional density of $\mu$ at $x \in A$, and is denoted by $\Theta^{n}(\mu, A, x)$ In case $A=X$ we simply write $\Theta^{* n}(\mu, x)$ and $\Theta_{*}^{n}(\mu, x)$ to denote these quantities, so that $\Theta^{* n}(\mu, A, x)=\Theta^{* n}\left(\mu\llcorner A, x), \Theta_{*}^{n}(\mu, A, x)=\Theta_{*}^{n}(\mu\llcorner A, x)\right.$.

Definition 2.1.8 (Upper and lower $\mu$-densities). Let $\mu$ and $\nu$ be Radon measures on $X$, metric space, the upper $\mu$ density and the lower $\mu$ density of $\nu$ are functions

$$
\begin{aligned}
& D_{\mu}^{+} \nu: \operatorname{spt} \mu \rightarrow[0, \infty], \\
& D_{\mu}^{-} \nu: \operatorname{spt} \mu \rightarrow[0, \infty],
\end{aligned}
$$

defined respectively as

$$
\begin{aligned}
& D_{\mu}^{+} \nu(x):=\limsup _{r \downarrow 0} \frac{\nu(\bar{B}(x, r))}{\mu(\bar{B}(x, r))}, \quad x \in \operatorname{spt} \mu, \\
& D_{\mu}^{-} \nu(x):=\liminf _{r \downarrow 0} \frac{\nu(\bar{B}(x, r))}{\mu(\bar{B}(x, r))}, \quad x \in \operatorname{spt} \mu .
\end{aligned}
$$

If the two limits exists and are finite, then we denote by $D_{\mu} \nu(x)$ their common value and call it the $\mu$-density on $\nu$ at $x$. Thus we have defined a function

$$
D_{\mu} \nu:\left\{x \in \operatorname{spt} \mu: D_{\mu}^{-} \nu=D_{\mu}^{+} \nu\right\} \rightarrow[0, \infty] .
$$

The following result is a classic result concerning with differentiation of Radon measures, and for the proof can be found in [Fed69] in full generality, or in [Sim83]. Once we have discuss how geodesic balls in a Riemannian manifold of class greater than or equal than 2 satisfies the Vitali's symmetric property in the full generality, the Simons approach is what we follow.

Theorem 2.1.5 (Lebesgue-Besicovitch-Federer differentiation Theorem). Let $\mu$ and $\nu$ are Radon measures on $X$, a metric space satisfying the Vitali's symetric property for $\nu$. Then $D_{\mu} \nu$ is defined $\mu$-a.e. on $X, D_{\mu} \nu \in L_{l o c}^{1}(X, \mu)$, and, in fact $D_{\mu} \nu$ is a Borel measure on $X$. Furthermore

$$
\nu=\left(D_{\mu} \nu\right) \mu+\nu_{\mu}^{s}, \quad \text { on } \mathcal{M}(\mu),
$$

where the Radon measure $\nu_{\mu}^{s}$ is concentrated on the Borel set

$$
\begin{aligned}
Y & =\mathbb{R}^{n} \backslash\left\{x \in \operatorname{spt} \mu: D_{\mu}^{+} \nu(x)<\infty\right\} \\
& =\left(\mathbb{R}^{n} \backslash \operatorname{spt} \mu\right) \cup\left\{x \in \operatorname{spt} \mu: D_{\mu}^{+} \nu(x)=\infty\right\},
\end{aligned}
$$

in particular; $\nu_{\mu}^{s} \perp \mu$.

\subsubsection{Grassmanian manifold}

To end this "beginners guide" in geometric measure theory, we look up to the Grassmanian manifold (the fact that this is in fact a differentiable manifold, is a well know exercise on topology of manifolds, and can be founded in [War83]) and study their metric properties in the particular case in which we have $k$ dimensional subspaces of a tangent space $T_{x} M^{n}$ of an $n$ dimensional manifold $M$. 
Definition 2.1.9. Let $\left(M^{n}, g\right)$ a n-dimensional Riemmanian manifold, the space of all $k$-dimensional $(k<n)$ subspaces of $T_{x} M$, for $x \in M$ fixed will be denoted as $\operatorname{Gr}\left(k, T_{x} M\right)$ endowed with the usual topology.

Since for $x$ fixed $T_{x} M \simeq \mathbb{R}^{n}$ the topology is the same. Suppose $S \in \operatorname{Gr}\left(k, T_{x} M\right)$ We will use $p_{S}$ to denote orthogonal projection of $T_{x} M$ onto $S$. That is, $p_{S} \in \operatorname{Hom}\left(T_{x} M, T_{x} M\right)$ is defined as

$$
p_{S}(u)=\sum_{i=1}^{k}\left\langle u, \tau_{i}\right\rangle_{g} \tau_{i},
$$

where $u \in T_{x} M$ for $x$ fixed, and $\left\{\tau_{1}, \ldots, \tau_{k}\right\}$ is an orthonormal basis for $S \in \operatorname{Gr}\left(k, T_{\xi} M\right)$.

Then, the associated matrix $\left(e^{i j}\right)_{i, j=1}^{n}$ to $p_{S}$ is defined as

$$
e^{i j}=\left\langle e_{i}, \tau_{j}\right\rangle
$$

where, as before $\left\{\tau_{1}, \ldots, \tau_{k}\right\}$ is an onrthonormal basis for $S$, and $\left\{e_{1}, \ldots, e_{n}\right\}$ is an orthonormal basis for $T_{x} M$. Notice that $p_{S}$ satisfies

$$
p_{S} \circ p_{S}=p_{S}, \quad\left(p_{S}\right)^{*}=p_{S} \text { and } \operatorname{Im}\left(p_{S}\right)=p_{S} .
$$

Therefore $\left(e^{i j}\right)_{i, j=1}^{n}$ satisfies

$$
\left(e^{i j}\right)^{2}=e^{i j} \text { and trace }\left(e^{i j}\right)_{i, j=1}^{n}=n .
$$

On the other hand, we can induce an inner product on $\operatorname{Hom}\left(T_{x} M, T_{x} M\right)$ as

$$
L_{1} \bullet L_{2}=\operatorname{trace}\left(L_{1}^{*} \circ L_{2}\right),
$$

and this inner product naturally induces a norm, denoted by $|\cdot|$. Notice that, if we consider the operator norm on $\operatorname{Hom}\left(T_{x} M, T_{x} M\right)$ defined as

$$
\|L\|=\sup \left\{|L(v)|_{g}: v \in T_{x} M \text { and }|v| \leq 1\right\},
$$

we have

$$
\|L\| \leq|L| \leq n^{1 / 2}\|L\|
$$

\subsection{Riemannian Geometry}

In this section the basics on Riemannian geometry to prove the Lemma 3.6 of [HS74] are covered. Such result is a comparison theorem for the Hessian of the distance function (naturally defined in an $n$-dimensional Riemannian manifold). Because this result is a comparison of the partial divergence of a radial vector field, we define,

Definition 2.2.1. Let $\left(M^{n}, g\right)$ be an $n$-dimensional Riemannian manifold, with Levi-Civita connection $\nabla$, let $T \in \operatorname{Gr}\left(k, T_{\xi} M\right)$ and $\left\{e_{1}, \ldots, e_{k}\right\}$ an orthonormal basis for $T$. The partial divergence of a vector field $X$ over $T$ is defined as

$$
\operatorname{div}_{T} X(x)=\sum_{i=1}^{n}\left\langle\nabla_{e_{i}} X(x), e_{i}\right\rangle_{g} .
$$

Readily follows that, in the case in which $T=T_{\xi} M$ for some $\xi \in M$, this partial divergence coincide with the classical definition of the divergence of a vector field.

Lemma 2.2.1. Let $\left(M^{n}, g\right)$ be a complete $n$-dimensional Riemannian manifold, with Levi-Civita connection $\nabla$, let $b \in \mathbb{R}$ such that $S e c_{g} \leq b$, let $0<r_{0}<i n j(M, g)$ such that br $r_{0}<\pi$ and let $\xi \in M$. 
Then, setting $u(x):=r_{\xi}(x):=\operatorname{dist}_{(M, g)}(x, \xi)$,

$$
\operatorname{div}_{T}(u \nabla u)(x) \geq k u(x) \cot _{b}(u(x)),
$$

for all $x \in B_{g}\left(\xi, r_{0}\right):=\left\{x \in M: \operatorname{dist}_{(M, g)}(x, \xi) \leq r_{0}\right\}$, and for all $T \in \operatorname{Gr}\left(k, T_{\xi} M\right)$.

Remark 2.2.1. First to all, recall that, given a Riemannian manifold $(M, g)$, it is possible to this define a distance function induced by the metric $g$ as follows:

$$
\operatorname{dist}_{(M, g)}(p, q)=\inf \left\{\ell_{g}(\gamma): \gamma:[a, b] \rightarrow M \text { smooth curve with } \gamma(a)=p \text { and } \gamma(b)=q\right\},
$$

where $\ell_{g}(\gamma)$ denotes the length of the curve $\gamma$, which in a coordinate patch $U \subset M$ is defined as

$$
\ell_{g}(\gamma)=\int_{a}^{b}|\dot{\gamma}(t)|_{g} d t=\int_{a}^{b} \sqrt{\langle\dot{\gamma}(t), \dot{\gamma}(t)\rangle_{g}} d t=\int_{a}^{b} \sqrt{g_{i j}(\gamma(t)) \dot{\gamma}_{i}(t) \dot{\gamma}_{j}(t)} d t
$$

In general such distance is not well defined, but, if we consider such distance function on $B_{g}\left(\xi, r_{0}\right)$ is well defined since the exponential map $\exp _{\xi}:\left\{v \in T_{\xi} M:|v|_{g}<r_{0}\right\} \rightarrow M$ is a difeomorphism.

On the other hand, the result involves the quantity $\cot _{b}(s)$ which is defined as:

$$
\cot _{b}(s)=\frac{c s_{b}(s)}{s n_{b}(s)}
$$

where $\operatorname{sn}_{b}(s)$ is the unique solution to

$$
\begin{cases}x^{\prime \prime}(s)+b x(s) & =0 \\ x(0) & =0 \\ x^{\prime}(0) & =1,\end{cases}
$$

and $c s_{b}(s)=s n_{b}^{\prime}(s)$.

By the classical theory of second order ODE, to obtain explicit solutions to (2.2), by analyze the characteristic polynomial associated to it, namely

$$
\lambda^{2}+b=0 .
$$

Then, we have three cases

\section{Case 1:}

Assume $b=0$, then

$$
x^{\prime \prime}(s)=0,
$$

then, the solution is

$$
x(s)=s,
$$

and

$$
s \cot _{b}(s)=1 .
$$

\section{Case 2:}

Assume $b>0$, then

$$
\lambda= \pm \sqrt{b} i,
$$


then the solution to $(2.2)$ is

$$
x(s)=\frac{\sin (\sqrt{b} t)}{\sqrt{b}},
$$

so,

$$
s \cot _{b}(s)=s \sqrt{b} \cdot \frac{\cos (\sqrt{b} s)}{\sin (\sqrt{b} s)} .
$$

And an asymptotic analysis when $s \rightarrow 0$ gives

$$
\begin{aligned}
s \cot _{b}(s) & \sim \sqrt{b} s\left(\frac{1-\frac{b s^{2}}{2 !}+O\left(s^{4}\right)}{\sqrt{b} s-\frac{b \sqrt{b} s^{3}}{3 !}+O\left(s^{5}\right)}\right) \\
& \sim \frac{1-\frac{b s^{2}}{2 !}+O\left(s^{4}\right)}{1-\frac{b s^{2}}{3 !}+O\left(s^{4}\right)} \\
& \sim\left(1-\frac{b s^{2}}{2 !}+O\left(s^{4}\right)\right)\left(1-\frac{b s^{2}}{3 !}+O\left(s^{4}\right)\right) \\
& \sim 1+\frac{b s^{2}}{3 !}-\frac{b s^{2}}{2 !}+O\left(s^{4}\right) \\
& \sim 1+\left(\frac{b}{6}-\frac{b}{2}\right) s^{2}+O\left(S^{4}\right) \\
& \sim 1-\frac{s^{2}}{3}+O\left(s^{4}\right) \\
& \sim 1-O\left(s^{2}\right)
\end{aligned}
$$

Case 3:

Assume $b<0$, then

$$
\lambda= \pm \sqrt{|b|},
$$

then the solution to (2.2) is

$$
x(s)=\frac{e^{\sqrt{|b|} s}-e^{-\sqrt{|b|} s}}{2 \sqrt{|b|}}=\frac{\sinh (\sqrt{|b|} s)}{\sqrt{|b|}},
$$

so,

$$
s \cot _{b}(s)=\sqrt{|b|} s \cdot \frac{\cosh (\sqrt{|b|} s)}{\sinh (\sqrt{|b|} s)} .
$$


As in the previous case, an asymptotic analysis when $s \rightarrow 0$ gives

$$
\begin{aligned}
s \cot _{b}(s) & \sim \sqrt{b} s\left(\frac{1+\frac{b s^{2}}{2 !}+O\left(s^{4}\right)}{\sqrt{b} s+\frac{b \sqrt{b} s^{3}}{3 !}+O\left(s^{5}\right)}\right) \\
& \sim \frac{1+\frac{b s^{2}}{2 !}+O\left(s^{4}\right)}{1+\frac{b s^{2}}{3 !}+O\left(s^{4}\right)} \\
& \sim\left(1+\frac{b s^{2}}{2 !}+O\left(s^{4}\right)\right)\left(1-\frac{b s^{2}}{3 !}+O\left(s^{4}\right)\right) \\
& \sim 1-\frac{b s^{2}}{3 !}+\frac{b s^{2}}{2 !}+O\left(s^{4}\right) \\
& \sim 1+\left(\frac{b}{2}-\frac{b}{6}\right) s^{2}+O\left(S^{4}\right) \\
& \sim 1+\frac{s^{2}}{3}+O\left(s^{4}\right) \\
& \sim 1+O\left(s^{2}\right) .
\end{aligned}
$$

The asymptotic analysis above will play a key role later in the Section 4.

In order to prove the Lemma 2.2.1, we will use the well known Rauch Theorem and a Hessian comparison theorem derived from it.

\subsubsection{Lie Derivatives and the Metric}

This section is inspired in the Chapter 2 of [Pet06], so the reader who is not familiarized with this, is encouraged to seek for the proofs there.

Let $X$ be a vector field and $F^{t}$ the flow associated to $X$ on a smoothmanifold $M$ (which locally always exists). Hence, $F^{t}(p)$ is well defined for $t$ small and so, the curve $t \mapsto F^{t}(p)$ is the integral curve for $X$ that passes through $p$ at $t=0$. The Lie derivative of a tensor $T$ in the direction of $X$ is defined as the first-order term in a suitable expansion of $T$ when moved by the flow of $X$. However, the precise formula depends on what kind of tensor is $T$.

Definition 2.2.2. Let $\left(M^{n}, g\right)$ an n-dimensional Riemannian manifold with Levi-Civita connection $\nabla$, and let $T$ a $(0, k)$-tensor. Define the Lie derivative of $T$ along the vector field $X$ as:

$$
\left(\nabla_{X} T\right)\left(Y_{1}, \ldots, Y_{k}\right)=\lim _{t \rightarrow 0} \frac{\left(F^{t}\right)^{*} T-T}{t},
$$

where $\left(F^{t}\right)^{*} T\left(Y_{1}, \ldots, Y_{k}\right)=T\left(D F^{t}\left(Y_{1}\right), \ldots, D F^{t}\left(Y_{k}\right)\right)$ is the pull-back of $T$ by $F^{t}$.

The following proposition shows that the Lie derivative is in fact a derivation

Proposition 2.2.1. Let $\left(M^{n}, g\right)$ an n-dimensional Riemannian manifold with Levi-Civita connection $\nabla$.

(i) If $X$ is a vector field and $T$ a $(0, k)$-tensor on $M$, then

$$
\left(\nabla_{X} T\right)\left(Y_{1}, \ldots, Y_{k}\right)=\nabla_{X}\left(T\left(Y_{1}, \ldots, Y_{k}\right)\right)-\sum_{i=1}^{k} T\left(Y_{1}, \ldots, \nabla_{X} Y_{i}, \ldots, Y_{k}\right)
$$

(ii) If $T_{1}$ and $T_{2}$ be $\left(0, k_{i}\right)$-tensors, then

$$
\nabla_{X}\left(T_{1} \cdot T_{2}\right)=\left(\nabla_{X} T_{1}\right) \cdot T_{2}+T_{1} \cdot\left(\nabla_{X} T_{2}\right)
$$


(iii) If $T$ is a $(0, k)$-tensor and $f: M \rightarrow \mathbb{R}$ a function, then

$$
\nabla_{f X} T\left(Y_{1}, \ldots, Y_{k}\right)=f \nabla_{X} T\left(Y_{1}, \ldots, Y_{k}\right)+\sum_{i=1}^{k}\left(\nabla_{Y_{i}} f\right) T\left(Y_{1}, \ldots, X, \ldots, Y_{k}\right) .
$$

The Lie derivative of a tensor gives us the notion of the Hessian of a function $f: M \rightarrow \mathbb{R}$ on a Riemannian manifold as a $(0,2)$-tensor:

Definition 2.2.3. Let $\left(M^{n}, g\right)$ an n-dimensional Riemannian manifold, with Levi-Civita connection $\nabla$. The Hessian of a function $f: M \rightarrow \mathbb{R}$ is defined as the $(0,2)$-tensor Hess $f$ such that:

$$
\text { Hess } f(X, Y)=\frac{1}{2}\left(\nabla_{\nabla f} g\right)(X, Y)
$$

where $\nabla f:=\operatorname{grad}(f)$ is the vector field satisfying $\langle v, \nabla f\rangle_{g}=d f(v)$ for all $v \in T M$.

The special case in which $f \equiv r_{\xi}$ will be studied in the next section trough the machinery of the Jacobi fields. On the other hand we can analyse right now the Hessian of the square of the distance function in terms of the Hessian of the distance, this calculations will enter at the scene in the proof of the Lemma 2.2.1. Since the Lie derivative of a tensor is in fact a derivation, we have, in view of the Proposition 2.2.1, that, given $X$ and $Y$ vector fields on $M$

$$
\text { Hess } \begin{aligned}
\frac{1}{2} r_{\xi}^{2}(X, Y) & =\frac{1}{2}\left(\nabla_{\nabla \frac{1}{2} r_{\xi}^{2}} g\right)(X, Y) \\
& =\frac{1}{2}\left(\nabla_{r_{\xi} \nabla r_{\xi}} g\right)(X, Y) \\
& =\frac{1}{2}\left(r_{\xi} \nabla \nabla r_{\xi} g(X, Y)+\nabla_{X} r_{\xi} g\left(\nabla r_{\xi}, Y\right)+\nabla_{Y} r_{\xi} g\left(X, \nabla r_{\xi}\right)\right) \\
& =r_{\xi} \operatorname{Hess} r_{\xi}(X, Y)+\frac{1}{2}\left(\nabla_{X} r_{\xi} g\left(\nabla r_{\xi}, Y\right)+\nabla_{Y} r_{\xi} g\left(X, \nabla r_{\xi}\right)\right) .
\end{aligned}
$$

Therefore, if $\left\{E_{1}, \ldots, E_{k}\right\} \subset T M$ is an orthonormal frame, letting $E_{i}(\xi):=e_{i} \in T_{\xi} M$ for all $i=1, \ldots n$, then $\left\{e_{1}, \ldots, e_{k}\right\}$ is an orthonormal basis of some $T \in \operatorname{Gr}\left(k, T_{\xi} M\right)$ and

$$
\operatorname{Hess} \frac{1}{2} r_{\xi}^{2}\left(e_{i}, e_{i}\right)=r_{\xi} \operatorname{Hess} r_{\xi}\left(e_{i}, e_{i}\right)+\nabla_{e_{i}} r_{\xi}\left\langle\nabla r_{\xi}, e_{i}\right\rangle_{g}=r_{\xi} \operatorname{Hess} r_{\xi}\left(e_{i}, e_{i}\right)+\left\langle\nabla r_{\xi}, e_{i}\right\rangle_{g}^{2} \text {. }
$$

\subsubsection{Jacobi Fields and Rauch Comparison Theorem}

Definition 2.2.4. Let $\gamma: I \rightarrow M$ be geodesic. A vector field $X$ along $\gamma$ is called a Jacobi field if

$$
\nabla_{\frac{d \gamma}{d t}} \nabla_{\frac{d \gamma}{d t}} X+R(X, \dot{\gamma}) \dot{\gamma}=0
$$

As an abbreviation, we shall sometimes write

$$
\dot{X}=\nabla_{\frac{d \gamma}{d t}} X, \quad \ddot{X}=\nabla_{\frac{d \gamma}{d t}} \nabla_{\frac{d \gamma}{d t}} X
$$

then the above expression becomes

$$
\ddot{X}+R(X, \dot{\gamma}) \dot{\gamma}=0,
$$

where $R$ stands for the curvature tensor.

The following lemma shows the existence and uniqueness of Jacobi fields with given initial data. For this, as in the case of geodesics, the Jacobi equation will be interpreted as a system of $(n=\operatorname{dim} M)$ linear second order ODEs.

Lemma 2.2.2. Let $\gamma:[a, b] \rightarrow M$ be a geodesic. For any $v, w \in T_{\gamma(a)} M$, there exists a unique 
Jacobi field $X$ along $\gamma$ with

$$
X(p)=v, \dot{X}(p)=w .
$$

Since the existence and uniques of Jacobi fields are ensure for given initial values of it along a geodesic, a natural question is how to construct a Jacobi field along a given geodesic. the next theorem shows how to construct Jacobi fields.

Theorem 2.2.3. Let $\gamma:[0, T] \rightarrow M$ be geodesic, defined up to a time $T>0$. Let $c(t, s)$ be a variation of $\gamma(t)$, i.e.

$$
\gamma(\cdot, \cdot):[0, T] \times]-\varepsilon, \varepsilon[\rightarrow M,
$$

for which all curves $\gamma(\cdot, s)=: \gamma_{s}(\cdot)$ are geodesics, too. Then,

$$
X(t):=\left.\frac{\partial}{\partial s} c(t, s)\right|_{s=0}
$$

is a Jacobi field along $\gamma(t)=\gamma_{0}(t)$.

Conversely, every Jacobi field along $\gamma(t)$ may be obtained in this way, i.e. by a variation of $\gamma(t)$ through geodesics.

Let $\gamma(t, s)=\gamma_{s}(t)$ be a family of geodesics parametrized by $s$, i.e.

$$
\nabla_{\frac{\partial \gamma}{\partial t}} \frac{\partial \gamma}{\partial t}(t, s)=0 \quad \text { for all } s
$$

Then also

$$
\nabla_{\frac{\partial \partial}{\partial s}} \nabla_{\frac{\partial}{\partial t}} \frac{\partial \gamma}{\partial t}(t, s)=0
$$

and this implies that $X(t)=\left.\frac{\partial c}{\partial s}(t, s)\right|_{s=0}$ satisfies the Jacobi equation. Consequently, the Jacobi equation is the linearization of the equation for geodesic curves. Thus, If a particular proper variation of a geodesic through geodesics is given, then also the 2nd derivative of the length and energy functionals with respect to the family parameter vanish.

Corollary 2.2.3.1. Let $\gamma:[0, T] \rightarrow M$ be a geodesic, defined up a given time $T>0$ and $p=\gamma(0)$, i.e.

$$
\gamma(t)=\exp _{p} t \dot{\gamma}(0)
$$

For $w \in T_{p} M$, the Jacobi field $X$ along $\gamma$ with $X(0)=0, \dot{X}(0)=w$ then is given by:

$$
X(t)=\left(D \exp _{p}\right)_{t \dot{\gamma}(0)}[t w]
$$

where

$$
\begin{aligned}
\left(D \exp _{p}\right)_{t \dot{\gamma}(0)}: T_{t \dot{\gamma}(0)}\left(T_{p} M\right) & \rightarrow T_{\exp _{p}(t \dot{\gamma}(0))} M \\
t v & \mapsto\left(D \exp _{p}\right)_{t \dot{\gamma}(0)}[t v] .
\end{aligned}
$$

Now, let $\left(M^{n}, g\right)$ be an $n$-dimensional Riemannian manifold with curvature $K$ satisfying, for $b^{ \pm} \in \mathbb{R}$

$$
b^{-} \leq K \leq b^{+}
$$

and assume $b^{-} \leq 0, b^{+} \geq 0$. We must estimate a Jacobi field in $M$ by the Jacobi field in $H^{n}\left(-b^{-}\right)$ (The space form with negative curvature) from above, with initial values of same lengths, and from below by the corresponding one in $S^{n}\left(b^{+}\right)$. Thus the distance between geodesics and also the derivative of the exponential map of $M$ can be controlled by the geometry of the model spaces $H^{n}\left(-b^{-}\right)$and $S^{n}\left(b^{+}\right)$. Instead of consider Jacobi fields with vanishing tangential component we shall assume $b^{-} \leq 0$ and $b^{+} \geq 0$. 
As in Remark 2.2.1, we put for $b \in \mathbb{R}$

$$
c n_{b}(t):= \begin{cases}\cos (\sqrt{b} t) & \text { if } b>0 \\ 1 & \text { if } b=0 \\ \cosh (\sqrt{-b} t) & \text { if } b<0\end{cases}
$$

and

$$
s n_{b}(t):= \begin{cases}\frac{1}{\sqrt{b}} \sin (\sqrt{b} t) & \text { if } b>0 \\ t & \text { if } b=0 \\ \frac{1}{\sqrt{-b}} \sinh (\sqrt{-b} t) & \text { if } b<0\end{cases}
$$

As seen in the remark 2.2.1, these functions are solutions of a second order ODE, which coincides with the Jacobi equation for constant sectional curvature $b$, namely

$$
\ddot{f}(t)+b f(t)=0
$$

with initial values $f(0)=1, \dot{f}(0)=0$,respectively $f(0)=0, \dot{f}(0)=1$. Henceforth $\gamma(t)$ will always be a geodesic on $M$ parametrized by arc length, i.e. satisfying

$$
|\dot{\gamma}|_{g} \equiv 1
$$

Let $J(t)$ be a Jacobi field along $\gamma(t)$.

Theorem 2.2.4 (Rauch Comparison Theorem I ). Suppose $K \leq b$, and $\gamma$ be parametrized by arc length. Assume either $b \geq 0$ or that the tangential component of $J$ is identically zero along $\gamma$.

Let $f_{\mu}:=|J(0)|_{g} c_{\mu}+|\dot{J}|_{g}(0) s_{\mu}$ solve

$$
\ddot{f}+\mu f=0
$$

with $f(0)=|J(0)|_{g}$, and $\dot{f}(0)=|J|_{g}(0)$,

If

$$
f_{\mu}(t)>0 \quad \text { for } 0<t<\tau
$$

then

$$
\begin{aligned}
\langle J, \dot{J}\rangle_{g} f_{\mu} & \geq\langle J, J\rangle_{g} \dot{f}_{\mu} \text { on }[0, \tau], \\
1 \leq \frac{\left|J\left(t_{1}\right)\right|_{g}}{f_{\mu}\left(t_{1}\right)} & \leq \frac{\left|J\left(t_{2}\right)\right|_{g}}{f_{\mu}\left(t_{2}\right)}, \text { if } 0<t_{1} \leq t_{2}<\tau, \\
|J(0)|_{g} c n_{b}(t)+|J|_{g}(0) s n_{b}(t) & \leq|J(t)|_{g} \text { for } 0 \leq t \leq \tau .
\end{aligned}
$$

The proof of this result can be found in [Jos17] Theorem 4.5.1, from where this version was taken, as well as the next result, which is the case in which the sectional curvature is bounded from bellow (c.f. [Jos17] Theorem 4.5.2)

Theorem 2.2.5 (Rauch Comparison Theorem II). Assume $b^{-} \leq K \leq b^{+}$and either $b^{-} \leq 0$ or that the tangential component of $J$ is identically zero along $\gamma$; also, as always, $\gamma$ is paramatrized by arc length. Moreover, let $J(0)$ and $\dot{J}(0)$ be linearly dependent.

Assume

$$
\left.s n_{\frac{1}{2}\left(b^{-}+b^{+}\right)}>0 \text { on }\right] 0, \tau[.
$$

Then, for $0 \leq t \leq \tau$

$$
|J(t)|_{g} \leq|J(0)|_{g} c n_{b^{-}}(t)+|J|_{g}(0) s n_{b^{+}}(t) .
$$

\subsubsection{Geometric Applications of Jacobi Fields Estimates}

Finally, it is time to put all together, in order to prove the Lemma 2.2.1, before that, we start by proving an estimate on the Hessian of the distance function $r_{\xi}$ as a consequence of the Rauch 
comparison theorems, and for clarifying purposes we will prove it, following the proof of the Theorem 4.6.1 of [Jos17], where the reader will find this comming theorem.

Theorem 2.2.6. Let the exponential map $\exp _{p}: T_{p} M \rightarrow M$ be a diffeomorphism on $\left\{v \in T_{p} M:|v|_{g} \leq \rho\right\}$.

Let the curvature of $M$ in the ball

$$
B_{g}(\xi, \rho):=\left\{q \in M: d_{g}(\xi, q) \leq \rho\right\}
$$

satisfying

$$
b^{-} \leq K \leq b^{+},
$$

with $b^{-} \leq 0, b^{+} \geq 0$, and suppose

$$
\rho<\frac{\pi}{2 \sqrt{b^{+}}} \text {in case } b^{+}>0
$$

Let $r_{\xi}(x):=d_{g}(x, \xi)$. Then $\frac{1}{2} r_{\xi}^{2}$ is smooth on $B_{g}(\xi, \rho)$ and satisfies

$$
\nabla\left(\frac{1}{2} r_{\xi}^{2}\right)(x)=-\exp _{x}^{-1}(\xi)
$$

and therefore

$$
\begin{gathered}
\left|\nabla\left(\frac{1}{2} r_{\xi}^{2}\right)(x)\right|_{g}=r_{\xi}(x) . \\
r_{\xi}(x) \cot _{b^{+}}\left(r_{\xi}(x)\right)|v|_{g}^{2} \leq \operatorname{Hess}\left(\frac{1}{2} r_{\xi}\right)(v, v) \leq r(x) \cot _{\left(-b^{-}\right)}(r(x))|v|_{g}^{2},
\end{gathered}
$$

for all $x \in B_{g}(\xi, \rho), v \in T_{x} M$ and $\cot _{b^{ \pm}}$be defined as in the remark 2.2.1

Proof. Since $\nabla\left(\frac{1}{2} r_{\xi}^{2}\right)(x)$ is, by definition, orthogonal to the level surfaces of $\frac{1}{2} r_{\xi}^{2}$, and those are the spheres

$$
S_{g}(\xi, R):=\left\{q \in M: d_{g}(\xi, q)=R\right\}=\exp _{\xi}\left\{v \in T_{\xi} M:\|v\|=R\right\},
$$

where $R<\rho$, we have

$$
\nabla\left(\frac{1}{2} r_{\xi}^{2}\right)(x)=-\exp _{x}^{-1} \xi
$$

and, in particular, $\nabla\left(\frac{1}{2} r_{\xi}^{2}\right)$ has length $d_{g}(x, \xi)$, proving the first assertion (2.6).

On the other hand, since Hess $\left(\frac{1}{2} r_{\xi}^{2}\right)$ is symmetric, it can hence be diagonalized, without loss of generality, it thus suffices to prove (2.7) for each eigen direction $v$ of Hess $\left(\frac{1}{2} r_{\xi}^{2}\right)$. Let $\eta(s)$ be the curve in $M$ with $\eta(0)=x, \eta^{\prime}(0)=v$

$$
\gamma(t, s):=\exp _{\eta(s)}\left(t \exp _{\eta(s)}^{-1} \xi\right)
$$

in particular $\gamma(0, s)=\eta(s), \eta(1, s) \equiv \xi$.

Then,

$$
\nabla\left(\frac{1}{2} r_{\xi}^{2}\right)(\gamma(s))=-\left.\frac{\partial}{\partial t} \gamma(t, s)\right|_{l=0},
$$


hence

$$
\begin{aligned}
\nabla_{v}\left(\nabla\left(\frac{1}{2} r_{\xi}^{2}\right)(x)\right) & =-\left.\nabla \frac{\partial}{\partial s} \frac{\partial}{\partial t} \gamma(t, s)\right|_{t=0, s=0} \\
& =-\left.\nabla \frac{\partial}{\partial t} \frac{\partial}{\partial s} \gamma(t, s)\right|_{t=0, s=0},
\end{aligned}
$$

therefore,

$$
J(t):=\frac{\partial}{\partial s} \gamma(t, s)_{\mid s=0},
$$

is a Jacobi field along the geodesic, parametrized by arc length, from $x$ to $\xi$ with $J(0)=\dot{\gamma}(0)=$ $v, J(1)=0 \in T_{p} M$, thus

$$
\nabla_{v}\left(\nabla\left(\frac{1}{2} r_{\xi}^{2}\right)(x)\right)=-\dot{J}(0)
$$

i.e.

$$
\operatorname{Hess}\left(\frac{1}{2} r_{\xi}^{2}\right)(v, v)=\left\langle\nabla_{v}\left(\nabla\left(\frac{1}{2} r_{\xi}^{2}\right)\right), v\right\rangle_{g}=-\langle\dot{J}(0), J(0)\rangle_{g}
$$

since $v$ is an eigen direction of Hess $\left(\frac{1}{2} r_{\xi}^{2}\right), \nabla_{v}\left(\frac{1}{2} r_{\xi}^{2}\right)$ and $v$, i.e. $\dot{J}(0)$ and $J(0)$ are linearly dependent. Finally (2.4) and (2.5) imply for $t=1(J(1)=0)$ (notice that we are assuming that the geodesic which $J$ is been defied along to, is parametrized by arc length, this is not always true, see the remark below)

$$
|v|_{g} c n_{b^{+}}(r(x))+|J|_{g}(0) s n_{b^{+}}(r(x)) \leq 0 \leq|v|_{g} c n_{b^{-}}(r(x))+|J|_{g}(0) s n_{\left(-b^{-}\right)}(r(x)),
$$

thus,

$$
\begin{aligned}
|v|_{g} \cot _{b^{+}}(r(x))+|J|_{g}(0) & \leq 0, \quad \text { and } \\
|v|_{g} \cot _{\left(-b^{-}\right)}(r(x))+|J|_{g}(0) & \geq 0
\end{aligned}
$$

since,

$$
|J|_{g}^{\cdot}(0)=\left.\frac{d}{d t} \sqrt{\langle J(t), J(t)\rangle_{g}}\right|_{t=0}=\frac{\langle\dot{J}(0), J(0)\rangle_{g}}{|J(0)|_{g}}=\frac{\langle\dot{J}(0), J(0)\rangle_{g}}{r_{\xi}(x)|v|_{g}},
$$

then, from (2.8) and (2.9),

$$
r_{\xi}(x) \cot _{b^{+}}\left(r_{\xi}(x)\right)|v|_{g}^{2} \leq \operatorname{Hess}\left(\frac{1}{2} r_{\xi}\right)(v, v) \leq r(x) \cot _{\left(-b^{-}\right)}(r(x))|v|_{g}^{2},
$$

which completes the proof.

Remark 2.2.2. If we do not assume $|\dot{\eta}|_{g} \equiv 1$, (where $\eta$ is the geodesic from $x$ to $\xi$ ) in all the preceding estimates, $t$ has to be replaced by $t|\dot{\eta}|_{g}$ as argument of $s n_{b^{ \pm}}, c n_{b^{ \pm}}, f_{b^{ \pm}}$etc. Namely, let

$$
\tilde{\eta}(t)=\eta\left(\frac{t}{|\dot{\eta}|_{g}}\right)
$$

be the reparametrization of $\eta$ by arc length. Then $\tilde{J}(t)=J\left(\frac{t}{|\dot{\eta}|}\right)$ is the Jacobi field along $\tilde{\eta}$ with

$$
\tilde{J}(0)=J(0), \tilde{J}(0)=\frac{\dot{J}(0)}{|\dot{\eta}|_{g}}
$$


since $J$ satisfies the Jacobi equation, $\tilde{J}$ satisfies

$$
\ddot{\tilde{J}}+R(\tilde{J}, \dot{\tilde{\eta}}) \dot{\tilde{\eta}}=0
$$

Thus, estimates for $\tilde{J}$ yield corresponding estimates for $J$.

Finally, as promised, we will prove the Lemma 2.2.1, as a corollary of the theorem above, and the calculation in (2.3).

Proof of Lemma 2.2.1: First to all, notice that, since $r_{0}<i n j_{(M, g)}(\xi)$, we know that the exponential map:

$$
\exp _{\xi}:\left\{v:|v| \leq r_{0}\right\} \subset T_{\xi} M \rightarrow B_{g}\left(\xi, r_{0}\right) \subset M,
$$

is a diffeomorphism, and since by hypothesis $K:=S e c_{g} \leq b$ for some $b \in \mathbb{R}$ on $B_{g}\left(\xi, r_{0}\right)$ we are in the hypothesis of the Theorem 2.2.6.

Now, by (2.3) we know that, given $T \in \operatorname{Gr}\left(k, T_{\xi} M\right)$ and $\left\{e_{1}, \ldots, e_{k}\right\}$ an orthonormal basis for it,

$$
\begin{aligned}
\operatorname{div}_{T}(u \nabla u) & =\sum_{i=1}^{k}\left\langle\nabla_{e_{i}}(u \nabla u), e_{i}\right\rangle_{g} \\
& =\sum_{i=1}^{k}\left\langle\nabla_{e_{i}} u \cdot \nabla u, e_{i}\right\rangle_{g}+\left\langle u \nabla_{e_{i}}(\nabla u), e_{i}\right\rangle_{g} \\
& =u \sum_{k=1}^{n}\left\langle\nabla_{e_{i}}(\nabla u), e_{i}\right\rangle_{g}+\sum_{i=1}^{k}\left\langle\nabla u, e_{i}\right\rangle_{g}^{2} \\
& =u \operatorname{div}_{T} \nabla u+\sum_{i=1}^{k}\left\langle\nabla u, e_{i}\right\rangle_{g}^{2} \\
& =u \sum_{i=1}^{k} \frac{1}{2} \nabla_{\nabla u} g\left(e_{i}, e_{i}\right)+\sum_{i=1}^{k}\left\langle\nabla u, e_{i}\right\rangle_{g}^{2} \\
& =\sum_{i=1}^{k} u \operatorname{Hess} u\left(e_{i}, e_{i}\right)+\left\langle\nabla u, e_{i}\right\rangle_{g}^{2} \\
& =\sum_{i=1}^{k} \operatorname{Hess} \frac{1}{2} r_{\xi}^{2}\left(e_{i}, e_{i}\right),
\end{aligned}
$$

and, by the Theorem 2.2.6 (using the only left side of (2.7), and setting $b^{+}=b$ ), we have that

$$
\operatorname{Hess}\left(\frac{1}{2} r_{\xi}^{2}\left(e_{i}, e_{i}\right)\right)(x) \geq r_{\xi}(x) \cot _{b}\left(r_{\xi}(x)\right)
$$

Therefore

$$
\operatorname{div}_{T}(u \nabla u) \geq k u(x) \cot _{b}(u(x)),
$$

for all $x \in B_{g}\left(\xi, r_{0}\right)$, and for all $T \in \operatorname{Gr}\left(k, T_{\xi} M\right)$.

Remark 2.2.3. Notice that we only use the estimate from above of the Theorem 2.2.6, which will be enough for our purposes, but if we instead use the estimate from below. To do so, we must assume that in Lemma 2.2.1, that $b^{-} \leq \operatorname{Seg}_{g} \leq b^{+}$, where $b^{-} \leq 0$ and $b^{+} \geq 0$ and $s n_{\frac{1}{2}\left(b^{-}+b^{+}\right)}>0$ on $\eta$, the geodesic along $J$ is defined in the proof of Theorem 2.2.6, and we will get, that for all $x \in B_{b}\left(\xi, r_{0}\right)$ 
and $T \in \operatorname{Gr}\left(k, T_{\xi} M\right)$

$$
k u(x) \cot _{\left(b^{+}\right)}(u(x)) \leq \operatorname{div}_{T}(u \nabla u) \leq k u(x) \cot _{\left(-b^{-}\right)}(u(x)) .
$$




\section{Chapter 3}

\section{Intrinsic Riemannian Theory of General Varifolds}

\subsection{General Varifolds}

Now we introduce the notations and concepts relative to varifolds that we need to make a Riemannian intrinsic theory of varifolds. In this respect we closely follow [All72], [Sim83], [Lel12], [NOA18]. In what follows $V$ will always denote a varifold and $d v_{g}$ the Riemannian measure of $\left(M^{n}, g\right)$.

Definition 3.1.1. For any $k, n \in \mathbb{N}, n \geq 2,1 \leq k \leq n-1$, let $M^{n}$ a $n$-dimensional manifold. We say that $V$ is a $k$-dimensional varifold in $M$, if $V$ is a nonnegative, real extended valued, Radon measure on $G_{k}\left(M^{n}\right)$ the Grassmannian manifold whose underlying set is the union of the sets $\operatorname{Gr}\left(k, T_{x} M\right)$, where $\operatorname{Gr}\left(k, T_{x} M\right)$ denotes the set of $k$-dimensional subspaces of $T_{x} M^{n}$, as $x$ varies on $M^{n}$ (compare with section 2.6 of [All72]). For every $k \in\{1, \ldots, n-1\}$, we define $\mathbf{V}_{k}\left(M^{n}\right)$ to be the space of all $k$-dimensional varifolds on $M^{n}$ endowed with the weak topology induced by $C_{c}^{0}\left(G_{k}\left(M^{n}\right)\right)$, say the space of continuous compactly supported functions on $G_{k}\left(M^{n}\right)$ endowed with the compact open topology.

Definition 3.1.2. Let $V \in \mathbf{V}_{k}\left(M^{n}\right)$, g is a Riemannian metric on $M^{n}$, we say that the nonnegative Radon measure on $M^{n},\|V\|$ is the weight of $V$, if $\|V\|=\pi_{\#}(V)$, here $\pi$ indicates the natural fiber bundle projection $\pi: G_{k}\left(M^{n}\right) \rightarrow M^{n}, \pi:(x, S) \mapsto x$, for every $(x, S) \in G_{k}\left(M^{n}\right), x \in M^{n}$, $S \in \operatorname{Gr}\left(k, T_{x} M\right)$,

$$
\|V\|(A):=V\left(\pi^{-1}(A)\right)
$$

Remark 3.1.1. Recall that $\pi$ is a proper map because the fibers of the fiber bundle $G_{k}\left(M^{n}\right) \stackrel{\pi}{\rightarrow} M^{n}$ are compact.

As the reader has noticed, an abstract varifold can be a quite strange object, because it is hard to work with Borel sets on $G_{k}\left(M^{n}\right)$ in an operative way (here operative way has to be understate in a sense to be specified later in this section); but in the sequel, we also define the weight of $V$ which is a Radon measure on $\Sigma$ obtained from $V$ by ignoring the fiber variable. The next theorem illustrates how to "simplify" a varifold in an operative way. This result is a direct application of a well known disintegration Theorem, which can be found in [AFP00] Theorem 2.28. However the following is an adaptation of it into the context of our intrinsic varifolds.

Theorem 3.1.1 (Disintegration Theorem for Varifolds). Let $\left(M^{n}, g\right)$ a n-dimensional Riemannian manifold, let $V \in \mathbf{V}_{k}\left(M^{n}\right)$, and $\pi: G_{k}\left(M^{n}\right) \rightarrow M$ be the canonical projection onto $M^{n}$. Then there exists a family of Radon measures $\left\{\pi_{x}\right\}_{x \in M^{n}}$ such that, the map $x \mapsto \pi_{x}$ is $\|V\|$-measurable and the 
following relations are satisfied

$$
\begin{aligned}
& \pi_{x}(B):=\lim _{r \downarrow 0} \frac{V\left(B_{g}(x, r) \times B\right)}{\|V\|(B(x, r))}, \quad \text { for all } B \in \mathcal{B}\left(G r\left(k, T_{x} M^{n}\right)\right), \\
& \pi_{x}\left(G r\left(k, T_{x} M^{n}\right) \backslash\left\{S: S \subset \operatorname{Tan}\left(M^{n}, x\right)\right\}\right)=0, \text { and } \pi_{x}\left(G r\left(k, T_{x} M^{n}\right)\right)=1, \\
& f(x, \cdot) \in L^{1}\left(G r\left(k, T_{x} M^{n}\right), \pi_{x}\right), \text { for }\|V\|-\text { a.e. } x \in M^{n}, \\
& x \mapsto \int_{G r\left(k, T_{x} M^{n}\right)} f(x, S) d \pi_{x}(S) \in L^{1}\left(M^{n},\|V\|\right), \text { is }\|V\|-\text { measurable, } \\
& \int_{G_{k}\left(M^{n}\right)} f(x, S) d V(x, T)=\int_{M^{n}}\left(\int_{G r\left(k, T_{x} M^{n}\right)} f(x, S) d \pi_{x}(S)\right) d\|V\|(x),
\end{aligned}
$$

for any $f \in L^{1}\left(G_{k}\left(M^{n}\right), V\right)$. Moreover, if $\pi_{x}^{\prime}$ is any other $\|V\|$-measurable map satisfying (3.4) and (3.5) for every bounded Borel function with compact support and such that $x \mapsto \pi_{x}^{\prime}\left(G r\left(k, T_{x} M^{n}\right)\right) \in$ $L^{1}\left(M^{n},\|V\|\right)$, then $\pi_{x}=\pi_{x}^{\prime}$ for $\|V\|$-a.e. $x \in M^{n}$.

Corollary 3.1.1.1. Let $V \in \mathbf{V}_{k}\left(M^{n}\right)$, with the same notation of the Theorem 3.1.1, the equality

$$
V=\|V\| \otimes \pi_{x}
$$

holds.

\subsection{The First Variation of a Varifold}

According to [All72], we associate to each varifold $V$ a vector valued distribution, which depends on the metric $g$ of the ambient space, called the first variation of $V$. If this first variation is (as Radon measure measure) absolute continuous with respect to weight measure $\|V\|$ in analogy with the smooth case we define the generalized mean curvature vector $H_{g}$.

Definition 3.2.1. Let $\left(M^{n}, g\right)$ a n-dimensional Riemannian manifold with Levi-Civita connection $\nabla, \mathfrak{X}_{c}^{1}(M)$ the set of differentiable vector fields on $M$ and $V \in \mathbf{V}_{k}(M)$ a $k$-dimensional varifold $(k \leq n)$. We define the first variation of $V$ along the vector field $X \in \mathfrak{X}_{c}^{1}(M)$ as

$$
\delta V(X):=\int_{G_{k}(M)}\left\langle\nabla X(x) \circ p_{T}, p_{T}\right\rangle_{g} d V(x, T),
$$

where the inner product in the integrand is the one defined in $\operatorname{Hom}\left(T_{x} M, T_{x} M\right)$, and $\nabla X: \mathfrak{X}(M) \rightarrow$ $\mathfrak{X}(M)$ such that $\nabla X(Y):=\nabla_{Y} X$.

Let $\left\{\tau_{1}, \ldots, \tau_{k}\right\}$ an orthonormal basis of $T \in \operatorname{Gr}\left(k, T_{x} M\right)$ for $x$ given, and $\left\{\tau_{1}, \ldots, \tau_{k}, \tau_{k+1}, \ldots \tau_{n}\right\}$ the completion to an orthonormal basis for $T_{x} M$, then for given $X \in \mathfrak{X}_{c}^{1}(M)$,

$$
\begin{aligned}
\delta V(X) & =\int_{G_{k}(M)} \operatorname{trace}\left(\left(\nabla X(x) \circ p_{T}\right)^{*} \circ p_{T}\right) d V(x, T) \\
& =\int_{G_{k}(M)} \sum_{i=1}^{k}\left\langle\tau_{i}, \nabla_{\tau_{i}} X(x)\right\rangle_{g} d V(x, T)
\end{aligned}
$$

so, we have that, for all $X \in \mathfrak{X}_{c}^{1}(M)$, the definition (3.2.1) is equivalent to say,

$$
\delta V(X)=\int_{G_{k}(M)} \operatorname{div}_{T} X(x) d V(x, T),
$$

where, for given $x$, and $\left\{\tau_{1}, \ldots, \tau_{k}\right\}$ an orthonormal basis of a fixed $T \in \operatorname{Gr}\left(k, T_{x} M\right)$,

$$
\operatorname{div}_{T} X(x)=\sum_{i=1}^{k}\left\langle\tau_{i}, \nabla_{\tau_{i}} X(x)\right\rangle_{g} .
$$


Then (3.6) give us formula with more geometric meaning than the merely definition, on the other hand, by analogy with the smooth case, we desire to related in some way the first variation of a varifold with a "mean curvature" vector, to do so, let us first analyze the total variation of the first variation.

Definition 3.2.2. Let $V \in \mathbf{V}_{k}(M)$ with $(M, g)$ a n-dimensional Riemannian manifold, we say that $V$ has locally bounded first variation in $U \subset M$ open set, if for all $W \subset \subset U$ open set, there exists a constant $C:=C(W)$ such that

$$
|\delta V(X)| \leq C\|X\|_{L^{\infty}(W, V)}
$$

for all $X \in \mathfrak{X}_{c}^{0}(W)$.

Remark 3.2.1. Notice that the definition of bounded first variation is valid for each $X \in \mathfrak{X}_{c}^{0}(M)$ while the first variation, $\delta V$, is defined on $\mathfrak{X}_{c}^{1}(M)$. However, defining an extension $\widetilde{\delta V}: \mathfrak{X}_{c}^{0}(M) \rightarrow \mathbb{R}$ of $\delta V$ as

$$
\widetilde{\delta V}(X):=\lim _{\varepsilon \downarrow 0} \delta V\left(X_{\varepsilon}\right)
$$

where $\left(X^{1}, \ldots, X^{n}\right)=X \in \mathfrak{X}_{c}^{0}(M)$, and $X_{\varepsilon} \in \mathfrak{X}_{c}^{1}(M)$ is a "C $C^{1}$ approximation" of $X$ defined as follows:

Let $\left\{\left(\Phi_{i}, U_{i}\right)\right\}_{i \in \Lambda}$ an atlas of $M$, since we are interested in compactly vector fields we can choose $\Lambda=\{1, \ldots, N\}$ such that $\left\{U_{i}\right\}_{i=1}^{N}$ is an open covering for $\operatorname{spt} X$, and consider $\{\psi\}_{i=1}^{\ell}$ a partition of unity subordinate to $\left\{U_{i}\right\}_{i=1}^{N}$, then

$$
X_{\varepsilon}(p):=\sum_{i=1}^{\ell}\left(\sum_{j=1}^{N}\left(\left.\psi_{j} X^{i}\right|_{U_{i}} \star \varphi^{\varepsilon}\right)(p)\right) \frac{\partial}{\partial x_{i}},
$$

where $\varphi^{\varepsilon}$ is an standard approximation of the identity in $\mathbb{R}^{n}$, and $\star$ denotes the usual convolution. Clearly $X_{\varepsilon}$ is independent of the choice of charts and defined on $\operatorname{spt} X$. Furthermore by standard theory of convolutions, we know that,

$$
\left.\left.\psi_{j} X^{i}\right|_{U_{i}} \star \varphi^{\varepsilon} \rightarrow \psi_{j} X^{i}\right|_{U_{j}}
$$

uniformly in compacts, when $\varepsilon \rightarrow 0$. Hence

$$
\left\|X-X_{\varepsilon}\right\|_{L^{\infty}} \rightarrow 0, \text { when } \varepsilon \rightarrow 0
$$

and $X_{\varepsilon} \in \mathfrak{X}_{c}^{1}(M)$.

On the other hand, given $X_{\varepsilon}^{1}, X_{\varepsilon}^{2} \in \mathfrak{X}_{c}^{1}(M)$ as above such that $X_{\varepsilon}^{1} \rightarrow X$ and $X_{\varepsilon}^{1} \rightarrow X$, assume that $V$ has bounded first variation on $M$, then

$$
\left|\delta V\left(X_{\varepsilon}^{1}-X_{\varepsilon}^{2}\right)\right| \leq C\left\|X_{\varepsilon}^{1}-X_{\varepsilon}^{1}\right\|_{L^{\infty}} \rightarrow 0 \quad \text { when } \varepsilon \rightarrow 0,
$$

which implies that

$$
\widetilde{\delta V}: \mathfrak{X}_{c}^{0}(M) \rightarrow \mathbb{R}
$$

is well defined and $\widetilde{\delta V} \equiv \delta V$ on $\mathfrak{X}_{c}^{1}(M)$. By abuse os notation we will identify $\widetilde{\delta V}$ with $\delta V$.

Proposition 3.2.1. Let $V \in \mathbf{V}_{k}\left(M^{n}\right)$ with locally bounded first variation in $\left(M^{n}, g\right)$. Then the total variation $\|\delta V\|$ is a Radon measure. Furthermore, there exist a $\|V\|$-measurable function $H_{g}$ : $M \rightarrow T M$, and $Z \subset M$ with $\|V\|(Z)=0$, such that

$$
\delta V(X)=-\int_{M^{n}}\left\langle X, H_{g}\right\rangle_{g} d\|V\|+\int_{M^{n}}\langle X, \nu\rangle_{g} d\|\delta V\|_{\text {sing }},
$$


where $\nu$ is a $\|\delta V\|$-measurable function with $|\nu(x)|_{g}=1$, and $\|\delta V\|_{\text {sing }}=\|\delta V\|\left\llcorner Z\right.$. We call $H_{g}$ the generalized mean curvature vector of $V$.

Proof. Since by hypothesis $V$ has locally bounded first variation, for all open set $W \subset \subset M$

$$
\|\delta V\|(W)=\sup \left\{|\delta V(X)|: X \in \mathfrak{X}_{c}^{0}(W),|X| \leq 1\right\} \leq C<\infty .
$$

Then by the Riesz Representation Theorem for $\mathfrak{X}_{c}^{0}(M)$ (where we are identifying $\widetilde{\delta V}$ with $\delta V$ ) there exists $\nu: M \rightarrow T M\|\delta V\|$-measurable function with $|\nu(x)|_{g}=1\|\delta V\|$-a.e. and for all $X \in \mathfrak{X}_{c}^{0}(M)$

$$
\delta V(X)=\int_{M}\langle X, \nu\rangle_{g} d\|\delta V\|,
$$

and $\|\delta V\|$ is a Radon measure. This last assertion prove the first part of the theorem as in the Euclidean case. To prove the second part of the theorem we need to make use of the fact that the Vitali symmetry property holds for every closed metric balls of $\left(M^{n}, g\right)$. This guarantees that we can apply the Lebesgue Differentiation Theorem to space $B_{g}\left(\xi, i n j_{M^{n}, g}(\xi)\right)$ because $\|V\|$ is a regular enough Radon measure (see Remark 2.1.1). Thus, by the Lebesgue Differentiation Theorem we get

$$
D_{\|V\|}\|\delta V\|=\lim _{\rho \downarrow 0} \frac{\|\delta V\|\left(B_{g}(x, \rho)\right)}{\|V\|\left(B_{g}(x, \rho)\right)}
$$

exists $\|V\|$-a.e and (writing $-H_{g}(x):=D_{\|V\|}\|\delta V\|(x) \nu(x)$ ) we are lead to the following formula

$$
\delta V(X)=-\int_{M}\left\langle X(x), H_{g}(x)\right\rangle_{g} d\|V\|(x)+\int\langle X(x), \nu(x)\rangle_{g} d\|\delta V\|_{\text {sing }}
$$

where $\|\delta V\|_{\text {sing }}=\|\delta V\|\left\llcorner\left\{x: D_{\|V\|}\|\delta V\|=+\infty\right\}\right.$.

Remark 3.2.2. Let $\left(M^{n}, g\right)$ be an n-dimensional Riemannian manifold, let $V \in \mathbf{V}_{k}(M)$ such that for some $C>0, p>k, 0<\rho<i n j_{\xi}(M, g)$ for fixed $\xi \in M$

$$
\delta V(X) \leq C\left(\int_{B_{g}(\xi, \rho)}|X|^{\frac{q}{q-1}} d\|V\|\right)^{\frac{q-1}{q}} .
$$

Then, by Hölder inequality we have that

$$
|\delta V(X)| \leq C_{1}\|X\|_{L^{\infty}\left(\|V\|, \operatorname{spt} X \cap B_{g}(\xi, \rho)\right)},
$$

where $C_{1}(\xi, \rho):=C\left(\|V\|\right.$ (spt $\left.\left.X \cap B_{g}(\xi, \rho)\right)\right)^{\frac{q-1}{q}}$. Then $V$ has locally bounded first variation, furthermore, $\|\delta V\|$ is a Radon measure on $M$. Roughly speaking, if we assume that $\operatorname{spt} X \subset A$, for $A$ a given $\|V\|$-negligible set, and take the supremum over all such $X$ 's with $\|X\|_{L^{\infty}(\|V\|)}=1$ then by (3.7) we get that

$$
\|\delta V\|(A) \leq C(\|V\|(A))^{\frac{q-1}{q}}=0,
$$

which implies that $\|\delta V\| \ll\|V\|$. In fact, technically to prove the preceding equation we need to be more carefull, i.e., we need to take a sequence of open sets $A \subset U_{j} \subseteq B_{g}(\xi, \rho), \forall j$ such that $U_{j} \rightarrow A$, then using (3.7) we have that

$$
\delta V(X) \leq C\left(\int_{U_{j}}|X|^{\frac{q}{q-1}} d\|V\|\right)^{\frac{q-1}{q}}, \forall X \in \mathfrak{X}_{c}^{1}\left(U_{j}\right) .
$$

Then taking limits, it easy to check that

$$
\|\delta V\|(A)=\lim _{j \rightarrow \infty}\|\delta V\|\left(U_{j}\right) \leq \lim _{j \rightarrow \infty} C\left(\|V\|\left(U_{j}\right)\right)^{\frac{q-1}{q}}=0 .
$$


Hence, the Radon-Nikodym Theorem gives the existence of a $L^{1}\left(M^{n},\|V\|\right)$ function $H_{g}$ such that

$$
\delta V(X)=\int_{G_{k}(M)} \operatorname{div}_{S} X(y) d V(y, T)=-\int_{M}\left\langle H_{g}(y), X(y)\right\rangle_{g} d\|V\|(y) .
$$

On the other hand, (3.7) means that $\delta V(X)$ is a bounded linear functional on $L_{l o c}^{q}(\Gamma(T M),\|V\|)$ of the $L_{\text {loc }}^{q}$ vector fields on $M$ with respect to $\|V\|$. So by well known theorems of measure theory (compare Theorem 6.16 of [Rud87]) stating that the topological dual of $L^{q}$ is $L^{p}$, i.e., $\left(L^{q}\right)^{*}=L^{p}$ we have that there exists a function $f \in L^{p}$ such that $\delta V(X)=\int_{B_{g}(\xi, \rho)}\langle f, X\rangle_{g}$ and the best constant $C>0$ in (3.7) is given by $\|f\|_{L^{p}}$. Combining (3.7) and (3.8) we get that $f=H_{g}$ and so that $H_{g} \in L_{l o c}^{p}(\Gamma(T M),\|V\|)$. Finally, notice that, for all $X \in \mathfrak{X}_{c}^{1}(M)$ (again using the Hölder inequality)

$$
\delta V(X) \leq\left\|H_{g}\right\|_{L^{p}(\|V\|, M)}\|X\|_{L^{\frac{q}{q-1}(\|V\|, M)}} .
$$

At this stage we have proved that (3.7) implies that $V$ has locally bounded first variation, no singular part and the generalized mean curvature vector field $H_{g}$, satisfies an $L^{p}$ condition.

Definition 3.2.3 (Allard's Conditions). Given $V$ a $k$-dimensional varifold, we say that $V$ satisfies an Allard's type condition for the generalized mean curvature, if $V$ satisfies (3.7). From now on, denoted as $(A C)$.

This kind of conditions will play a key role in the section (4.1) when we study the monotonicity behaviour of the density ratio. 


\section{Chapter 4}

\section{Monotonicity and Consequences}

The aim of this section is to obtain information about $V$ from their first variation $\delta V(X)$, for an appropriate choice of $X \in \mathfrak{X}_{0}^{1}(U)$. This kind of result is known as "Monotonicity Formula", and is the step zero in any interior regularity theory.

\subsection{Weighted Monotonicity Formulae for Abstrac Varifolds}

Let $(M, g)$ a Riemannian manifold with Levi-Civita connection $\nabla$ and $V \in \mathbf{V}_{k}(M)$ satisfying $(A C)$, fix $\xi \in M$ and assume $i n j_{\xi}(M, g)>0$. Choose $r_{0}>0$ such that $r_{0}<i n j_{\xi}(M, g)$, and, for fixed $\varepsilon>0$ let $\gamma_{\varepsilon} \in C_{c}^{1}(]-\infty, 1[)$, such that

$$
\gamma_{\varepsilon}(y):=\left\{\begin{array}{ll}
1 & \text { if } y \leq \varepsilon, \\
0 & \text { if } y>1,
\end{array} \quad \text { and } \quad \gamma_{\varepsilon}^{\prime}(y)<0 \text { if } \varepsilon<y<1,\right.
$$

Then we can consider the radial perturbation

$$
\tilde{X}_{s, \varepsilon}(x)=\left(\gamma_{\varepsilon}\left(\frac{u(x)}{s}\right)(u \nabla u)\right)(x), \quad \text { for } 0<|s|<r_{0},
$$

where $u(x)=r_{\xi}(x)=\operatorname{dist}_{(M, g)}(x, \xi)$. Now, let $y \in M$ given, and $T \in \operatorname{Gr}\left(k, T_{y} M\right)$. Let $\left\{e_{1}^{T}, \ldots, e_{k}^{T}\right\}$ an orthonormal basis for $S$, then

$$
\begin{aligned}
\operatorname{div}_{T} \tilde{X}_{s, \varepsilon} & =\sum_{i=1}^{k}\left\langle e_{i}^{T}, \nabla_{e_{i}^{T}} \gamma_{\epsilon}\left(\frac{u}{s}\right)(u \nabla u)\right\rangle_{g} \\
& =\gamma_{\varepsilon}^{\prime}\left(\frac{u}{s}\right) \frac{u}{s} \sum_{i=1}^{k}\left\langle e_{i}^{T}, \nabla u\right\rangle_{g}^{2}+\gamma_{\epsilon}\left(\frac{u}{s}\right) \sum_{i=1}^{k}\left\langle e_{i}^{T}, \nabla_{e_{i}^{T}}(u \nabla u)\right\rangle_{g} \\
& =\gamma_{\epsilon}\left(\frac{u}{s}\right) \operatorname{div}_{T}(u \nabla u)+\gamma_{\epsilon}^{\prime}\left(\frac{u}{s}\right) \frac{u}{s}\left|\nabla^{\top} u\right|_{g}^{2},
\end{aligned}
$$

where $\nabla^{T} u$ is the orthogonal projection of $\nabla u$ onto $S$. Let $\nabla^{T^{\perp}} u$ denote the orthogonal projection of $\nabla u$ onto $T^{\perp}$, then,

$$
\left|\nabla^{T} u\right|_{g}^{2}+\left|\nabla^{T^{\perp}} u\right|_{g}^{2}=|\nabla u|_{g}^{2}=1,
$$

since $u$ is a distance function. Therefore

$$
\operatorname{div}_{T} \tilde{X}_{s, \varepsilon}=\gamma_{\epsilon}\left(\frac{u}{s}\right) \operatorname{div}_{T}(u \nabla u)+\gamma_{\epsilon}^{\prime}\left(\frac{u}{s}\right) \frac{u}{s}-\gamma_{\epsilon}^{\prime}\left(\frac{u}{s}\right) \frac{u}{s}\left|\nabla^{S^{\perp}} u\right|_{g}^{2}
$$

Although this choice is enough to get many useful information, let us consider a general case, which will be used in the sequel. Let $h \in C^{1}(U)$ a non-negative function, and consider

$$
X_{s, \varepsilon}(y):=h(y) \tilde{X}_{s}(y)=h(y)\left(\gamma_{\varepsilon}\left(\frac{u(y)}{s}\right)(u \nabla u)\right)(y), \quad \text { for } 0<|s|<r_{0} .
$$


Then, if $\left\{e_{1}^{S}, \ldots, e_{k}^{T}\right\}$ is as above,

$$
\begin{aligned}
\operatorname{div}_{T} X_{s, \varepsilon}(y) & =\sum_{i=1}^{k}\left\langle e_{i}^{T}, \nabla_{e_{i}^{T}} h(y) \tilde{X}_{s}(y)\right\rangle_{g} \\
& =h(y) \sum_{i=1}^{k}\left\langle e_{i}^{T}, \nabla_{e_{i}^{T}} \tilde{X}_{s}(y)\right\rangle_{g}+\sum_{i=1}^{k}\left\langle\left(\nabla_{e_{i}^{T}} h(y)\right) e_{i}^{T}, \tilde{X}_{s}(y)\right\rangle_{g} \\
& =h(y) \operatorname{div}_{T} \tilde{X}_{s, \varepsilon}+\left\langle\nabla^{T} h(y), \tilde{X}_{s}(y)\right\rangle_{g} .
\end{aligned}
$$

By the definition of the first variation, we know that

$$
\begin{aligned}
\delta V\left(X_{s, \varepsilon}\right): & =\int_{G_{k}(U)} \operatorname{div}_{T} X_{s, \varepsilon}(y) d V(y, T) \\
& =\int_{G_{k}(U)} h(y) \operatorname{div}_{T} \tilde{X}_{s}(y) d V(y, T)+\int_{G_{k}(U)}\left\langle\nabla^{T} h(y), \tilde{X}_{s}(y)\right\rangle_{g} d V(y, T) .
\end{aligned}
$$

Then, replacing (4.1) in (4.2) and the information above, we have

$$
\begin{aligned}
\delta V\left(X_{s, \varepsilon}\right) & =\int_{G_{k}(U)} h(y) \gamma_{\varepsilon}\left(\frac{u(y)}{s}\right) \operatorname{div}_{T}(u \nabla u)(y) d V(y, T) \\
& +\int_{G_{k}(U)} \frac{h(y) u(y)}{s} \gamma_{\varepsilon}^{\prime}\left(\frac{u(y)}{s}\right) d V(y, T) \\
& -\int_{G_{k}(U)} \frac{h(y) u(y)}{s} \gamma_{\varepsilon}^{\prime}\left(\frac{u(y)}{s}\right)\left|\nabla^{T^{\perp}} u\right|_{g}^{2} d V(y, T) \\
& +\int_{G_{k}(U)} \gamma_{\varepsilon}\left(\frac{u(y)}{s}\right)\left\langle\nabla^{T} h(y),(u \nabla u)(y)\right\rangle_{g} d V(y, T)
\end{aligned}
$$

If we compare (4.3) with the Euclidean case (see Cf. [Sim83] (4.14) and (4.24)) we note the lack of a dimensional term, then, in order to have an intrinsic result, we need to compare it in some way with the Euclidean case. To do this we use the Rauch's comparison theorem, applied as in Lemma 3.6 of [HS74], which states:

Lemma 4.1.1. Let $(M, g)$ be a complete Riemannian manifold, with Levi-Civita connection $\nabla$, let $b \in \mathbb{R}$ such that $S e c_{g} \leq b$, assume $b r_{0}<\pi$. Then

$$
\operatorname{div}_{T}(u \nabla u)(x) \geq k u(x) \cot _{b}(u(x)),
$$

for all $x \in B_{g}\left(\xi, r_{0}\right)$.

Remark 4.1.1. Notice that under the conditions of Lemma 4.1.1 we can compare the divergence of the radial field $u \nabla u$ in the ambient manifold $M^{n}$ with the same quantity calculated in a space-form, so, is natural to guess that this is the framework to get some "monotonicity" behaviour (inspired in the results from the euclidean case). Furthermore, in view of Remark 2.2.1 above, we expect to recovery in some sense the euclidean case, this is why from now we will be in the setting of the Lemma 4.1.1, i.e.

Definition 4.1.1 ( Bounded Geometry). We say that a complete Riemannian manifold $\left(M^{n}, g\right)$ has bounded geometry (or satisfy $(B G)$ ), if $S_{e c}<b$ for some constant $b \in \mathbb{R}$ and for every $\xi \in M$ there is $r_{0}$ such that $0<r_{0}<i n j_{\xi}(M, g)$ and $r_{0} b<\pi$. 
In view of the Remarks 2.2.1 and 4.1.1 we can define $c(s):=c(s, b)$ as:

$$
c(s)= \begin{cases}s \sqrt{b} \cot (\sqrt{b} s) & b>0 \\ 0 & b \leq 0\end{cases}
$$

where $b \in \mathbb{R}$. Then, by the Lemma 4.1.1,

$$
\operatorname{div}_{T}((u \nabla u)(x)) \geq k c(u(x), b),
$$

for all $x \in B_{g}\left(\xi, r_{0}\right)$, furthermore we can assume $r_{0} \cot _{b}\left(r_{0}\right)>0$, since we are interested in small geodesic balls around $\xi$.

Then, substituting in (4.3) we have

$$
\begin{aligned}
\delta V\left(X_{s, \varepsilon}\right) & \geq \int_{G_{k}(M)} k \operatorname{ch}(y) \gamma_{\varepsilon}\left(\frac{u(y)}{s}\right) d V(y, T) \\
& +\int_{G_{k}(M)} \gamma_{\varepsilon}\left(\frac{u(y)}{s}\right)\left\langle\nabla^{T} h(y),(u \nabla u)(y)\right\rangle_{g} d V(y, T) \\
& +\int_{G_{k}(M)} \frac{h(y) u(y)}{s} \gamma_{\varepsilon}^{\prime}\left(\frac{u(y)}{s}\right) d V(y, T) \\
& -\int_{G_{k}(M)} \frac{h(y) u(y)}{s} \gamma_{\varepsilon}^{\prime}\left(\frac{u(y)}{s}\right)\left|\nabla^{T^{\perp}} u\right|_{g}^{2} d V(y, T) .
\end{aligned}
$$

On the other hand, $\operatorname{since}(s)$ is decrrasing, $c(u(x)) \geq c(s) \geq c\left(r_{0}\right)$ whenever $0 \leq u(x) \leq s \leq$ $r_{0}=r_{0}(b)$. Hence,

$$
\begin{aligned}
-\int_{G_{k}(M)}\left[h k \gamma_{\varepsilon}\left(\frac{u}{s}\right)+\frac{h u}{s} \gamma_{\varepsilon}^{\prime}\left(\frac{u}{s}\right)\right] d V & \geq \int_{G_{k}(M)}-\frac{h u}{s} \gamma_{\varepsilon}^{\prime}\left(\frac{u}{s}\right)\left|\nabla^{T^{\perp}} u\right|_{g}^{2} d V \\
& +\int_{G_{k}(M)} \gamma_{\varepsilon}\left(\frac{u}{s}\right)\left\langle\nabla^{T} h,(u \nabla u)\right\rangle_{g} d V \\
& +(c(s)-1) \int_{G_{k}(M)} h k \gamma_{\varepsilon}\left(\frac{u}{s}\right) d V \\
& -\delta V\left(X_{s, \varepsilon}\right),
\end{aligned}
$$

now, dividing by $s^{k+1}$,

$$
\begin{aligned}
-\int_{G_{k}(M)} h(y) I(s) d V(y, T) & \geq \int_{G_{k}(M)} \frac{h(y)}{s^{k}} \frac{\partial}{\partial s}\left(\gamma_{\varepsilon}\left(\frac{u(y)}{s}\right)\right)\left|\nabla^{S^{\perp}} u\right|_{g}^{2} d V(y, T) \\
& +\frac{1}{s^{k+1}}\left(\int_{G_{k}(M)} \gamma_{\varepsilon}\left(\frac{u(y)}{s}\right)\left\langle\nabla^{T} h(y),(u \nabla u)(y)\right\rangle_{g} d V(y, T)\right) \\
& +\frac{(c(s)-1)}{s} \frac{k}{s^{k}} \int_{G_{k}(M)} h(y) \gamma_{\varepsilon}\left(\frac{u(y)}{s}\right) d V(y, T) \\
& -\frac{\delta V\left(X_{s, \varepsilon}\right)}{s^{k+1}},
\end{aligned}
$$


where

$$
\begin{aligned}
I(s): & =\frac{k}{s^{k+1}} \gamma_{\varepsilon}\left(\frac{u(y)}{s}\right)+\frac{u(y)}{s^{k+2}} \gamma_{\varepsilon}^{\prime}\left(\frac{u(y)}{s}\right) \\
& =-\frac{d}{d s}\left(\frac{1}{s^{k}}\right) \gamma_{\varepsilon}\left(\frac{u(y)}{s}\right)-\frac{1}{s^{k}} \frac{d}{d s}\left(\gamma_{\varepsilon}^{\prime}\left(\frac{u(y)}{s}\right)\right) \\
& =-\frac{d}{d s}\left(\frac{1}{s^{k}} \gamma_{\varepsilon}\left(\frac{u(y)}{s}\right)\right) .
\end{aligned}
$$

Differentiating under the sign of integral we have

$$
\begin{aligned}
\frac{d}{d s}\left(\frac{1}{s^{k}} \int_{G_{k}(M)} h \gamma_{\varepsilon}\left(\frac{u}{s}\right) d V\right) & \geq \int_{G_{k}(M)} \frac{h}{s^{k}} \frac{\partial}{\partial s}\left(\gamma_{\varepsilon}\left(\frac{u}{s}\right)\right)\left|\nabla^{T^{\perp}} u\right|_{g}^{2} d V \\
& +\frac{1}{s^{k+1}}\left(\int_{G_{k}(M)} \gamma_{\varepsilon}\left(\frac{u}{s}\right)\left\langle\nabla^{T} h,(u \nabla u)\right\rangle_{g} d V\right) \\
& +\frac{(c(s)-1)}{s} \frac{k}{s^{k}} \int_{G_{k}(M)} h \gamma_{\varepsilon}\left(\frac{u}{s}\right) d V \\
& -\frac{\delta V\left(X_{s, \varepsilon}\right)}{s^{k+1}} .
\end{aligned}
$$

Now, by Theorem 3.1.1,

$$
\int_{G_{k}(M)} h(y) \gamma_{\varepsilon}\left(\frac{u(y)}{s}\right) d V(y, T)=\int_{M} h(y) \gamma_{\varepsilon}\left(\frac{u(y)}{s}\right) d\|V\|(y)
$$

and

$$
\int_{G_{k}(M)} h(y) k \gamma_{\varepsilon}\left(\frac{u(y)}{s}\right) d V(y, T)=\int_{M} h(y) k \gamma_{\varepsilon}\left(\frac{u(y)}{s}\right) d\|V\|(y) .
$$

Thus,

$$
\begin{aligned}
\frac{d}{d s}\left(\frac{1}{s^{k}} \int_{M} h \gamma_{\varepsilon}\left(\frac{u}{s}\right) d\|V\|\right) & \geq \int_{G_{k}(M)} \frac{h}{s^{k}} \frac{\partial}{\partial s}\left(\gamma_{\varepsilon}\left(\frac{u}{s}\right)\right)\left|\nabla^{T^{\perp}} u\right|_{g}^{2} d V \\
& +\frac{1}{s^{k+1}}\left(\int_{G_{k}(M)} \gamma_{\varepsilon}\left(\frac{u}{s}\right)\left\langle\nabla^{T} h,(u \nabla u)\right\rangle_{g} d V\right) \\
& +\frac{(c(s)-1)}{s} \frac{k}{s^{k}} \int_{G_{k}(M)} h \gamma_{\varepsilon}\left(\frac{u}{s}\right) d\|V\| \\
& -\frac{\delta V\left(X_{s, \varepsilon}\right)}{s^{k+1}} .
\end{aligned}
$$

Remark 4.1.2. Notice that, by the Remark 2.2.1 the behavior of $c(s):=c(s, b)$, we have that

$$
\frac{(c(s)-1)}{s}=-O(s)=O(s), \quad \text { as } s \rightarrow 0^{+}
$$

furthermore, this is decreasing, and

$$
\frac{(c(s)-1)}{s} \geq \frac{\left(c\left(r_{0}\right)-1\right)}{r_{0}}:=c_{1}^{*}
$$

Theorem 4.1.2 (Fundamental Weighted Monotonicity Inequality). Let $\left(M^{n}, g\right)$ be a complete Riemannian Manifold with Levi-Civita connection $\nabla$, satisfying $(B G)$, and $r_{0} \cot _{b}\left(r_{0}\right)>0$. Let $V \in \mathbf{V}_{k}\left(M^{n}\right)$ satisfying $(A C)$, then for any $0<s<r_{0}$. There exists a constant $\left.c=c(s, b) \in\right] 0,1[$ 
such that, if we set $u(x)=r_{\xi}(x)=\operatorname{dist}_{(M, g)}(x, \xi)$ we have for all $0<s<r_{0}$

$$
\begin{aligned}
\frac{d}{d s}\left(\frac{1}{s^{k}} \int_{B_{g}(\xi, s)} h d\|V\|\right) & \geq \frac{1}{s^{k+1}}\left(\int_{G_{k}\left(B_{g}(\xi, s)\right)}\left\langle\nabla^{T} h,(u \nabla u)\right\rangle_{g} d V\right) \\
& +\frac{(c(s)-1)}{s} \frac{k}{s^{k}} \int_{B_{g}(\xi, s)} h d\|V\| \\
& +\frac{1}{s^{k+1}} \int_{B_{g}(\xi, s)}\langle H, h(u \nabla u)\rangle_{g} d\|V\| \\
& +\frac{d}{d s} J(s),
\end{aligned}
$$

where

$$
J(s):=\int_{G_{k}\left(B_{g}(\xi, s)\right)} h(y) \frac{\left|\nabla^{T^{\perp}} u\right|_{g}^{2}}{r_{\xi}^{k}} d V(y, T) .
$$

Proof. Let $\left\{\varepsilon_{j}\right\}_{j \in \mathbb{N}}$ a sequence, such that $\varepsilon_{j} \uparrow 1$ when $j \rightarrow \infty$ and $\left\{\gamma_{\varepsilon_{j}}\right\} \subset C_{0}^{1}(]-\infty, 1[)$ a sequence of mollifiers, such that $\gamma_{\varepsilon_{j}} \rightarrow \chi_{]-\infty, 1}$, pointwise from below. Considering $X_{s, \varepsilon_{j}}$ as in (4.1) by the previous reasoning (see (4.4)) we have for every $j \in \mathbb{N}$

$$
\begin{aligned}
\frac{d}{d s}\left(\frac{1}{s^{k}} \int_{M} h \gamma_{\varepsilon_{j}}\left(\frac{u}{s}\right) d\|V\|\right) & \geq \int_{G_{k}(M)} \frac{h}{s^{k}} \frac{\partial}{\partial s}\left(\gamma_{\varepsilon_{j}}\left(\frac{u}{s}\right)\right)\left|\nabla^{T^{\perp}} u\right|_{g}^{2} d V \\
& +\frac{1}{s^{k+1}}\left(\int_{G_{k}(M)} \gamma_{\varepsilon_{j}}\left(\frac{u}{s}\right)\langle\nabla h,(u \nabla u)\rangle_{g} d V\right) \\
& +\frac{(c(s)-1)}{s} \frac{k}{s^{k}} \int_{G_{k}(M)} h \gamma_{\varepsilon_{j}}\left(\frac{u}{s}\right) d\|V\| \\
& -\frac{\delta V\left(X_{s, \varepsilon}\right)}{s^{k+1}} .
\end{aligned}
$$

Letting $j \rightarrow \infty$, in virtue of Lebesgue dominated convergence theorem, we have for all $0<s<r_{0}$,

$$
\begin{aligned}
\lim _{j \rightarrow \infty} \int_{M} h(y) \gamma_{\varepsilon_{j}}\left(\frac{u(y)}{s}\right) d\|V\|(y) & =\int_{M} h(y) \chi_{B_{g}(\xi, s)}(y) d\|V\|(x) \\
& =\int_{B_{g}(\xi, s)} h(y) d\|V\|(y) .
\end{aligned}
$$

On the other hand, since $\left|\gamma_{\varepsilon_{j}}^{\prime}\left(\frac{u(x)}{s}\right)\right|<0$, for all $j \in \mathbb{N}$ and for all $x \in M$ such that $\varepsilon_{j} \leq u(x) / s \leq 1$, and $0<s<r_{0}$. Then

$$
\frac{\partial}{\partial s}\left(\gamma_{\varepsilon_{j}}\left(\frac{u(x)}{s}\right)\right)=\gamma_{\varepsilon_{j}}^{\prime}\left(\frac{u(x)}{s}\right)\left(\frac{-u(x)}{s^{2}}\right)>0 .
$$

Therefore, since $h \geq 0$

$$
\begin{aligned}
h(y) \frac{\varepsilon_{j}^{k}}{s^{k}} \frac{\partial}{\partial s}\left(\gamma_{\varepsilon_{j}}\left(\frac{u(y)}{s}\right)\right)\left|\nabla^{T^{\perp}} u(y)\right|_{g}^{2} & \leq h(y) \frac{\varepsilon_{j}^{k}}{u(y)^{k}} \frac{\partial}{\partial s}\left(\gamma_{\varepsilon_{j}}\left(\frac{u(y)}{s}\right)\right)\left|\nabla^{T^{\perp}} u(y)\right|_{g}^{2} \\
& \leq h(y) \frac{1}{s^{k}} \frac{\partial}{\partial s}\left(\gamma_{\varepsilon_{j}}\left(\frac{u(y)}{s}\right)\right)\left|\nabla^{T^{\perp}} u(y)\right|_{g}^{2}
\end{aligned}
$$

for all $n \in \mathbb{N}$. Integrating over $G_{k}(M)$,

$$
\begin{aligned}
\int_{G_{k}(M)} h \frac{\varepsilon_{j}^{k}}{s^{k}} \frac{\partial}{\partial s}\left(\gamma_{\varepsilon_{j}}\left(\frac{u}{s}\right)\right)\left|\nabla^{T^{\perp}} u\right|_{g}^{2} d V & \leq \int_{G_{k}(M)} h \frac{\varepsilon_{j}^{k}}{u^{k}} \frac{\partial}{\partial s}\left(\gamma_{\varepsilon_{j}}\left(\frac{u}{s}\right)\right)\left|\nabla^{T^{\perp}} u(x)\right|_{g}^{2} d V \\
& \leq \int_{G_{k}(M)} h \frac{1}{s^{k}} \frac{\partial}{\partial s}\left(\gamma_{\varepsilon_{j}}\left(\frac{u}{s}\right)\right)\left|\nabla^{T^{\perp}} u\right|_{g}^{2} d V
\end{aligned}
$$


and making $j \rightarrow \infty$, we have in distributional sense

$$
\lim _{j \rightarrow \infty} \int_{M} h(y) \frac{1}{s^{k}} \frac{\partial}{\partial s}\left(\gamma_{\varepsilon_{j}}\left(\frac{u(y)}{s}\right)\right)\left|\nabla^{S^{\perp}} u\right|_{g}^{2} d V(y, T)=\frac{d}{d s} J(s) .
$$

Finally, since $V$ satisfies $(A C)$, we have (see Remark 3.2.2)

$$
\delta V\left(X_{s, \varepsilon_{j}}\right)=-\int_{M} h(y) \gamma_{\varepsilon_{j}}\left(\frac{u(y)}{s}\right)\left\langle H_{g}(y),(u \nabla u)(y)\right\rangle_{g} d\|V\|(y),
$$

then, taking $X_{s}(y)=h(y) \chi_{B_{g}(\xi, s)}(y)(u \nabla u)(y)$,

$$
\left|\delta V\left(X_{s, \varepsilon_{j}}-X_{s}\right)\right| \leq \int_{M}|h(y)|\left|\gamma_{\varepsilon_{j}}\left(\frac{u(y)}{s}\right)-\chi_{]-\infty, 1[}\left(\frac{u(y)}{s}\right)\right|\left|H_{g}\right|_{g} d\|V\|(y) .
$$

By the Lebesgue Dominated Donvergence Theorem, we have $\gamma_{\varepsilon_{j}} \rightarrow \chi_{]-1,1[}$ in $L^{1}$ sense, then, letting $1 \leq p^{\prime}<+\infty$ be the conjugate exponent to $1<p \leq+\infty$, by Hölder inequality we have that

$$
\left\|\gamma_{\varepsilon_{j}}-\chi_{]-1,1}\right\|_{L^{p^{\prime}}\left(\|V\|, B_{g}(\xi, s)\right)} \rightarrow 0
$$

for all $s \in] 0, r_{0}\left[\right.$ and $1 \leq p^{\prime} \leq+\infty$. Therefore,

$$
\int_{M}\left\|H_{g}\right\||h|\left|\left(\gamma_{\varepsilon_{j}}-\chi_{]-1,1}\right)\left(\frac{u}{s}\right)\right| d\|V\| \leq\|h\|_{L^{\infty}(\|V\|)}\left\|H_{g}\right\|_{L^{p}}\left\|\gamma_{\varepsilon_{j}}-\chi_{]-1,1[}\right\|_{L^{p^{\prime}}} .
$$

Hence,

$$
\lim _{j \rightarrow \infty} \delta V\left(X_{s, \varepsilon_{j}}\right)=\frac{1}{s^{k+1}} \int_{B_{g}(\xi, s)}\langle H, h(y)(u \nabla u)(y)\rangle_{g} d\|V\|(y),
$$

and then the result follows from (4.6), (4.7), and (4.8)

Corollary 4.1.2.1 (Weighted Monotonicity Formula). Under the hypothesis of the theorem above, if $0<\sigma<\rho<r_{0}$. Then

$$
\begin{aligned}
\frac{1}{\rho^{k}} \int_{B_{g}(\xi, \rho)} h d\|V\|-\frac{1}{\sigma^{k}} \int_{B_{g}(\xi, \sigma)} h d\|V\| & \geq-\int_{\sigma}^{\rho} \frac{1}{s^{k}}\left(\int_{B_{g}(\xi, s)}\left|\nabla^{T} h\right| d V\right) \\
& -\int_{\sigma}^{\rho} \frac{1}{s^{k}}\left(\int_{B_{g}(\xi, s)} h\left(\left|H_{g}\right|_{g}-k c_{1}^{*}\right) d\|V\|\right) d s \\
& +(J(\rho)-J(\sigma)),
\end{aligned}
$$

where $J(s)$ is defined as in Theorem 4.1.2.

Proof. Integrating over $[\sigma, \rho] \subset\left[0, r_{0}\right]$, from Theorem 4.1.2 we have,

$$
\begin{aligned}
\int_{\sigma}^{\rho}\left(\frac{d}{d s}\left(\frac{1}{s^{k}} \int_{B_{g}(\xi, s)} h d\|V\|\right)\right) d s & \geq \int_{\sigma}^{\rho}\left(\frac{1}{s^{k+1}}\left(\int_{G_{k}\left(B_{g}(\xi, s)\right)}\left\langle\nabla^{T} h,(u \nabla u)\right\rangle_{g} d V\right)\right) d s \\
& +\int_{\sigma}^{\rho}\left(\frac{(c(s)-1)}{s} \frac{k}{s^{k}} \int_{B_{g}(\xi, s)} h d\|V\|\right) d s \\
& +\int_{\sigma}^{\rho}\left(\frac{1}{s^{k+1}} \int_{B_{g}(\xi, s)}\left\langle H_{g}, h(u \nabla u)\right\rangle_{g} d\|V\|\right) d s \\
& +\int_{\sigma}^{\rho} \frac{d}{d s} J(s) d s .
\end{aligned}
$$

Applying the Fundamental Theorem of Calculus to the left-hand term, we have 


$$
\int_{\sigma}^{\rho}\left(\frac{d}{d s}\left(\frac{1}{s^{k}} \int_{B_{g}(\xi, s)} h d\|V\|\right)\right)=\frac{1}{\rho^{k}} \int_{B_{g}(\xi, \rho)} h d\|V\|-\frac{1}{\sigma^{k}} \int_{B_{g}(\xi, \sigma)} h d\|V\| .
$$

To estimate the right-hand term, we precede in four steps. For the last term, again from the Fundamental Theorem of Calculus we have,

$$
\int_{\sigma}^{\rho} \frac{d}{d s} J(s) d s=J(\rho)-J(\rho)
$$

For the first term, we have

$$
\int_{\sigma}^{\rho}\left(\frac{1}{s^{k+1}}\left(\int_{G_{k}\left(B_{g}(\xi, s)\right)}\left\langle\nabla^{T} h,(u \nabla u)\right\rangle_{g} d V\right)\right) d s \geq-\int_{\sigma}^{\rho} \frac{1}{s^{k}}\left(\int_{G_{k}\left(B_{g}(\xi, s)\right)}\left|\nabla^{T} h\right|_{g} d V\right) d s
$$

Now, estimating the third and fourth term, (see Remark 4.1.2) we have

$$
\begin{aligned}
\int_{\sigma}^{\rho} \frac{1}{s^{k+1}} & \left(\int_{B_{g}(\xi, s)}\left\langle H_{g}, h(u \nabla u)\right\rangle_{g} d\|V\|\right) d s+\int_{\sigma}^{\rho} \frac{c(s)-1}{s^{k+1}}\left(\int_{B_{g}(\xi, s)} h d\|V\|\right) d s \\
& \geq-\int_{\sigma}^{\rho} \frac{1}{s^{k}}\left(\int_{B_{g}(\xi, s)}\left|H_{g}\right|_{g} h d\|V\|\right) d s+\int_{\sigma}^{\rho} \frac{k c_{1}^{*}}{s^{k}}\left(\int_{B_{g}(\xi, s)} h d\|V\|\right) d s \\
& \geq-\int_{\sigma}^{\rho} \frac{1}{s^{k}}\left(\int_{B_{g}(\xi, s)} h\left(\left|H_{g}(y)\right|_{g}-k c_{1}^{*}\right) d\|V\|\right) d s
\end{aligned}
$$

Hence, the result follows from (4.9), (4.10), (4.11), and (4.12)

Remark 4.1.3. Let $h: \operatorname{spt}\|V\| \subset M \rightarrow \mathbb{R}$ such that, $h \equiv 1$, and $J(s)$ as in Theorem 4.1.2 then, under the hypothesis of Theorem 4.1.2 as a particular case we have that there exists $c \in] 0,1[$ such that, fort all $0<s<r_{0}$

$$
\begin{aligned}
\frac{d}{d s}\left(\frac{\|V\|\left(B_{g}(\xi, s)\right)}{s^{k}}\right) & \geq \frac{d}{d s} J(s)+\frac{(c(s)-1)}{s} \frac{k}{s^{k}} \int_{B_{g}(\xi, s)} d\|V\| \\
& +\frac{1}{s^{k+1}} \int_{B_{g}(\xi, s)}\left\langle H_{g}, h(u \nabla u)\right\rangle_{g} d\|V\| .
\end{aligned}
$$

Now, assume that $V$ has locally bounded first variation in $B_{g}\left(\xi, 2 \rho_{0}\right)$ for some $\rho_{0}<r_{0}$, and let $\Lambda \geq 0$ such that

$$
\|\delta V\|\left(B_{g}(\xi, \rho)\right) \leq \Lambda\|V\|\left(B_{g}(\xi, \rho)\right), \quad \text { for } 0<\rho<\rho_{0},
$$

notice that the hypothesis above are much stronger than $(A C)$, and those implies that for all $X \in$ $\mathfrak{X}_{c}^{0}(M)$ with spt $X \subset B_{g}\left(\xi, s \rho_{0}\right)$,

$$
\delta V(X)=\int_{G_{k}(M)} \operatorname{div}_{S} X(y) d V(y, T)=-\int_{M}\left\langle H_{g}(y), X(y)\right\rangle_{g} d\|V\|(y) .
$$

Then is not so hard to see that (4.13) remains valid and $\left|H_{g}(y)\right|_{g} \leq \Lambda$ for $\|V\|$-a.e. $y \in B_{g}\left(\xi, \rho_{0}\right)$. Then, setting $c_{1}^{*}$ as in the Remark 4.1.2, 


$$
\begin{aligned}
\frac{d}{d s}\left(\frac{\|V\|\left(B_{g}(\xi, s)\right)}{s^{k}}\right) & \geq \frac{d}{d s} J(s)+\frac{k c_{1}^{*}}{s^{k}} \int_{B_{g}(\xi, s)} d\|V\|-\frac{1}{s^{k}} \int_{B_{g}(\xi, s)} \frac{|u \nabla u|_{g}}{s}\left|H_{g}\right|_{g} d\|V\| \\
& \geq \frac{d}{d s} J(s)-\frac{1}{s^{k}} \int_{B_{g}(\xi, s)}\left(\left|H_{g}(y)\right|_{g}-k c_{1}^{*}\right) d\|V\|(y) \\
& \geq \frac{d}{d s} J(s)-\left(\Lambda-k c_{1}^{*}\right) \frac{\|V\|\left(B_{g}(\xi, s)\right)}{s^{k}} .
\end{aligned}
$$

Hence,

$$
\frac{d}{d s}\left(\frac{\|V\|\left(B_{g}(\xi, s)\right)}{s^{k}}\right)+\left(\Lambda-k c_{1}^{*}\right) \frac{\|V\|\left(B_{g}(\xi, s)\right)}{s^{k}} \geq \frac{d}{d s} J(s)>0 .
$$

Finally, multiplying by $e^{\left(\Lambda-k c_{1}^{*}\right) s}$ we have

$$
e^{\left(\Lambda-k c_{1}^{*}\right) s} \frac{d}{d s}\left(\frac{\|V\|\left(B_{g}(\xi, s)\right)}{s^{k}}\right)+\left(\Lambda-k c_{1}^{*}\right) e^{\left(\Lambda-k c_{1}^{*}\right) s} \frac{\|V\|\left(B_{g}(\xi, s)\right)}{s^{k}} \geq 0,
$$

then

$$
\frac{d}{d s}\left(e^{\left(\Lambda-k c_{1}^{*}\right) s} \frac{\|V\|\left(B_{g}(\xi, s)\right)}{s^{k}}\right) \geq 0 .
$$

Therfore, the function

$$
\begin{aligned}
f:] 0, \rho_{0}[ & \rightarrow[0,+\infty[ \\
s & \mapsto e^{\left(\Lambda-k c_{1}^{*}\right) s} \frac{\|V\|\left(B_{g}(\xi, s)\right)}{s^{k}}
\end{aligned}
$$

in non-decreasing.

In the sequel we exploit the $L^{p}$ condition over the generalized mean curvature $H$ to get similar results, concerning to the monotonicity of this ratio.

\section{2 $\quad L^{p}$ Monotonicity Formula}

In this section we prove the monotonicity behaviour of the density ratio, as in Remark 4.1.3, but instead of the hypothesis of such we assume $L^{p}$ boundedness for $H$, i.e. under the hypothesis $(A C)$.

First to all, from now on we assume $(M, g)$ to be an $n$-dimensional complete Riemannian manifold satisfying $(B G)$, and let

$$
c^{*}=c^{*}\left(b, r_{0}\right)=\frac{c\left(b, r_{0}\right)-1}{r_{0}},
$$

where $c(b, s)$ is as in Remark ??.

Notice that, if $V \in \mathbf{V}_{k}\left(M^{n}\right)$ satisfy $(A C)$, and considering the special case on the Remark 4.1.3 we have in distributional sense that,

$$
\frac{d}{d s}\left(\frac{\|V\|\left(B_{g}(\xi, s)\right)}{s^{k}}\right) \geq \frac{d}{d s} J(s)+\frac{1}{s^{k+1}} \int_{B_{g}(\xi, s)}\left(k c^{*} s+\left\langle H_{g}, u \nabla u\right\rangle_{g}\right) d\|V\|,
$$

where $J(s)$ is as in Theorem 4.1.2. The equation above is the Intrinsic Riemannian version of the fundamental monotonicity identity of [Sim83], and from now on we refer to this as fundamental monotonicity inequality, also notice that, since

$$
J(s):=\int_{G_{k}\left(B_{g}(\xi, s)\right)} \frac{\left|\nabla^{T^{\perp}} u\right|_{g}^{2}}{r_{\xi}^{k}} d V \text { and }\|V\|\left(B_{g}(\xi, s)\right),
$$


are increasing in $s$, the inequality (4.15) holds also in the classical sense. Furthermore, from the analysis of this inequality, naturally follows a monotone behaviour of the density ratio.

Theorem 4.2.1. Given $\xi \in M$, let $0<\alpha \leq 1$ and $\Lambda$ be a positive constant. Let $V \in \mathbf{V}_{k}(M)$ satisfying $(A C)$ and for all $0<s<r_{0}$

$$
\frac{1}{\alpha} \int_{B_{g}(\xi, s)}\left|H_{g}(x)\right|_{g} d\|V\|(x) \leq \Lambda\left(\frac{s}{r_{0}}\right)^{\alpha-1}\|V\|\left(B_{g}(\xi, s)\right) .
$$

Then the function

$$
f(s):=e^{\lambda(s)} \frac{\|V\|\left(B_{g}(\xi, s)\right)}{s^{k}}
$$

is a non-decreasing function, where

$$
\lambda(s):=\left(\Lambda\left(\frac{s}{r_{0}}\right)^{\alpha-1}-c^{*} k\right) s,
$$

and in fact, for $0<\sigma<\rho \leq r_{0}$,

$$
e^{\lambda(\rho)} \frac{\|V\|\left(B_{g}(\xi, \rho)\right)}{\rho^{k}}-e^{\lambda(\sigma)} \frac{\|V\|\left(B_{g}(\xi, \sigma)\right)}{\sigma^{k}} \geq J(\rho)-J(\sigma) .
$$

Proof. Notice that it is enough to prove (4.16) to guarantee that $f$ is non-decreasing.

To prove (4.16), we multiply the fundamental monotonicity inequality (4.15) by $e^{\lambda(s)} \geq 1$, we have

$$
\begin{aligned}
e^{\lambda(s)} \frac{d}{d s}\left(\frac{\|V\|\left(B_{g}(\xi, s)\right)}{s^{k}}\right) & \geq e^{\lambda(s)} \frac{d}{d s} J(s)+\frac{e^{\lambda(s)}}{s^{k+1}} \int_{B_{g}(\xi, s)}\left(k c^{*} s+\left\langle H_{g}, u \nabla u\right\rangle_{g}\right) d\|V\| \\
& \geq \frac{d}{d s} J(s)-\frac{e^{\lambda(s)}}{s^{k+1}} \int_{B_{g}(\xi, s)}\left(\left|H_{g}\right|_{g}|u \nabla u|_{g}-k c^{*} s\right) d\|V\| \\
& \geq \frac{d}{d s} J(s)-\frac{e^{\lambda(s)}}{s^{k}} \int_{B_{g}(\xi, s)}\left(\left|H_{g}\right|_{g} \frac{|u \nabla u|_{g}}{s}-k c^{*}\right) d\|V\| \\
& \geq \frac{d}{d s} J(s)-\frac{e^{\lambda(s)}}{s^{k}} \int_{B_{g}(\xi, s)}\left(\left|H_{g}\right|_{g}-k c^{*}\right) d\|V\| .
\end{aligned}
$$

On the other hand, by hypothesis

$$
-\int_{B_{g}(\xi, s)}\left(|H|_{g}-k c^{*}\right) d\|V\| \geq-\left(\alpha \Lambda\left(\frac{s}{r_{0}}\right)^{\alpha-1}-k c^{*}\right)\|V\|\left(B_{g}(\xi, s)\right),
$$

therefore

$$
e^{\lambda(s)} \frac{d}{d s}\left(\frac{\|V\|\left(B_{g}(\xi, s)\right)}{s^{k}}\right)+\left(\alpha \Lambda\left(\frac{s}{r_{0}}\right)^{\alpha-1}-k c^{*}\right) e^{\lambda(s)} \frac{\|V\|\left(B_{g}(\xi, s)\right)}{s^{k}} \geq \frac{d}{d s} J(s) .
$$

Finally, since

$$
\frac{d}{d s} \lambda(s)=\alpha \Lambda\left(\frac{s}{r_{0}}\right)^{\alpha-1}-k c^{*}
$$

hence

$$
\frac{d}{d s}\left(e^{\lambda(s)} \frac{\|V\|\left(B_{g}(\xi, s)\right)}{s^{k}}\right) \geq J(s) \geq 0
$$

and (4.16) follows readily by integrating over the interval $[\sigma, \rho]$. 
Theorem 4.2.2. Given $\xi \in M$, let $p>k \geq 1$ and $\Gamma$ be a possitive constant. Let $V \in \mathbf{V}_{k}(M)$ satisfying $(A C)$ such that

$$
\left(\int_{B_{g}\left(\xi, r_{0}\right)}\left|H_{g}\right|_{g}^{p} d\|V\|\right)^{1 / p} \leq \Gamma
$$

Then there exists a positive constant $c_{2}^{*}=c_{2}^{*}\left(p, k, b, r_{0}\right)$ such that

$$
\left(\frac{\left(\|V\|\left(B_{g}(\xi, \sigma)\right)\right)}{\sigma^{k}}\right)^{\frac{1}{p}}-\left(\frac{\left(\|V\|\left(B_{g}(\xi, \rho)\right)\right)}{\rho^{k}}\right)^{\frac{1}{p}} \leq \frac{\Gamma+c_{2}^{*}}{p-k}\left(\rho^{1-k / p}-\sigma^{1-k / p}\right),
$$

for every $0<\sigma<\rho \leq r_{0}$.

Proof. From the Hölder inequality in the fundamental monotonicity inequality, Theorem (4.15) we have for all $0<s<r_{0}$ that

$$
\begin{aligned}
\frac{d}{d s}\left(\frac{\|V\|\left(B_{g}(\xi, s)\right)}{s^{k}}\right) & \geq \frac{d}{d s} J(s)+\frac{1}{s^{k+1}} \int_{B_{g}(\xi, s)}\left(k c^{*} s+\left\langle H_{g}, u \nabla u\right\rangle_{g}\right) d\|V\| \\
& \geq \frac{d}{d s} J(s)-\frac{1}{s^{k}} \int_{B_{g}(\xi, s)}\left(\left|H_{g}\right|_{g}-k c^{*}\right) d\|V\| \\
& \geq \frac{d}{d s} J(s)-\frac{1}{s^{k}} \int_{B_{g}(\xi, s)}\left|H_{g}\right|_{g} d\|V\|+k c^{*} \frac{\|V\|\left(B_{g}(\xi, s)\right)}{s^{k}} \\
& \geq \frac{d}{d s} J(s)-\frac{1}{s^{k}}\left(\|V\|\left(B_{g}(\xi, s)\right)\right)^{\frac{1}{p^{\prime}}}\left\|H_{g}\right\|_{L^{p}\left(B_{g}(\xi, s)\right)} \\
& +k c^{*} \frac{\|V\|\left(B_{g}(\xi, s)\right)}{s^{k}},
\end{aligned}
$$

where $p^{\prime}$ is the conjugate exponent to $p$. Now, by hypothesis we know that

$$
\left\|H_{g}\right\|_{L^{p}\left(\|V\|, B_{g}(\xi, s)\right)} \leq\left\|H_{g}\right\|_{L^{p}\left(\|V\|, B_{g}\left(\xi, r_{0}\right)\right)} \leq \Gamma,
$$

and, by (4.7) we also have that $\frac{d}{d s} J(s) \geq 0$ for all $0<s<r_{0}$, then

$$
\frac{d}{d s}\left(\frac{\|V\|\left(B_{g}(\xi, s)\right)}{s^{k}}\right) \geq-\frac{\left(\|V\|\left(B_{g}(\xi, s)\right)\right)^{\frac{1}{p^{\prime}}}}{s^{k}}\left(\Gamma-k c^{*}\left(\|V\|\left(B_{g}(\xi, s)\right) .\right)^{\frac{1}{p}}\right)
$$

Now, by Remarks 4.1.2 and ??, $c^{*} \leq 0$, then for all $0<s<r_{0}$

$$
\Gamma-k c^{*}\left(\|V\|\left(B_{g}(\xi, s)\right)\right)^{\frac{1}{p}} \leq \Gamma-k c^{*}\left(\|V\|\left(B_{g}\left(\xi, r_{0}\right)\right)\right)^{\frac{1}{p}} .
$$

Hence

$$
\frac{d}{d s}\left(\frac{\|V\|\left(B_{g}(\xi, s)\right)}{s^{k}}\right) \geq-\frac{\left(\|V\|\left(B_{g}(\xi, s)\right)\right)^{\frac{1}{p^{\prime}}}}{s^{k}}\left(\Gamma+c_{2}^{*}\right),
$$

where $c_{2}^{*}=c_{2}^{*}\left(k, p, b, r_{0}\right)$ is a positive constant such that

$$
-k c^{*}\left(\|V\|\left(B_{g}\left(\xi, r_{0}\right)\right)\right)^{\frac{1}{p}} \leq c_{2}^{*},
$$

such constant always exists because $\|V\|$ is a Radon measure.

On the other hand, since

$$
\frac{d}{d s}\left(\frac{\|V\|\left(B_{g}(\xi, s)\right)}{s^{k}}\right)^{\frac{1}{p}}=\frac{1}{p}\left(\frac{\|V\|\left(B_{g}(\xi, s)\right)}{s^{k}}\right)^{-\frac{1}{p^{\prime}}} \frac{d}{d s}\left(\frac{\|V\|\left(B_{g}(\xi, s)\right)}{s^{k}}\right),
$$


we have that

$$
\frac{d}{d s}\left(\frac{\|V\|\left(B_{g}(\xi, s)\right)}{s^{k}}\right)^{\frac{1}{p}} \geq \frac{1}{p}\left(\frac{\|V\|\left(B_{g}(\xi, s)\right)}{s^{k}}\right)^{-\frac{1}{p^{\prime}}}\left(-\frac{\left(\|V\|\left(B_{g}(\xi, s)\right)\right)^{\frac{1}{p^{\prime}}}}{s^{k}}\left(\Gamma+c_{2}^{*}\right)\right) \geq-\frac{\Gamma+c_{2}^{*}}{p s^{\frac{k}{p}}}
$$

and the result follows integrating the inequality above over $[\sigma, \rho] \subset] 0, r_{0}[$.

Corollary 4.2.2.1. If $H \in L_{l o c}^{p}\left(M, T M, \mathcal{H}_{g}^{k}\right)$ for some $p>k$, then the density

$$
\Theta^{k}(\|V\|, x):=\lim _{\rho \downarrow 0} \frac{\|V\|\left(B_{g}(x, \rho)\right)}{\omega_{k} \rho^{k}},
$$

does exists at every $x \in B_{g}\left(\xi, r_{0}\right)$. Furthermore, $\Theta^{k}(\|V\|, \cdot)$ is an upper-semi-continuous function in $M^{n}$, i.e.

$$
\Theta^{k}(\|V\|, x) \geq \limsup _{y \rightarrow x} \Theta^{k}(\|V\|, x) \quad \forall x \in B_{g}\left(\xi, r_{0}\right) .
$$

Proof. Let

$$
f(s):=\left(\frac{\|V\|\left(B_{g}(\xi, s)\right)}{s^{k}}\right)^{1 / p}+\frac{\Gamma+c_{1}^{*}}{p-k} s^{1-k / p} .
$$

By the Theorem 4.2.2, for $0<\sigma<\rho \leq r_{0}$

$$
\begin{aligned}
f(\rho)-f(\sigma)= & -\left(\left(\frac{\|V\|\left(B_{g}(\xi, \sigma)\right)}{\sigma^{k}}\right)^{1 / p}-\left(\frac{\|V\|\left(B_{g}(\xi, \rho)\right)}{\rho^{k}}\right)^{1 / p}\right) \\
& +\frac{\Gamma+c_{1}^{*}}{p-k}\left(\rho^{1-1 / p}-\sigma^{1-1 / p}\right) \geq 0 .
\end{aligned}
$$

Then $f$ is a non-decreasing function, therefore, the limit of $f(s)$ when $s \downarrow 0^{+}$, does exists and is nonnegative. Hence the limit

$$
\lim _{s \downarrow 0} \frac{\|V\|\left(B_{g}(\xi, s)\right)}{s^{k}}
$$

also exists, furthermore, is equal to

$$
\lim _{s \downarrow 0} \frac{\|V\|\left(\overline{B_{g}(\xi, s)}\right)}{s^{k}} .
$$

On the other hand, let $\varepsilon>0$ fixed, such that, if $0<\sigma<\rho, B_{g}(x, \rho+\varepsilon) \subset B_{g}\left(\xi, r_{0}\right)$ and $d_{g}(x, y)<\varepsilon$, then, we also deduce from Theorem 4.2.2 and the monotonicity of $\|V\|$ that

$$
\begin{aligned}
\left(\frac{\|V\|\left(B_{g}(y, \sigma)\right)}{\sigma^{k}}\right)^{1 / p} & \leq\left(\frac{\|V\|\left(B_{g}(y, \rho)\right)}{\rho^{k}}\right)^{1 / p}+\frac{\Gamma+c_{1}^{*}}{p-k} \rho^{1-k / p} \\
& \leq\left(\frac{\|V\|\left(B_{g}(x, \rho+\varepsilon)\right)}{\rho^{k}}\right)^{1 / p}+\frac{\Gamma+c_{1}^{*}}{p-k} \rho^{1-k / p}
\end{aligned}
$$

Letting $\sigma \downarrow 0$, we thus have

$$
\left(\Theta^{k}(\|V\|, y)\right)^{1 / p} \leq\left(\frac{\|V\|\left(B_{g}(x, \rho+\varepsilon)\right)}{\omega_{k}(\rho+\varepsilon)^{k / p}}\right)^{1 / p}+\frac{\Gamma+c_{1}^{*}}{\omega_{k}^{1 / p}(p-k)} \rho^{1-k / p} .
$$

Now, let $\delta>0$ be given and choose $\varepsilon \ll \rho<\delta$ so that

$$
\left(\frac{\|V\|\left(B_{g}(x, \rho+\varepsilon)\right)}{\omega_{k}(\rho+\varepsilon)^{k / p}}\right)<\left(\Theta^{k}(\|V\|, x)\right)^{1 / p}+\delta
$$


The inequality above gives

$$
\left(\Theta^{k}(\|V\|, y)\right)^{1 / p} \leq\left(\Theta^{k}(\|V\|, x)\right)^{1 / p}+\frac{\Gamma+c_{1}^{*}}{\omega_{k}^{1 / p}(p-k)} \rho^{1-k / p},
$$

provided $d_{g}(y, x)<\varepsilon$ and the desired upper-semi-continuity follows straightforward.

Proposition 4.2.1. Let $V \in \mathbf{V}_{k}(M), \xi \in M$ fixed, and assume that

$$
\|V\|\left(B_{g}(\xi, \sigma)\right) \leq \beta \sigma^{n} \quad \text { for } 0<\sigma<r_{0},
$$

then

$$
\int_{B_{g}(\xi, \rho)} d_{g}(x, \xi)^{\alpha-k} d\|V\|(x) \leq \frac{k \beta \rho^{\alpha}}{\alpha}
$$

for any $\rho \in] 0, r_{0}[$ and $0<\alpha<k$.

The proof of this is based in the following technical lemma.

Lemma 4.2.3. Let $(X, \mu)$ a measure space, $\gamma>0, f \in L^{1}(\mu), f \geq 0$, then

$$
\int_{0}^{\infty} t^{\gamma-1} \mu\left(E_{t}\right) d t=\frac{1}{\gamma} \int_{E_{0}} f^{\gamma}(x) d \mu(x),
$$

where $E_{t}:=\{x: f(x)>t\}$. More generally

$$
\int_{t_{0}}^{\infty} t^{\gamma-1} \mu\left(E_{t}\right) d t=\frac{1}{\gamma} \int_{E_{t_{0}}}\left(f^{\gamma}(x)-t_{0}^{\gamma}\right) d \mu(x)
$$

for each $t_{0} \geq 0$.

This is a clasical Lemma from measure theory, and its proof can be found in [Mat95] pg. 15.

Proof of Proposition 4.2.1. Let in the previous Lemma, $f(x)=|x-\xi|^{-1}, \gamma=k-\alpha$ and $t_{0}=1 / \rho$, then

$$
\int_{E_{1 / \rho}}\left(|x-\xi|_{g}^{\alpha-k}-\rho^{\alpha-k}\right) d\|V\|(x)=(\alpha-k) \int_{1 / \rho}^{\infty} t^{(k-\alpha)-1}\|V\|\left(E_{t}\right) d\|V\|(x) .
$$

Notice that

$$
E_{t}:=\{x: f(x)>t\}=\left\{x: \frac{1}{|x-\xi|_{g}}>t\right\}=\left\{x:|x-\xi|_{g}<1 / t\right\}:=B_{g}(\xi, 1 / t) .
$$

On the other hand, by hypothesis, we have

$$
\int_{1 / \rho}^{\infty} t^{(k-\alpha)-1}\|V\|\left(B_{g}(\xi, 1 / t)\right) d \mu(x) \leq \int_{1 / \rho}^{\infty} \frac{t^{(k-\alpha)-1} \beta}{t^{k}} d\|V\|(x)=\frac{\beta \rho^{\alpha}}{\alpha},
$$

for any $0<1 / t<r_{0}$. Putting together the information above in (4.18), we have

$$
\begin{aligned}
\int_{B_{g}(\xi, \rho)}\left(|x-\xi|_{g}^{\alpha-k}-\rho^{\alpha-k}\right) d\|V\| & =\int_{B_{g}(\xi, \rho)}\left(|x-\xi|_{g}^{\alpha-k}\right) d\|V\|-\int_{B_{g}(\xi, \rho)} \rho^{\alpha-k} d\|V\| \\
& =\int_{B_{g}(\xi, \rho)}\left(|x-\xi|_{g}^{\alpha-k}\right) d\|V\|-\rho^{\alpha-k}\|V\|\left(B_{g}(\xi, \rho)\right) \\
& \leq \frac{(k-\alpha) \beta \rho^{\alpha}}{\alpha}
\end{aligned}
$$


then,

$$
\begin{aligned}
\int_{B_{g}(\xi, \rho)}\left(|x-\xi|_{g}^{\alpha-k}\right) d\|V\| & \leq \frac{(k-\alpha) \beta \rho^{\alpha}}{\alpha}+\rho^{\alpha-k}\|V\|\left(B_{g}(\xi, \rho)\right) \\
& \leq \frac{(k-\alpha) \beta \rho^{\alpha}}{\alpha}+\beta \rho^{k} \\
& =\frac{k \beta \rho^{\alpha}}{\alpha},
\end{aligned}
$$

provided $\rho \in] 0, r_{0}[$. 


\section{Chapter 5}

\section{Poincaré and Sobolev Type Inequalities for Intrinsic Var- ifolds}

In this final section, a Poincaré and Sobolev-type inequalities are proved for non-negative functions defined on $S \subset M^{n}, \mathcal{H}_{g}^{k}$-rectifiable sets as a consequence of the Fundamental Weighted Monotonicity Inequality, Theorem 4.1.2, in the particular case in which $V:=\mathbf{v}(S, \theta)$, with $\theta \in L_{l o c}^{1}\left(\mathcal{H}_{g}^{k}\llcorner S)\right.$ and mean curvature vector $H_{g} \in L_{l o c}^{1}(\|V\|)$.

Therefore along this section we are under the assumptions of Theorem 4.1.2, i.e.

$(B G)$ : We say that a complete Riemannian manifold $\left(M^{n}, g\right)$ satisfy $(B G)$, if $S e c_{g}<b$ for some constant $b \in \mathbb{R}$ and for every $\xi \in M$ there is $r_{0}$ such that $0<r_{0}<i n j_{\xi}(M, g)$ and in case $b>0$ we assume furthermore $b r_{0}<\pi$.

$(A C)$ : We say that $V \in \mathbf{V}_{k}(M)$ satisfies the $(A C)$ condition, whenever

$$
\delta V(X) \leq C(V)\left(\int_{B_{g}\left(\xi, r_{0}\right)}|X|^{\frac{p}{p-1}} d\|V\|\right)^{\frac{p-1}{p}}, \forall X \in \mathfrak{X}_{c}^{1}\left(B_{g}\left(\xi, r_{0}\right)\right),
$$

for some $p>k$.

Furthermore, we need to explain some notation: in what follows $\xi \in M$ is a fixed point and $r_{0}<$ $\operatorname{inj}_{\xi}(M, g)$ such that $r_{0} \cot _{b}\left(r_{0}\right)>0$, according to Definition 4.1.1.

\subsection{Poincaré type inequality}

The Poincaré type inequality which we proof is a consequence of the following lemma, which is a direct application of the formula (4.1.2).

Lemma 5.1.1. Assume $\left(M^{n}, g\right)$ satisfying $(B G)$ and $V \in \mathbf{V}_{k}\left(M^{n}\right)$ satisfying $(A C)$. Let $\Lambda>0$ such that $\left|H_{g}\right|_{g} \leq \Lambda$, and $h \in C^{1}(M)$, then for $\|V\|$-a.e. $\xi \in \operatorname{spt}\|V\|$, and for all $0<\rho<r_{0}$

$$
h(\xi) \leq e^{\left(\Lambda+c^{*} k\right) \rho}\left(\frac{1}{\omega_{k}} \rho^{k} \int_{B_{g}(\xi, \rho)} h d\|V\|+\int_{B_{g}(\xi, \rho)} \frac{\left|\nabla_{g}^{S} h\right|_{g}}{r_{\xi}^{k-1}} d\|V\|\right),
$$

where $c^{*}$ is as in Remark 4.1.2.

Proof. By Hypothesis $(B G)$ and $(A C)$ we are in the conditions of Theorem 4.1.2, then, for $\xi \in$ spt $\|V\|$ and for all $0<s<r_{0}$ we have 


$$
\begin{aligned}
\frac{d}{d s}\left(\frac{1}{s^{k}} \int_{B_{g}(\xi, s)} h \gamma_{\varepsilon}\left(\frac{u}{s}\right) d\|V\|\right) & \geq \int_{B_{g}(\xi, s)} \frac{h}{s^{k}} \frac{\partial}{\partial s}\left(\gamma_{\varepsilon}\left(\frac{u}{s}\right)\right)\left|\nabla_{g}^{\perp} u\right|_{g}^{2} d\|V\| \\
& +\frac{1}{s^{k}} \int_{B_{g}(\xi, s)} \gamma_{\varepsilon}\left(\frac{u}{s}\right)\left\langle\nabla_{g}^{S} h, u \nabla_{g} u\right\rangle_{g} d\|V\| \\
& +\frac{k c^{*}}{s^{k}} \int_{B_{g}(\xi, s)} h \gamma_{\varepsilon}\left(\frac{u}{s}\right) d\|V\| \\
& +\frac{1}{s^{k+1}} \int_{B_{g}(\xi, s)} \gamma_{\varepsilon}\left(\frac{u}{s}\right)\left\langle h H_{g}, u \nabla_{g} u\right\rangle_{g} d\|V\|,
\end{aligned}
$$

where $\gamma_{\varepsilon} \in C^{1}(\mathbb{R})$ is, such that, given $0<\varepsilon<1$,

$$
\gamma_{\varepsilon}(s):= \begin{cases}1, & \text { if } \quad s<\varepsilon \\ 0, & \text { if } \quad s>1\end{cases}
$$

and

$$
\gamma_{\varepsilon}^{\prime}(s)<0 \quad \text { if } \quad \varepsilon<s<1
$$

Then

$$
\begin{aligned}
\frac{d}{d s}\left(\frac{1}{s^{k}} \int_{B_{g}(\xi, s)} h \gamma_{\varepsilon}\left(\frac{u}{s}\right) d\|V\|\right) & \geq \frac{1}{s^{k+1}} \int_{B_{g}(\xi, s)} \gamma_{\varepsilon}\left(\frac{u}{s}\right)\left\langle H_{g} h+\nabla_{g}^{S} h, u \nabla_{g} u\right\rangle_{g} d\|V\| \\
& +\frac{c^{*} k}{s^{k}} \int_{B_{g}(\xi, s)} h \gamma_{\varepsilon}\left(\frac{u}{s}\right) d\|V\| \\
& \geq-\frac{1}{s^{k+1}} \int_{B_{g}(\xi, s)} \gamma_{\varepsilon}\left(\frac{u}{s}\right)\left|h H_{g}+\nabla_{g}^{S} h\right|_{g} u d\|V\| \\
& +\frac{c^{*} k}{s^{k}} \int_{B_{g}(\xi, s)} h \gamma_{\varepsilon}\left(\frac{u}{s}\right) d\|V\|,
\end{aligned}
$$

therefore

$$
\begin{aligned}
\frac{d}{d s}\left(\frac{1}{s^{k}} \int_{B_{g}(\xi, s)} \gamma_{\varepsilon}\left(\frac{u}{s}\right) h d\|V\|\right) & \geq-\frac{1}{s^{k+1}} \int_{B_{g}(\xi, s)} \gamma_{\varepsilon}\left(\frac{u}{s}\right) u\left|\nabla_{g}^{S} h\right|_{g} d\|V\| \\
& -\frac{1}{s^{k}} \int_{B_{g}(\xi, s)} \gamma_{\varepsilon}\left(\frac{u}{s}\right) h\left(\left|H_{g}\right|_{g}-c^{*} k\right) d\|V\| .
\end{aligned}
$$

Since, by hypothesis, $\left|H_{g}\right|_{g} \leq \Lambda$, we get,

$$
\begin{aligned}
\frac{d}{d s}\left(\frac{1}{s^{k}} \int_{B_{g}(\xi, s)} \gamma_{\varepsilon}\left(\frac{u}{s}\right) h d\|V\|\right) & \geq-\frac{1}{s^{k+1}} \int_{B_{g}(\xi, s)} \gamma_{\varepsilon}\left(\frac{u}{s}\right) u\left|\nabla_{g}^{S} h\right|_{g} d\|V\| \\
& -\frac{\Lambda+c^{*} k}{s^{k}} \int_{B_{g}(\xi, s)} \gamma_{\varepsilon}\left(\frac{u}{s}\right) h d\|V\| .
\end{aligned}
$$

Now, let $\left\{\varepsilon_{j}\right\}_{j \in \mathbb{N}}$ a sequence of real numbers such that $\varepsilon \uparrow 1$ as $j \rightarrow \infty$, and $\left\{\gamma_{\varepsilon_{j}}\right\}_{j \in \mathbb{N}} \subset C^{1}(\mathbb{R})$ such that

$$
\gamma_{\varepsilon_{j}}(s):=\left\{\begin{array}{lll}
1, & \text { if } & s<\varepsilon_{j} \\
0, & \text { if } & s>1,
\end{array}\right.
$$


and

$$
\gamma_{\varepsilon_{j}}^{\prime}(s)<0, \quad \text { if } \quad \varepsilon_{j}<s<1
$$

and $\varepsilon_{j} \rightarrow \chi_{-\infty, 1[}$ pointwise from below. Thus, reasoning as above we have (as in the proof of Theorem 4.1.2) that for all $j \in \mathbb{N}$, and for all $0<s<r_{0}$

$$
\begin{aligned}
\frac{d}{d s}\left(\frac{1}{s^{k}} \int_{B_{g}(\xi, s)} \gamma_{\varepsilon_{j}}\left(\frac{u}{s}\right) h d\|V\|\right) & \geq-\frac{1}{s^{k+1}} \int_{B_{g}(\xi, s)} \gamma_{\varepsilon_{j}}\left(\frac{u}{s}\right) u\left|\nabla_{g}^{S} h\right|_{g} d\|V\| \\
& -\frac{\Lambda+c^{*} k}{s^{k}} \int_{B_{g}(\xi, s)} \gamma_{\varepsilon_{j}}\left(\frac{u}{s}\right) h d\|V\| .
\end{aligned}
$$

Multiply the above inequality by $e^{\tilde{\lambda}(s)}$ and letting $j \rightarrow \infty$ we have in distributional sense in $s$,

$$
\begin{aligned}
e^{\tilde{\lambda}(s)} \frac{d}{d s}\left(\frac{1}{s^{k}} \int_{B_{g}(\xi, s)} h d\|V\|\right) & +e^{\tilde{\lambda}(s)} \frac{\Lambda+c^{*} k}{s^{k}} \int_{B_{g}(\xi, s)} h d\|V\| \\
& \geq-e^{\tilde{\lambda}(s)} \frac{1}{s^{k+1}} \int_{B_{g}(\xi, s)} u\left|\nabla_{g}^{S} h\right|_{g} d\|V\|,
\end{aligned}
$$

where

$$
\tilde{\lambda}(s):=\left(\Lambda+c^{*} k\right) s,
$$

therefore, since $\tilde{\lambda}^{\prime}(s)=\Lambda+c^{*} k$, we have

$$
\frac{d}{d s}\left(\frac{e^{\tilde{\lambda}(s)}}{s^{k}} \int_{B_{g}(\xi, s)} h d\|V\|\right) \geq-\frac{e^{\tilde{\lambda}(s)}}{s^{k+1}} \int_{b_{g}(\xi, s)} u\left|\nabla_{g}^{S} h\right|_{g} d\|V\|,
$$

hence, integrating over $[\sigma, \rho] \subset] 0, r_{0}[$,

$$
\begin{aligned}
\frac{e^{\tilde{\lambda}(\rho)}}{\rho^{k}} \int_{B_{g}(\xi, \rho)} h d\|V\| & -\frac{e^{\tilde{\lambda}(\sigma)}}{\sigma^{k}} \int_{B_{g}(\xi, \sigma)} h d\|V\|=\int_{\sigma}^{\rho} \frac{d}{d s}\left(\frac{e^{\tilde{\lambda}(s)}}{s^{k}} \int_{B_{g}(\xi, s)} h d\|V\|\right) \\
& \geq-\int_{\sigma}^{\rho} \frac{e^{\tilde{\lambda}(s)}}{s^{k+1}} \int_{B_{g}(\xi, s)} u\left|\nabla_{g}^{S} h\right|_{g} d\|V\| \\
& \geq-e^{\tilde{\lambda}(\rho)} \int_{\sigma}^{\rho} \frac{1}{s^{k+1}}\left(\int_{B_{g}(\xi, s)} u\left|\nabla_{g}^{S} h\right|_{g} d\|V\|\right) d s
\end{aligned}
$$

then, rearranging terms,

$$
\frac{1}{\omega_{k} \sigma_{k}} \int_{B_{g}(\xi, \sigma)} h d\|V\| \leq e^{\tilde{\lambda}(\rho)}\left(\frac{1}{\omega_{k} \rho_{k}} \int_{B_{g}(\xi, \rho)} h d\|V\|+\frac{1}{\omega_{k}} \int_{\sigma}^{\rho} \frac{1}{s^{k+1}}\left(\int_{B_{g}(\xi, s)} u\left|\nabla_{g}^{S} h\right|_{g} d\|V\|\right) d s\right) .
$$

Now, letting $\tau=1 / s$ in the inequality above, we have that

$$
\int_{\sigma}^{\rho}\left(\frac{1}{s}\right)^{k+1}\left(\int_{B_{g}(\xi, s)} u\left|\nabla_{g}^{S} h\right|_{g} d\|V\|\right) d s=-\int_{\frac{1}{\sigma}}^{\frac{1}{\rho}} \tau^{k-1} \int_{B_{g}\left(\xi, \frac{1}{\tau}\right)} u\left|\nabla_{g}^{S} h\right|_{g} d\|V\|,
$$

then, if we let for $A \subset M$

$$
\nu(A):=\int_{A} u\left|\nabla_{g}^{S} h\right|_{g} d\|V\|,
$$


since $u$ is an increasing function, $\nu$ defines a measure on $(M, g)$, so by Lemma 4.2 .3

$$
\begin{aligned}
\int_{\sigma}^{\rho} \frac{1}{s^{k+1}}\left(\int_{B_{g}(\xi, s)} u\left|\nabla_{g}^{S} h\right|_{g} d\|V\|\right) d s & =-\int_{\frac{1}{\sigma}}^{\frac{1}{\rho}} \tau^{k-1} \nu\left(B_{g}\left(\xi, \frac{1}{\tau}\right)\right) d \tau \\
& =\int_{\frac{1}{\rho}}^{\frac{1}{\sigma}} \tau^{k-1} \nu\left(\left\{x \in M: \frac{1}{u(x)}>\tau\right\}\right) d \tau \\
& =\frac{1}{k} \int_{\left\{\frac{1}{\rho}<\frac{1}{u}<\frac{1}{\sigma}\right\}} \frac{1}{u(x)^{k}}-\left(\frac{1}{\sigma^{k}}-\frac{1}{\rho^{k}}\right) d \nu \\
& =\int_{B_{g}(\xi, \rho) \backslash B_{g}(\xi, \sigma)} \frac{1}{u^{k}}-\left(\frac{1}{\sigma^{k}}-\frac{1}{\rho^{k}}\right) d \nu \\
& \leq \frac{1}{k} \int_{B_{g}(\xi, \rho)} \frac{1}{u^{k}} d \nu \\
& =\frac{1}{k} \int_{B_{g}(\xi, \rho)} \frac{u\left|\nabla_{g}^{S} h\right|_{g}}{u^{k}} d\|V\| \\
& =\frac{1}{k} \int_{B_{g}(\xi, \rho)} \frac{\left|\nabla_{g}^{S} h\right|_{g}}{u^{k-1}} d\|V\| .
\end{aligned}
$$

Thus

$$
\frac{1}{\omega_{k} \sigma^{k}} \int_{B_{g}(\xi, \sigma)} h d\|V\| \leq e^{\tilde{\lambda}(\rho)}\left(\frac{1}{\omega_{k} \rho^{k}} \int_{B_{g}(\xi, \rho)} h d\|V\|+\frac{1}{k \omega_{k}} \int_{B_{g}(\xi, \rho)} \frac{\left|\nabla_{g}^{S} h\right|_{g}}{r_{\xi}^{k-1}} d\|V\|\right) .
$$

Finally, let $\sigma \downarrow 0$, and the result follows, since, $\xi \in \operatorname{spt}\|V\|$ implies $\Theta^{k}(\|V\|, \xi)=1$ for $\|V\|$-a.e. $\xi$, therefore $\xi$ is a Lebesgue point $\|V\|$-a.e.

Theorem 5.1.2 (Poincaré-Type Inequality for Intrinsic Varifolds). Let $\left(M^{n}, g\right)$ be a complete Riemannian manifold satisfying $(B G)$, let $V:=\mathbf{v}(S, \theta)$ a rectifiable varifold satisfying $(A C)$. Suppose: $h \in C^{1}(M), h \geq 0, B_{g}(\xi, 2 \rho) \subset B_{g}\left(\xi, r_{0}\right)$ for $\xi \in S$ fixed, $\left|H_{g}\right|_{g} \leq \Lambda$ for some $\Lambda>0, \theta>1\|V\|$-a.e. in $B_{g}\left(\xi, r_{0}\right)$ and for some $0<\alpha<1$

$$
\begin{aligned}
\|V\|\left(\left\{x \in B_{g}(\xi, \rho): h(x)>0\right\}\right) & \leq \omega_{k}(1-\alpha) \rho^{k} \quad \text { and }, \\
e^{\left(\Lambda+k c^{*}\right) \rho} & \leq 1+\alpha .
\end{aligned}
$$

Suppose also that, for some constant $\Gamma>0$

$$
\|V\|\left(B_{g}(\xi, 2 \rho)\right) \leq \Gamma \rho^{k} .
$$

Then there are constants $\left.\beta:=\beta\left(k, \alpha, r_{0}, b\right) \in\right] 0, \frac{1}{2}\left[\right.$ and $C:=C\left(k, \alpha, r_{0}, b\right)>0$ such that

$$
\int_{B_{g}(\xi, 2 \rho)} h d\|V\| \leq C \rho \int_{B_{g}(\xi, \rho)}\left|\nabla_{g}^{S} h\right|_{g} d\|V\| .
$$

Proof. Take $\beta \in] 0,1 / 2[$ an arbitrary parameter, to be specified later and $\tilde{\lambda}(s)$ as in (5.3). Applying the previous Lemma with

$$
\eta \in B_{g}(\xi, \beta \rho) \cap \operatorname{spt}\|V\|,
$$

in place of $\xi$, thence, for all $0<\rho<r_{0}$

$$
h(\eta) \leq e^{\tilde{\lambda}((1-\beta) \rho)}\left(\frac{1}{\omega_{k}(1-\beta)^{k} \rho^{k}} \int_{B_{g}(\eta,(1-\beta) \rho)} h d\|V\|+\frac{1}{k \omega_{k}} \int_{B_{g}(\eta,(1-\beta) \rho)} \frac{\left|\nabla_{g}^{S} h\right|_{g}}{\left.r_{\eta}^{k-1} d\|V\|\right)} .\right.
$$


Since $\rho / 2<(1-\beta) \rho<\rho$, we have $B_{g}(\eta,(1-\beta) \rho) \subset B_{g}(\xi, \rho)$ and

$$
h(\eta) \leq e^{\tilde{\lambda}(\rho)}\left(\frac{1}{\omega_{k}(1-\beta)^{k} \rho^{k}} \int_{B_{g}(\xi, \rho)} h d\|V\|+\frac{1}{k \omega^{k}} \int_{B_{g}(\xi, \rho)} \frac{\left|\nabla_{g}^{S} h\right|_{g}}{r_{\eta}^{k-1}} d\|V\|\right) .
$$

Now, let $t_{0} \geq 0$ fixed, and $\gamma \in C^{1}(\mathbb{R})$ a fixed non-decreasing function with

$$
\gamma(t)=0 \text {, if } t \leq 0 \text { and } \gamma(t) \leq 1 \text { if } t>0,
$$

and apply (5.7) with $f(x):=\gamma\left(h(x)-t_{0}\right)$ in place of $h$, then

$$
f(\eta) \leq e^{\tilde{\lambda}(\rho)}\left(\frac{1}{\omega_{k}(1-\beta)^{k} \rho^{k}} \int_{B_{g}(\xi, \rho)} f d\|V\|+\frac{1}{k \omega^{k}} \int_{B_{g}(\xi, \rho)} \frac{\gamma^{\prime}\left(h-t_{0}\right)\left|\nabla_{g}^{S} h\right|_{g}}{r_{\eta}^{k-1}} d\|V\|\right) .
$$

By hypothesis (5.5) we know that:

$$
e^{\tilde{\lambda}(\rho)} \leq 1+\alpha
$$

and that

$$
\|V\|\left(\left\{x \in B_{g}(\xi, \rho): h(x)>0\right\}\right) \leq \omega_{k}(1-\alpha) \rho^{k},
$$

hence,

$$
\begin{aligned}
f(\eta) & \leq(1+\alpha)\left(\frac{1}{\omega_{k}(1-\beta)^{k} \rho^{k}} \int_{B_{g}(\xi, \rho)} f d\|V\|+\frac{1}{k \omega^{k}} \int_{B_{g}(\xi, \rho)} \frac{\gamma^{\prime}\left(h-t_{0}\right)\left|\nabla_{g}^{S} h\right|_{g}}{r_{\eta}^{k-1}} d\|V\|\right) \\
& \leq(1+\alpha)\left(\frac{\|V\|\left(\{h>0\} \cap B_{g}(\xi, \rho)\right)}{\omega_{k}(1-\beta)^{k} \rho^{k}}+\frac{1}{k \omega^{k}} \int_{B_{g}(\xi, \rho)} \frac{\gamma^{\prime}\left(h-t_{0}\right)\left|\nabla_{g}^{S} h\right|_{g}}{r_{\eta}^{k-1}} d\|V\|\right) \\
& \leq \frac{1-\alpha^{2}}{(1-\beta)^{k}}+\frac{1+\alpha}{k \omega_{k}} \int_{B_{g}(\xi, \rho)} \frac{\gamma^{\prime}\left(h-t_{0}\right)\left|\nabla_{g}^{S} h\right|_{g}}{r_{\eta}^{k-1}} d\|V\| .
\end{aligned}
$$

Now, take $\beta:=\beta(k, \alpha) \in] 0,1 / 2[$ small enough, such that

$$
\frac{1-\alpha^{2}}{(1-\beta)^{k}} \leq 1-\frac{\alpha^{2}}{2}
$$

then

$$
\frac{\alpha^{2}}{2}+(f(\eta)-1) \leq \frac{(1+\alpha)}{k \omega_{k}} \int_{B_{g}(\xi, \rho)} \frac{\gamma^{\prime}\left(h(x)-t_{0}\right)\left|\nabla_{g}^{S} h(x)\right|_{g}}{r_{\eta}^{k-1}} d\|V\|(x) .
$$

Therefore, for any $\eta \in B_{g}(\xi, \beta \rho) \cap \operatorname{spt}\|V\|$ such that $f(\eta) \geq 1$,

$$
1 \leq \frac{2(1+\alpha)}{\alpha^{2} k \omega_{k}} \int_{B_{g}(\xi, \rho)} \frac{\gamma^{\prime}\left(h(x)-t_{0}\right)\left|\nabla_{g}^{S} h(x)\right|_{g}}{r_{\eta}^{k-1}} d\|V\|(x) .
$$

Let $\varepsilon>0$ given, and choose $\gamma$ such that, $\gamma(t) \equiv 1$ for $t \geq \varepsilon$, then $f \equiv 1$ for $\eta \in B_{g}(\xi, \beta \rho) \cap E_{t_{0}+\varepsilon}$, where $E_{\tau}:=\{x \in \operatorname{spt}\|V\|: h(x)>\tau\}$, and so (5.8) remains valid. Then integrating in booth sides 
over $B_{g}(\xi, \beta \rho) \cap E_{t_{0}+\varepsilon}$, we get

$$
\begin{aligned}
\|V\|\left(B_{g}(\xi, \beta \rho) \cap E_{t_{0}+\varepsilon}\right) & \leq \frac{2(1+\alpha)}{\alpha^{2} k \omega_{k}} \int_{B_{g}(\xi, \beta \rho) \cap E_{t_{0}+\varepsilon}}\left(\int_{B_{g}(\xi, \rho)} \frac{\gamma^{\prime}\left(h(x)-t_{0}\right)\left|\nabla_{g}^{S} h(x)\right|_{g}}{r_{\eta}^{k-1}} d\|V\|(x)\right) d\|V\|(\eta) \\
& =\frac{2(1+\alpha)}{\alpha^{2} k \omega_{k}} \int_{B_{g}(\xi, \rho)} \gamma^{\prime}\left(h(x)-t_{0}\right)\left|\nabla_{g}^{S} h(x)\right|_{g}\left(\int_{B_{g}(\xi, \beta \rho) \cap E_{t_{0}+\varepsilon}} \frac{1}{r_{\eta}^{k-1}} d\|V\|(\eta)\right) d\|V\|(x) \\
& \leq \frac{2(1+\alpha)}{\alpha^{2} k \omega_{k}} \int_{B_{g}(\xi, \rho)} \gamma^{\prime}\left(h(x)-t_{0}\right)\left|\nabla_{g}^{S} h(x)\right|_{g}\left(\int_{B_{g}(\xi, \rho)} \frac{1}{r_{\eta}^{k-1}} d\|V\|(\eta)\right) d\|V\|(x) .
\end{aligned}
$$

Now, since by hypothesis $\|V\|\left(B_{g}(\xi, 2 \rho)\right) \leq \Gamma \rho^{k}$, we have, by Proposition 4.2 .1 for all $0<\rho<r_{0}$ (notice that $\rho$ has to satisfy $0<\rho<2 \rho<r_{0}$ )

$$
\int_{B_{g}(\xi, \rho)} \frac{1}{r_{\eta}^{k-1}} d\|V\|(\eta) \leq k \Gamma \rho
$$

then

$$
\|V\|\left(B_{g}(\xi, \beta \rho) \cap E_{t_{0}+\varepsilon}\right) \leq\left(\frac{2(1+\alpha) \Gamma}{\alpha^{2} \omega_{k}}\right) \rho \int_{B_{g}(\xi, \rho)} \gamma^{\prime}\left(h(x)-t_{0}\right)\left|\nabla_{g}^{S} h(x)\right|_{g} d\|V\|(x) .
$$

Integrating over $\left[0, \infty\left[\right.\right.$ in $t_{0}$,

$$
\begin{aligned}
\int_{0}^{\infty}\|V\|\left(B_{g}(\xi, \beta \rho) \cap E_{t_{0}+\varepsilon}\right) d t_{0} & \leq\left(\frac{2(1+\alpha) \Gamma}{\alpha^{2} \omega_{k}}\right) \rho \int_{0}^{\infty}\left(\int_{B_{g}(\xi, \rho)} \gamma^{\prime}\left(h(x)-t_{0}\right)\left|\nabla_{g}^{S} h(x)\right|_{g} d\|V\|(x)\right) d t_{0} \\
& =\left(\frac{2(1+\alpha) \Gamma}{\alpha^{2} \omega_{k}}\right) \rho \int_{0}^{\infty}\left(\int_{B_{g}(\xi, \rho)}-\frac{\partial}{\partial t_{0}}\left(\gamma\left(h(x)-t_{0}\right)\right)\left|\nabla_{g}^{S} h(x)\right|_{g} d\|V\|(x)\right) d t_{0} \\
& =\left(\frac{2(1+\alpha) \Gamma}{\alpha^{2} \omega_{k}}\right) \rho \int_{B_{g}(\xi, \rho)}\left|\nabla_{g}^{S} h(x)\right|_{g}\left(-\frac{d}{d s} \int_{0}^{\infty} \gamma\left(h(x)-t_{0}\right) d t_{0}\right) d\|V\|(x) \\
& \leq\left(\frac{2(1+\alpha) \Gamma}{\alpha^{2} \omega_{k}}\right) \rho \int_{B_{g}(\xi, \rho)}\left|\nabla_{g}^{S} h(x)\right|_{g} \gamma(h(x)) d\|V\|(x) \\
& \leq\left(\frac{2(1+\alpha) \Gamma}{\alpha^{2} \omega_{k}}\right) \rho \int_{B_{g}(\xi, \rho)}\left|\nabla_{g}^{S} h(x)\right|_{g} d\|V\|(x) .
\end{aligned}
$$

To calculate the left term, let $\tilde{A}_{\tau}:=\{x \in \operatorname{spt}\|V\|:(h-\varepsilon)(x)>\tau\}=A_{\tau+\varepsilon}$, then by Lemma 4.2.3

$$
\begin{aligned}
\int_{0}^{\infty}\|V\|\left(B_{g}(\xi, \beta \rho) \cap E_{t_{0}+\varepsilon}\right) & =\int_{0}^{\infty}\|V\|\left\llcorner\left(B_{g}(\xi, \beta \rho)\right)\left(\tilde{A}_{\tau}\right) d t_{0}\right. \\
& =\int_{\tilde{A}_{0}}(h-\varepsilon) d\|V\|\left\llcorner\left(B_{g}(\xi, \beta \rho)\right)\right. \\
& =\int_{B_{g}(\xi, \beta \rho) \cap A_{\varepsilon}}(h-\varepsilon) d\|V\| .
\end{aligned}
$$

Sumarizing,

$$
\int_{B_{g}(\xi, \beta \rho) \cap A_{\varepsilon}}(h(x)-\varepsilon) d\|V\|(x) \leq C \rho \int_{B_{g}(\xi, \rho)}\left|\nabla_{g}^{S} h(x)\right|_{g} d\|V\|(x),
$$

where

$$
C:=C\left(k, \alpha, \Gamma, r_{0}\right):=\frac{2(1+\alpha) \Gamma}{\alpha^{2} \omega_{k}} .
$$

Finally, letting $\varepsilon \downarrow 0$, the result follows. 


\subsection{Sobolev type Inequality}

Finally, in order to prove the desired Sobolev type inequality, we use the Theorem 4.1.2 to derive an inequality in which the tangential derivative of $h$ appears, this joint with a calculus lemma and the Lemma 4.2.3 gives a "local " version of the desired inequality, which via a covering argument (c.f. Theorem 2.1.3) implies a "global" Sobolev inequality, where the constant derived depends only on the dimension of the ambient manifold and does not depends on the geometry, nevertheless this constant is not optimal.

Lemma 5.2.1. Assume $\left(M^{n}, g\right)$ satisfying $(B G)$ and $V:=\mathbf{v}(S, \theta)$, with $S \subset M \mathcal{H}_{g}^{k}$-rectifiable, and $\theta \in L_{l o c}^{1}(\|V\|)$, such that $(A C)$ is satisfied. Let $h \in C^{1}(M)$ non-negative, then for all $\xi \in$ spt $\|V\|$ and for all $0<\rho<r_{0}$

$$
\frac{1}{\omega_{k} \sigma^{k}} \int_{B_{g}(\xi, \sigma)} h d\|V\| \leq \frac{1}{\omega_{k} \rho^{k}} \int_{B_{g}(\xi, \rho)} h d\|V\|+\int_{\sigma}^{\rho} \frac{1}{s^{k}}\left(\int_{B_{g}(\xi, s)}\left|\nabla_{g}^{S} h\right|_{g}+h\left(\left|H_{g}\right|_{g}-c^{*} k\right) d\|V\|\right) d s
$$

where $c^{*}$ is as in Remark 4.1.2.

Proof. By Hypothesis $(B G)$ and $(A C)$ we are in the conditions of Theorem 4.1.2, then, for $\xi \in$ spt $\|V\|$ and for all $0<s<r_{0}$

$$
\begin{aligned}
\frac{d}{d s}\left(\frac{1}{s^{k}} \int_{B_{g}(\xi, s)} h \gamma_{\varepsilon}\left(\frac{u}{s}\right) d\|V\|\right) & \geq \int_{B_{g}(\xi, s)} \frac{h}{s^{k}} \frac{\partial}{\partial s}\left(\gamma_{\varepsilon}\left(\frac{u}{s}\right)\right)\left|\nabla_{g}^{\perp} u\right|_{g}^{2} d\|V\| \\
& +\frac{1}{s^{k}} \int_{B_{g}(\xi, s)} \gamma_{\varepsilon}\left(\frac{u}{s}\right)\left\langle\nabla_{g}^{S} h, u \nabla_{g} u\right\rangle_{g} d\|V\| \\
& +\frac{k c^{*}}{s^{k}} \int_{B_{g}(\xi, s)} h \gamma_{\varepsilon}\left(\frac{u}{s}\right) d\|V\| \\
& +\frac{1}{s^{k+1}} \int_{B_{g}(\xi, s)} \gamma_{\varepsilon}\left(\frac{u}{s}\right)\left\langle H_{g} h, u \nabla_{g} u\right\rangle_{g} d\|V\|,
\end{aligned}
$$

where $\gamma_{\varepsilon} \in C^{1}(\mathbb{R})$ is, such that, given $0<\varepsilon<1$,

$$
\gamma_{\varepsilon}(s):=\left\{\begin{array}{lll}
1, & \text { if } & s<\varepsilon \\
0, & \text { if } & s>1
\end{array}\right.
$$

and

$$
\gamma_{\varepsilon}^{\prime}(s)<0 \quad \text { if } \quad \varepsilon<s<1
$$

Then

$$
\begin{aligned}
\frac{d}{d s}\left(\frac{1}{s^{k}} \int_{B_{g}(\xi, s)} h \gamma_{\varepsilon}\left(\frac{u}{s}\right) d\|V\|\right) & \geq \frac{1}{s^{k+1}} \int_{B_{g}(\xi, s)} \gamma_{\varepsilon}\left(\frac{u}{s}\right)\left\langle H_{g} h+\nabla_{g}^{S} h, u \nabla_{g} u\right\rangle_{g} d\|V\| \\
& +\frac{c^{*} k}{s^{k}} \int_{B_{g}(\xi, s)} h \gamma_{\varepsilon}\left(\frac{u}{s}\right) d\|V\| \\
& \geq-\frac{1}{s^{k+1}} \int_{B_{g}(\xi, s)} \gamma_{\varepsilon}\left(\frac{u}{s}\right)\left|H_{g} h+\nabla_{g}^{S} h\right|_{g} u d\|V\| \\
& +\frac{c^{*} k}{s^{k}} \int_{B_{g}(\xi, s)} h \gamma_{\varepsilon}\left(\frac{u}{s}\right) d\|V\|,
\end{aligned}
$$


therefore

$$
\frac{d}{d s}\left(\frac{1}{s^{k}} \int_{B_{g}(\xi, s)} \gamma_{\varepsilon}\left(\frac{u}{s}\right) h d\|V\|\right) \geq-\frac{1}{s^{k}} \int_{B_{g}(\xi, s)} \gamma_{\varepsilon}\left(\frac{u}{s}\right)\left(\left|\nabla_{g}^{S} h\right|_{g}+h\left(\left|H_{g}\right|_{g}-c^{*} k\right)\right) d\|V\| .
$$

Now, let $\left\{\varepsilon_{j}\right\}_{j \in \mathbb{N}}$ a sequence of real numbers such that $\varepsilon \uparrow 1$ as $j \rightarrow \infty$, and $\left\{\gamma_{\varepsilon_{j}}\right\}_{j \in \mathbb{N}} \subset C^{1}(\mathbb{R})$ such that

$$
\gamma_{\varepsilon_{j}}(s):=\left\{\begin{array}{lll}
1, & \text { if } & s<\varepsilon_{j} \\
0, & \text { if } & s>1
\end{array}\right.
$$

and

$$
\gamma_{\varepsilon_{j}}^{\prime}(s)<0 \quad \text { if } \quad \varepsilon_{j}<s<1
$$

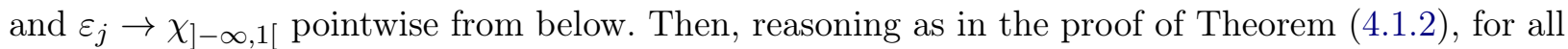
$j \in \mathbb{N}$, and for all $0<s<r_{0}$

$$
\frac{d}{d s}\left(\frac{1}{s^{k}} \int_{B_{g}(\xi, s)} \gamma_{\varepsilon_{j}}\left(\frac{u}{s}\right) h d\|V\|\right) \geq-\frac{1}{s^{k}} \int_{B_{g}(\xi, s)} \gamma_{\varepsilon_{j}}\left(\frac{u}{s}\right)\left(\left|\nabla_{g}^{S} h\right|_{g}+h\left(\left|H_{g}\right|_{g}-c^{*} k\right)\right) d\|V\| .
$$

Letting $j \rightarrow \infty$ we have in distributional sense in $s$,

$$
\frac{d}{d s}\left(\frac{1}{s^{k}} \int_{B_{g}(\xi, s)} h d\|V\|\right) \geq-\frac{1}{s^{k}} \int_{B_{g}(\xi, s)}\left(\left|\nabla_{g}^{S} h\right|_{g}+h\left(\left|H_{g}\right|_{g}-c^{*} k\right)\right) d\|V\| .
$$

Finally, integrating over $[\sigma, \rho] \subset] 0, r_{0}[$ we have

$$
\begin{aligned}
\int_{\sigma}^{\rho} \frac{d}{d s}\left(\frac{1}{s^{k}} \int_{B_{g}(\xi, s)} h d\|V\|\right) & =\frac{1}{\rho^{k}} \int_{B_{g}(\xi, \rho)} h d\|V\|-\frac{1}{\sigma^{k}} \int_{B_{g}(\xi, \sigma)} h d\|V\| \\
& \geq-\int_{\sigma}^{\rho}\left(\frac{1}{s^{k}} \int_{B_{g}(\xi, s)}\left(\left|\nabla_{g}^{S} h\right|_{g}+h\left(\left|H_{g}\right|_{g}-c^{*} k\right)\right) d\|V\|\right) d s .
\end{aligned}
$$

Therefore,

$$
\begin{aligned}
\frac{1}{\omega_{k} \sigma^{k}} \int_{B_{g}(\xi, \sigma)} h d\|V\| & \leq \frac{1}{\omega_{k} \rho^{k}} \int_{B_{g}(\xi, \rho)} h d\|V\| \\
& +\int_{\sigma}^{\rho} \frac{1}{s^{k}}\left(\int_{B_{g}(\xi, s)}\left|\nabla_{g}^{S} h\right|_{g}+h\left(\left|H_{g}\right|_{g}-c^{*} k\right) d\|V\|\right) d s .
\end{aligned}
$$

Lemma 5.2.2. Suppse $f$ and $g$ are bounded non-decreasing functions on $] 0, \infty[$, and

$$
1 \leq \frac{1}{\sigma^{k}} f(\sigma) \leq \frac{1}{\rho^{k}} f(\rho)+\int_{0}^{\rho} \frac{1}{s^{k}} g(s) d s,
$$

where $0<\sigma<\rho<\infty$. Then, there exists $\rho \in] 0, \rho_{0}[$ such that

$$
f(\rho) \leq \frac{1}{2} 5^{k} \rho_{0} g(\rho)
$$

where

$$
\rho_{0}=2\left(\lim _{\rho \rightarrow \infty} f(\rho)\right)^{\frac{1}{k}} .
$$


Proof. We argue by contradiction. Assume that for every $\rho \in] 0, \rho_{0}[$

$$
f(5 \rho)>\frac{1}{2} 5^{k} \rho_{0} g(\rho)
$$

then, by (5.11),

$$
\begin{aligned}
1 \leq \sup _{0<\sigma<\rho_{0}} \frac{1}{\sigma^{k}} f(\sigma) \leq & \frac{1}{\rho_{0}^{k}} f\left(\rho_{0}\right)+\int_{0}^{\rho_{0}} \frac{1}{s^{k}} g(s) d s \\
& <\frac{1}{\rho_{0}^{k}} f\left(\rho_{0}\right)+\frac{2}{5^{k} \rho_{0}} \int_{0}^{\rho_{0}} \frac{1}{s^{k}} f(5 s) d s \\
& =\frac{1}{\rho_{0}^{k}} f\left(\rho_{0}\right)+\frac{2}{5^{k} \rho_{0}} \int_{0}^{5 \rho_{0}} \frac{5^{k}}{s^{k}} f(s) \frac{d s}{5} \\
& =\frac{1}{\rho_{0}^{k}} f\left(\rho_{0}\right)+\frac{2}{5 \rho_{0}}\left(\int_{0}^{\rho_{0}} \frac{1}{s^{k}} f(s) d s+\int_{\rho_{0}}^{5 \rho_{0}} \frac{1}{s^{k}} f(s) d s\right) \\
& \leq \frac{1}{\rho_{0}^{k}} f\left(\rho_{0}\right)+\frac{2}{5} \sup _{0<s<\rho_{0}} \frac{1}{s^{k}} f(s)+\frac{2}{5 \rho_{0}} \lim _{s \rightarrow \infty} f(s) \int_{\rho_{0}}^{5 \rho_{0}} \frac{1}{s^{k}} d s \\
& \leq \frac{1}{\rho_{0}^{k}} \lim _{s \rightarrow \infty} f(s)+\frac{2}{5} \sup _{0<\sigma<\rho_{0}} \frac{1}{\sigma^{k}} f(\sigma) \\
& +\left(\frac{2}{5(1-n) \rho_{0}}\left(\left.\frac{1}{s^{k-1}}\right|_{\rho_{0}} ^{5 \rho_{0}}\right) \lim _{s \rightarrow \infty} f(s)\right) \\
\leq & \frac{1}{\rho_{0}^{k}} \lim _{s \rightarrow \infty} f(s)+\frac{2}{5(n-1)} \frac{1}{\rho_{0}^{k}} \lim _{s \rightarrow \infty} f(s)+\frac{2}{5} \sup _{0<\sigma<\rho_{0}} \frac{1}{\sigma^{k}} f(\sigma) \\
\leq & \left(1+\frac{2}{5}\right) \frac{1}{\rho_{0}^{k}} \lim _{s \rightarrow \infty} f(s)+\frac{1}{2} \sup _{0<\sigma<\rho_{0}} \frac{1}{\sigma^{k}} f(\sigma) \\
\leq & \left(1+\frac{1}{2}\right) \frac{1}{\rho_{0}^{k}} \lim _{s \rightarrow \infty} f(s)+\frac{1}{2} \sup _{0<\sigma<\rho_{0}} \frac{1}{\sigma^{k}} f(\sigma),
\end{aligned}
$$

then,

$$
\sup _{0<\sigma<\rho_{0}} \frac{1}{\sigma^{k}} f(\sigma)<3 \frac{1}{\rho_{0}^{k}} \lim _{s \rightarrow \infty} f(s) .
$$

Hence

$$
\sup _{0<\sigma<\rho_{0}} \frac{1}{\sigma^{k}} f(\sigma)<\frac{1}{2}\left(\sup _{0<\sigma<\rho_{0}} \frac{1}{\sigma^{k}} f(\sigma)+3 \frac{1}{\rho_{0}^{k}} \lim _{s \rightarrow \infty} f(s)\right)<3 \frac{1}{\rho_{0}^{k}} \lim _{s \rightarrow \infty} f(s)<4 \frac{1}{\rho_{0}^{k}} \lim _{s \rightarrow \infty} f(s),
$$

and then, by (5.14) we have,

$$
1 \leq \sup _{0<\sigma<\rho_{0}} \frac{1}{\sigma^{k}} f(\sigma)<\frac{1}{2}\left(\sup _{0<\sigma<\rho_{0}} \frac{1}{\sigma^{k}} f(\sigma)+3 \frac{1}{\rho_{0}^{k}} \lim _{s \rightarrow \infty} f(s)\right)<4 \frac{1}{\rho_{0}^{k}} \lim _{s \rightarrow \infty} f(s) .
$$

Finally, since

$$
\rho_{0}^{k}=2^{k} \lim _{s \rightarrow \infty} f(s)
$$

and dividing by 2 , we have

$$
\frac{1}{2} \leq \frac{1}{2} \sup _{0<\sigma<\rho_{0}} f(\sigma)<\frac{1}{2^{k-1}},
$$

which clearly is a contradiction since $k \geq 2$.

Now, we are ready to prove the Sobolev-type inequality, which we state bellow. 
Theorem 5.2.3 (Sobolev-Type Inequality for Intrinsic Varifolds). Let $\left(M^{n}, g\right)$ be a complete Riemannian manifold satisfying $(B G)$, let $V=\mathbf{v}(S, \theta)$ a rectifiable varifold satisfying $(A C)$. Suppose $h \in C_{0}^{1}(S)$ non negative, and $\theta \geq 1\|V\|$-a.e. in $S$. Then there exists $C:=C(k)>0$ such that

$$
\left(\int_{S} h^{\frac{k}{k-1}} d\|V\|\right)^{\frac{k-1}{k}} \leq C \int_{S}\left(\left|\nabla_{g}^{S} h\right|_{g}+h\left(\left|H_{g}\right|_{g}-c^{*} k\right)\right) d\|V\| .
$$

Proof. Notice that, for Lemma 5.2.1, for all $0<\sigma<\rho<r_{0}$

$$
\begin{aligned}
\frac{1}{\omega_{k} \sigma^{k}} \int_{B_{g}(\xi, \sigma)} h d\|V\| & \leq \frac{1}{\omega_{k} \rho^{k}} \int_{B_{g}(\xi, \rho)} h d\|V\| \\
& +\int_{\sigma}^{\rho} \frac{1}{s^{k}}\left(\int_{B_{g}(\xi, s)}\left|\nabla_{g}^{S} h\right|_{g}+h\left(\left|H_{g}\right|_{g}-c^{*} k\right) d\|V\|\right) d s,
\end{aligned}
$$

but, since by hypothesis $h \in C_{0}^{1}\left(B_{g}\left(\xi, r_{0}\right)\right)$ it remains valid for all $0<\sigma<\rho<\infty$, hence we can apply the Lemma 5.2.2 with the choices

$$
\begin{aligned}
f(\rho) & :=\frac{1}{\omega_{k}} \int_{B_{g}(\xi, \rho)} h d\|V\| \\
g(\rho) & :=\frac{1}{\omega_{k}} \int_{B_{g}(\xi, \rho)}\left(\left|\nabla_{g}^{S} h\right|_{g}+h\left(\left|H_{g}\right|_{g}-c^{*} k\right)\right) d\|V\|,
\end{aligned}
$$

provided that $\xi \in \operatorname{spt}\|V\|$ and $h(\xi) \geq 1$.

Thus for $\|V\|$-a.e $\xi \in\{x \in \operatorname{spt}\|V\|: h(x) \geq 1\}$ we have

$$
\rho<2\left(\frac{1}{\omega_{k}} \int_{M} h d\|V\|\right)^{\frac{1}{k}}
$$

and

$$
\int_{B_{g}(\xi, 5 \rho)} h d\|V\| \leq 5^{k}\left(\frac{1}{\omega_{k}} \int_{M} h d\|V\|\right)^{\frac{1}{k}} \int_{B_{g}(\xi, \rho)}\left(\left|\nabla_{g}^{S} h\right|_{g}+h\left(\left|H_{g}\right|_{g}-c^{*} k\right)\right) d\|V\| .
$$

Now, since $\{x \in \operatorname{spt} \| V \mid: h(x) \geq 1\} \subset \operatorname{spt}\|V\| \cap \operatorname{spt} h$ which is compact, in virtue of Vitali's five Lemma (cf. [Sim83] Theorem 3.3 pg. 11) we can select a collection of balls

$$
\left\{B_{g}\left(\xi_{i}, \rho_{i}\right)\right\}_{i \subset \Lambda},
$$

such that, for all $i \in \Lambda, \xi_{i} \subset\{x \in \operatorname{spt}\|V\|: h(x) \geq 1\}$ and, there exists $\Lambda^{\prime} \subset \Lambda$ such that for every $i \neq j, i, j \in \Lambda^{\prime}$ it holds $B_{g}\left(\xi_{i}, \rho_{i}\right) \cap B_{g}\left(\xi_{j}, \rho_{j}\right)=\emptyset$ and

$$
\{x \in \operatorname{spt}\|V\|: h(x) \geq 1\} \subset \bigcup_{j \in \Lambda^{\prime}} B_{g}\left(\xi_{i_{j}}, 5 \rho_{i_{j}}\right)=\bigcup_{i \in \Lambda} B_{g}\left(\xi_{i}, \rho_{i}\right) .
$$


Then, by (5.14)

$$
\begin{aligned}
\int_{\{h \geq 1\} \cap \text { spt }\|V\|} h d\|V\| & \leq \sum_{j \in \Lambda^{\prime}} \int_{B_{g}\left(\xi_{i_{j}}, 5 \rho_{i_{j}}\right)} h d\|V\| \\
& \leq \sum_{j \in \Lambda^{\prime}}\left(5^{k}\left(\frac{1}{\omega_{k}} \int_{M} h d\|V\|\right)^{\frac{1}{k}} \int_{B_{g}\left(\xi_{i_{j}}, \rho_{i_{j}}\right)}\left(\left|\nabla_{g}^{S} h\right|_{g}+h\left(|H|_{g}-c^{*} k\right)\right) d\|V\|\right) \\
& \leq 5^{k}\left(\frac{1}{\omega_{k}} \int_{M} h d\|V\|\right)^{\frac{1}{k}} \sum_{j \in \Lambda^{\prime}} \int_{B_{g}\left(\xi_{i_{j}}, \rho_{i_{j}}\right)}\left(\left|\nabla_{g}^{S} h\right|_{g}+h\left(|H|_{g}-c^{*} k\right)\right) d\|V\| \\
& \leq 5^{k}\left(\frac{1}{\omega_{k}} \int_{M} h d\|V\|\right)^{\frac{1}{k}} \int_{M}\left(\left|\nabla_{g}^{S} h\right|_{g}+h\left(|H|_{g}-c^{*} k\right)\right) d\|V\| .
\end{aligned}
$$

Let $\gamma_{\varepsilon} \in C^{1}(\mathbb{R})$ a non-decreasing function such that, for given $\varepsilon>0$,

$$
\gamma_{\varepsilon}(t):= \begin{cases}1, & \text { if } 0<\varepsilon<t \\ 0, & \text { if } t \leq 0,\end{cases}
$$

and consider, for $t_{0} \geq 0$ given,

$$
f(x):=\gamma_{\varepsilon}\left(h(x)-t_{0}\right),
$$

then, applying (5.15) with $f$ instead of $h$, and setting $S_{\alpha}:=\{x \in S: h(x)>\alpha\}$, we get,

$$
\begin{aligned}
\|V\|\left(S_{t_{0}+\varepsilon}\right) & =\int_{\left\{x \in M: h(x)-t_{0}>\varepsilon\right\}} h d\|V\| \\
& =\int_{\{x \in S: f(x) \geq 1\}} f d\|V\| \\
& \leq 5^{k}\left(\frac{1}{\omega_{k}} \int_{S} f d\|V\|\right)^{\frac{1}{k}} \int_{S}\left(\gamma_{\varepsilon}^{\prime}\left(h-t_{0}\right)\left|\nabla_{g}^{S} h\right|_{g}+f\left(\left|H_{g}\right|_{g}-c^{*} k\right)\right) d\|V\| \\
& \leq \frac{5^{k}}{\omega_{k}^{\frac{1}{k}}}\left(\|V\|\left(S_{t_{0}}\right)\right)^{\frac{1}{k}} \int_{S}\left(\gamma_{\varepsilon}^{\prime}\left(h-t_{0}\right)\left|\nabla_{g}^{S} h\right|_{g}+f\left(\left|H_{g}\right|_{g}-c^{*} k\right)\right) d\|V\|,
\end{aligned}
$$

now, multiplying by $\left(t_{0}+\varepsilon\right)^{\frac{1}{k-1}}$ we have,

$$
\begin{aligned}
\left(t_{0}+\varepsilon\right)^{\frac{1}{k-1}}\|V\|\left(S_{t_{0}+\varepsilon}\right) & \leq \frac{5^{k}}{\omega_{k}^{\frac{1}{k}}}\left(\left(t_{0}+\varepsilon\right)^{\frac{k}{k-1}}\|V\|\left(S_{t_{0}}\right)\right)^{\frac{1}{k}} \int_{S}\left(\gamma_{\varepsilon}^{\prime}\left(h-t_{0}\right)\left|\nabla_{g}^{S} h\right|_{g}+f\left(|H|_{g}-c^{*} k\right)\right) d\|V\| \\
& =\frac{5^{k}}{\omega_{k}^{\frac{1}{k}}}\left(\int_{S_{t_{0}}}\left(t_{0}+\varepsilon\right)^{\frac{k}{k-1}} d\|V\|\right)^{\frac{1}{k}} \int_{S}\left(\gamma_{\varepsilon}^{\prime}\left(h-t_{0}\right)\left|\nabla_{g}^{S} h\right|_{g}+f\left(|H|_{g}-c^{*} k\right)\right) d\|V\| \\
& \leq \frac{5^{k}}{\omega_{k}^{\frac{1}{k}}}\left(\int_{S_{t_{0}}}(h+\varepsilon)^{\frac{k}{k-1}} d\|V\|\right)^{\frac{1}{k}}\left(\int_{S}-\frac{\partial}{\partial t_{0}} \gamma_{\varepsilon}^{\prime}\left(h-t_{0}\right)\left|\nabla_{g}^{S} h\right|_{g} d\|V\|+\int_{S} f\left(|H|_{g}-c^{*} k\right) d\|V\|\right) \\
& \leq \frac{5^{k}}{\omega_{k}^{\frac{1}{k}}}\left(\int_{S}(h+\varepsilon)^{\frac{k}{k-1}} d\|V\|\right)^{\frac{1}{k}}\left(\int_{S}-\frac{\partial}{\partial t_{0}} \gamma_{\varepsilon}^{\prime}\left(h-t_{0}\right)\left|\nabla_{g}^{S} h\right|_{g} d\|V\|+\int_{S} f\left(|H|_{g}-c^{*} k\right) d\|V\|\right) .
\end{aligned}
$$

Integrating the above inequality on $t_{0}$ in the interval $] 0, \infty[$, we have

$$
\begin{aligned}
& \int_{0}^{\infty}\left(t_{0}+\varepsilon\right)^{\frac{1}{k-1}}\|V\|\left(S_{t_{0}+\varepsilon}\right) d t_{0} \leq \\
& \quad \frac{5^{k}}{\omega_{k}^{\frac{1}{k}}}\left(\int_{S}(h+\varepsilon)^{\frac{k}{k-1}} d\|V\|\right)^{\frac{1}{k}} \int_{0}^{\infty}\left(\int_{S}-\frac{\partial}{\partial t_{0}} \gamma_{\varepsilon}^{\prime}\left(h-t_{0}\right)\left|\nabla_{g}^{S} h\right|_{g} d\|V\|+\int_{S} f\left(|H|_{g}-c^{*} k\right) d\|V\|\right) d t_{0} .
\end{aligned}
$$


First, notice that the left hand side, by the Lemma 4.2.3, is equal to,

$\int_{0}^{\infty}\left(t_{0}+\varepsilon\right)^{\frac{1}{k-1}}\|V\|\left(S_{t_{0}+\varepsilon}\right) d t_{0}=\frac{k-1}{k} \int_{S_{0}}(h-\varepsilon)^{\frac{k}{k-1}} d\|V\|=\frac{k-1}{k} \int_{\{x \in S: h(x)>\varepsilon\}}(h-\varepsilon)^{\frac{k}{k-1}} d\|V\|$.

on the other hand, we can estimate the right hand side as follows, first by Fubini's Theorem

$$
\begin{aligned}
\int_{0}^{\infty}\left(\int_{S}-\frac{\partial}{\partial t_{0}} \gamma_{\varepsilon}^{\prime}\left(h(x)-t_{0}\right)\left|\nabla_{g}^{S} h\right|_{g} d\|V\|\right) d t_{0} & =\int_{S}\left|\nabla_{g}^{S} h\right|_{g}\left(-\frac{d}{d t} \int_{0}^{\infty} \gamma_{\varepsilon}\left(h(x)-t_{0}\right) d t_{0}\right) d\|V\| \\
& =\int_{S}\left|\nabla_{g}^{S} h\right|_{g}\left(\gamma_{\varepsilon}(h(x))\right) d\|V\| \\
& \leq \int_{S}\left|\nabla_{g}^{S} h(x)\right| d\|V\| .
\end{aligned}
$$

And, again by Fubini's Theorem,

$$
\begin{aligned}
\int_{0}^{\infty}\left(\int_{S} f(x)\left(\left|H_{g}\right|_{g}-c^{*} k\right) d\|V\|(x)\right) d t & =\int_{S}\left(\left|H_{g}\right|_{g}-c^{*} k\right)\left(\int_{0}^{\infty} \gamma_{\varepsilon}\left(h(x)-t_{0}\right) d t_{0}\right) d\|V\| \\
& =\int_{S}\left(\left|H_{g}\right|_{g}-c^{*} k\right)\left(\int_{h(x)}^{-\infty}-\gamma_{\varepsilon}(w) d w\right) d\|V\| \\
& \leq \int_{S} h\left(\left|H_{g}\right|_{g}-c^{*} k\right) d\|V\| .
\end{aligned}
$$

Therefore

$$
\frac{k-1}{k} \int_{M_{\varepsilon}}(h-\varepsilon)^{\frac{k}{k-1}} d\|V\| \leq \frac{5^{k}}{\omega_{k}^{\frac{1}{k}}}\left(\int_{M}(h+\varepsilon)^{\frac{k}{k-1}} d\|V\|\right)^{\frac{1}{k}} \int_{M}\left(\left|\nabla_{g}^{S} h\right|_{g}+h\left(\left|H_{g}\right|_{g}-c^{*} k\right)\right) d\|V\|,
$$

then, letting $\varepsilon \rightarrow 0$ we obtain

$$
\left(\int_{S} h^{\frac{k}{k-1}} d\|V\|\right)^{\frac{k-1}{k}} \leq C \int_{S}\left(\left|\nabla_{g}^{S} h\right|_{g}+h\left(\left|H_{g}\right|_{g}-c^{*} k\right)\right) d\|V\|
$$

where

$$
C:=C(k):=\frac{k 5^{k}}{(k-1) \omega_{k}^{\frac{1}{k}}}
$$




\section{Bibliography}

[AFP00] Luigi Ambrosio, Nicola Fusco e Diego Pallara. Functions of bounded variation and free discontinuity problems. Oxford Mathematical Monographs. The Clarendon Press, Oxford University Press, New York, 2000. 25

[AK00] Luigi Ambrosio e Bernd Kirchheim. Rectifiable sets in metric and Banach spaces. Math. Ann., 318(3):527-555, 2000. 4

[All72] William K. Allard. On the first variation of a varifold. Ann. of Math. (2), 95:417-491, 1972. $2,3,5,25,26$

[Alm68] F. J. Almgren, Jr. Existence and regularity almost everywhere of solutions to elliptic variational problems among surfaces of varying topological type and singularity structure. Ann. of Math. (2), 87:321-391, 1968.

[BDGM69a] E. Bombieri, E. De Giorgi e M. Miranda. Una maggiorazione a priori relativa alle ipersuperfici minimali non parametriche. Arch. Rational Mech. Anal., 32:255-267, 1969. 1

[BDGM69b] E. Bombieri, E. De Giorgi e M. Miranda. Una maggiorazione a priori relativa alle ipersuperfici minimali non parametriche. Arch. Rational Mech. Anal., 32:255-267, 1969. 1

[DG61] Ennio De Giorgi. Frontiere orientate di misura minima. Seminario di Matematica della Scuola Normale Superiore di Pisa, 1960-61. Editrice Tecnico Scientifica, Pisa, 1961.

[Eva10] Lawrence C. Evans. Partial differential equations, volume 19 of Graduate Studies in Mathematics. American Mathematical Society, Providence, RI, second edição, 2010. 1

[Fed69] Herbert Federer. Geometric measure theory. Die Grundlehren der mathematischen Wissenschaften, Band 153. Springer-Verlag New York Inc., New York, 1969. 9, 12, 13

[FF60] Herbert Federer e Wendell H. Fleming. Normal and integral currents. Ann. of Math. (2), 72:458-520, 1960. 1, 2

[GMS15] Nicola Gigli, Andrea Mondino e Giuseppe Savaré. Convergence of pointed noncompact metric measure spaces and stability of Ricci curvature bounds and heat flows. Proc. Lond. Math. Soc. (3), 111(5):1071-1129, 2015.

[GT01] David Gilbarg e Neil S. Trudinger. Elliptic partial differential equations of second order. Classics in Mathematics. Springer-Verlag, Berlin, 2001. Reprint of the 1998 edition.

[Heb99] Emmanuel Hebey. Nonlinear analysis on manifolds: Sobolev spaces and inequalities, volume 5 of Courant Lecture Notes in Mathematics. New York University, Courant Institute of Mathematical Sciences, New York; American Mathematical Society, Providence, RI, 1999. 
[HS74] David Hoffman e Joel Spruck. Sobolev and isoperimetric inequalities for Riemannian submanifolds. Comm. Pure Appl. Math., 27:715-727, 1974. 2, 14, 32

[Jos17] Jürgen Jost. Riemannian geometry and geometric analysis. Universitext. Springer, Cham, seventh edição, 2017. 20, 21

[Lel12] Camillo De Lellis. Allard's interior regularity theorem: an invitation to stationary varifolds. http://www.math.uzh.ch/fileadmin/user/delellis/publikation/allard_35.pdf, 2012. 25

[Mag12] Francesco Maggi. Sets of finite perimeter and geometric variational problems, volume 135 of Cambridge Studies in Advanced Mathematics. Cambridge University Press, Cambridge, 2012. An introduction to geometric measure theory. 7, 8, 12

[Mat95] Pertti Mattila. Geometry of sets and measures in Euclidean spaces, volume 44 of Cambridge Studies in Advanced Mathematics. Cambridge University Press, Cambridge, 1995. Fractals and rectifiability. 42

[Mir67] Mario Miranda. Diseguaglianze di Sobolev sulle ipersuperfici minimali. Rend. Sem. Mat. Univ. Padova, 38:69-79, 1967. 1

[MN19] Andrea Mondino e Aaron Naber. Structure theory of metric measure spaces with lower Ricci curvature bounds. J. Eur. Math. Soc. (JEMS), 21(6):1809-1854, 2019.

[MS73] J. H. Michael e L. M. Simon. Sobolev and mean-value inequalities on generalized submanifolds of $R^{n}$. Comm. Pure Appl. Math., 26:361-379, 1973. 2

[Nar09] Stefano Nardulli. The isoperimetric profile of a smooth Riemannian manifold for small volumes. Ann. Global Anal. Geom., 36(2):111-131, 2009.

[Nar14] Stefano Nardulli. The isoperimetric profile of a noncompact Riemannian manifold for small volumes. Calc. Var. Partial Differential Equations, 49(1-2):173-195, 2014.

[Nar18] Stefano Nardulli. Regularity of isoperimetric regions that are close to a smooth manifold. Bull. Braz. Math. Soc. (N.S.), 49(2):199-260, 2018. 2

[Nas54] John Nash. $C^{1}$ isometric imbeddings. Ann. of Math. (2), 60:383-396, 1954. 2

[NO16] S. Nardulli e L. E. Osorio Acevedo. Sharp isoperimetric inequalities for small volumes in complete noncompact Riemannian manifolds of bounded geometry involving the scalar curvature. ArXiv e-prints, Novembro 2016.

[NOA18] Stefano Nardulli e Luis Eduardo Osorio Acevedo. Sharp Isoperimetric Inequalities for Small Volumes in Complete Noncompact Riemannian Manifolds of Bounded Geometry Involving the Scalar Curvature. International Mathematics Research Notices, 062018. rny131. 2,25

[Pet06] Peter Petersen. Riemannian geometry, volume 171 of Graduate Texts in Mathematics. Springer, New York, second edição, 2006. 17

[Rei60] E. R. Reifenberg. Solution of the Plateau Problem for $m$-dimensional surfaces of varying topological type. Acta Math., 104:1-92, 1960.

[Rei64] E. R. Reifenberg. An epiperimetric inequality related to the analyticity of minimal surfaces. Ann. of Math. (2), 80:1-14, 1964.

[Rud87] Walter Rudin. Real and complex analysis. McGraw-Hill Book Co., New York, third edição, 1987. 29 
[Sim83] Leon Simon. Lectures on geometric measure theory, volume 3 of Proceedings of the Centre for Mathematical Analysis, Australian National University. Australian National University, Centre for Mathematical Analysis, Canberra, 1983. 2, 3, 4, 5, 7, 9, $10,13,25,32,38,54$

[War83] Frank W. Warner. Foundations of differentiable manifolds and Lie groups, volume 94 of Graduate Texts in Mathematics. Springer-Verlag, New York-Berlin, 1983. Corrected reprint of the 1971 edition. 13 